\title{
PARKINSON'S DISEASE
}

\author{
by
}

Alejandro Soto-Avellaneda

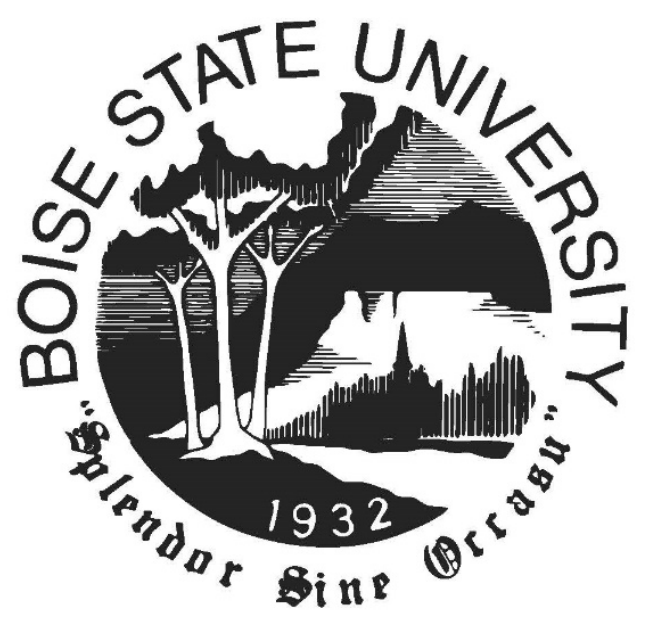

\author{
A dissertation \\ submitted in partial fulfillment \\ of the requirements for the degree of \\ Doctor of Philosophy in Biomolecular Sciences
}

Boise State University

August 2021 
(C) 2021

Alejandro Soto-Avellaneda

\section{ALL RIGHTS RESERVED}




\title{
BOISE STATE UNIVERSITY GRADUATE COLLEGE
}

\section{DEFENSE COMMITTEE AND FINAL READING APPROVALS}

\author{
of the dissertation submitted by
}

\begin{abstract}
Alejandro Soto-Avellaneda
Dissertation Title: Autophagy Regulation by Lipid Factors with Implications for Parkinson's Disease

Date of Final Oral Examination: $\quad 29$ July 2021

The following individuals read and discussed the dissertation submitted by student Alejandro Soto-Avellaneda, and they evaluated the student's presentation and response to questions during the final oral examination. They found that the student passed the final oral examination.

Brad E Morrison, Ph.D. Chair, Supervisory Committee

Daniel Fologea, Ph.D. Member, Supervisory Committee

Eric Hayden, Ph.D. Member, Supervisory Committee

The final reading approval of the dissertation was granted by Brad E Morrison, Ph.D., Chair of the Supervisory Committee. The dissertation was approved by the Graduate College.
\end{abstract}




\section{DEDICATION}

I would like to dedicate this work to my mother, Carmen Avellaneda, and my

wife, Katelin Soto, whose undying love, support, and encouragement were instrumental in getting to this far. ¡Sí se puede! 


\section{ACKNOWLEDGMENTS}

I would first like to thank Dr. Brad Morrison, my advisor and mentor. Thank you for giving me the opportunity to follow my dream and for always being there to guide me. I would also like to acknowledge the members of my supervisory committee, Dr. Eric Hayden, and Dr. Daniel Fologea. You have been amazing teachers, and I appreciate all your advice and everything you have taught me. I would also like to recognize Dr. Eric Ortlund and the staff at the Emory Integrated Metabolomics and Lipidomics Core whose lipidomic analysis made this work possible. Finally, I would like to acknowledge and thank all the young researchers who I have had the opportunity to work with in the Morrison lab. You have all contributed to this work in one way or another. I appreciate your help, and I hope you all go on to do great things. 


\begin{abstract}
Parkinson's disease is the second most common neurodegenerative disorder. It is characterized by the death of dopaminergic neurons in the substantia nigra and a series of debilitating motor symptoms. Macroautophagy (hereafter referred to as autophagy) is a cellular process by which cells degrade proteins, lipids, organelles or dysfunctional components. Autophagy is thought to play an important role in Parkinson's disease, because it is the only cellular process known to remove large protein aggregates, such as those seen in Parkinson's disease pathology. Historically, a large body of work has focused on reporting on protein effectors of autophagy, and regulation of autophagy but lipophilic molecules has garnered less attention. This dissertation focuses on the regulatory contributions of lipid molecules to autophagy in addition to describing the identification and lead discovery of autophagy-regulating lipid factors using an endogenous lipid chaperone protein, known as Fatty Acid Binding Protein 5, as a 'bait' molecule.
\end{abstract}




\section{TABLE OF CONTENTS}

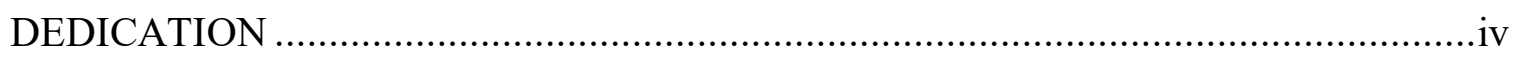

ACKNOWLEDGMENTS ............................................................................

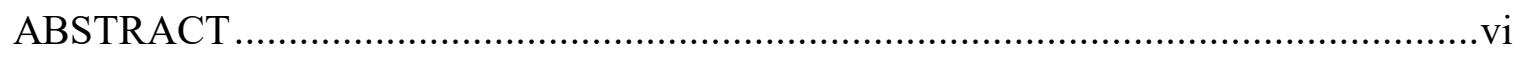

TABLE OF CONTENTS..............................................................................

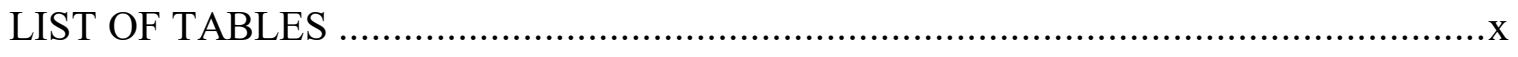

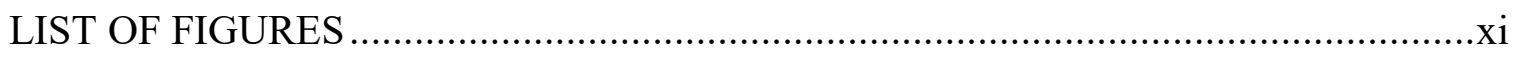

LIST OF ABBREVIATIONS .....................................................................xviii

CHAPTER ONE: A REVIEW OF SIGNALING AND OTHER ROLES OF LIPIDS IN

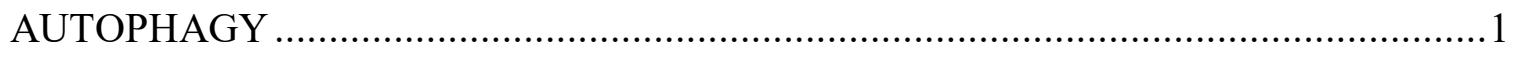

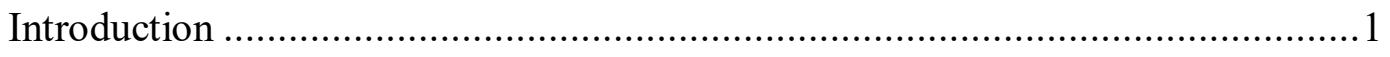

Lipid Signaling Directs Autophagy .................................................. 4

Impaired Autophagy in Human Disease ..................................................

Phospholipids, Sphingolipids, and mTOR Signaling …............................. 9

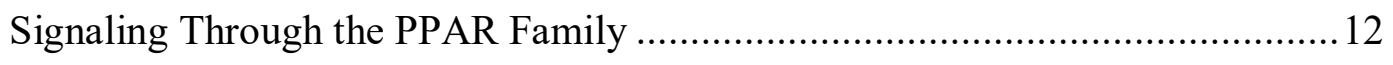

Lipid Metabolism and Autophagy ….................................................. 15

Free Fatty Acids and Cholesterol...................................................... 16

Conclusion and Future Perspectives ................................................... 20

CHAPTER TWO: LIPIDOMIC ANALYSIS OF FABP5-BOUND AUTOPHAGY

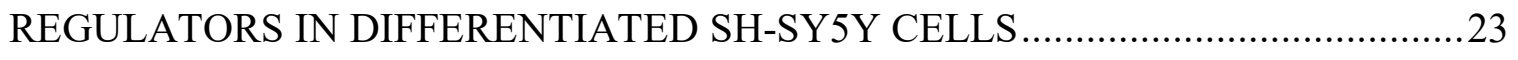

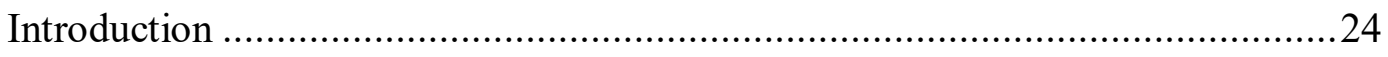


Autophagy and Parkinson's Disease .............................................. 24

Regulation of Autophagy by Lipids .......................................... 25

FABP5 is an Intracellular Lipid Chaperone ..................................... 25

Dopaminergic SH-SY5Y cells............................................... 26

Materials And Methods ....................................................................... 26

Cell Culture ................................................................................ 26

Lipid Treatment of Cells ..................................................... 27

Detergent-free Immunoprecipitation............................................ 28

Western Blotting ...................................................................... 28

Lentiviral Production and Transduction......................................... 29

LC3B-GFP-Mcherry Puncta Assessment......................................... 30

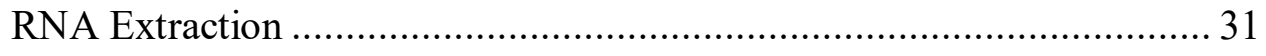

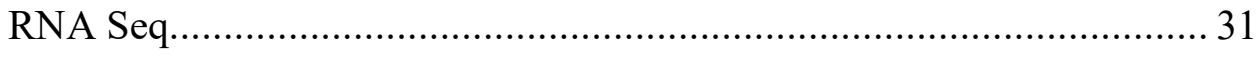

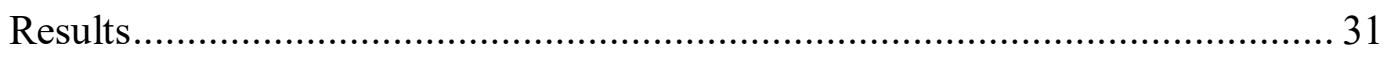

FABP5 is Expressed by Dopaminergic Neurons in the Substantia Nigra 31

FABP5 Regulates Autophagy ................................................. 32

5OE, AA, HSA, SA and PA Interact with FABP5 …......................... 34

Treatment with 5OE, HSA or SA Enhances mTORC1 Pathway Signaling

5OE, HSA and SA Do Not Induce Cell Death .................................. 39

Transcriptomic Alteration of the PPAR Pathway by HSA .................. 40

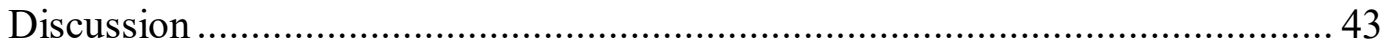

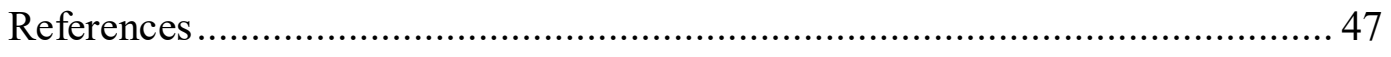

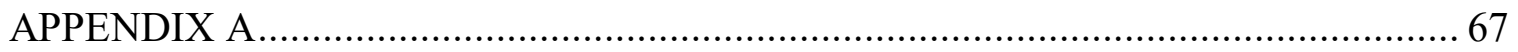


APPENDIX B

112 


\section{LIST OF TABLES}

Table 1.1 A Summary of Discussed Lipids Involved in Autophagy......................21

Table A1 Cell lineage tracing results for vascular endothelial cadherin and Nestin 77

Table B1. Examples of repurposed drugs............................................ 115 


\section{LIST OF FIGURES}

Figure 1.1 Autophagy mechanism. Autophagy is a cellular mechanism by which metabolites, organelles, proteins, and protein aggregates are enveloped by a vesicular membrane to form an autophagosome. The autophagosome is trafficked to a lysosome where fusion occurs, and lysosomal degradative enzymes break down the cargo.

Figure 1.2 PI3K-mTOR autophagy pathway utilizes PIP3 lipid signaling. PI3K converts the lipid PIP2 to PIP3. PIP3 mediates the phosphorylation of PDK1 causing the activation of AKT. AKT inhibits the activation of the TSC1/2 complex by phosphorylating TSC2. The inhibition of the TSC1/2 complex results in inhibition of Rheb GTPase which in turn activates mTORC1. MTORC1 inhibits the activation of the ULK activation complex leading to an inhibition of autophagy. Inversely, phosphatase activity of PTEN converts PIP3 to PIP2 which suppresses the activation of PDK1 and downstream AKT. Inhibited AKT cannot suppress the TSC1/2 complex allowing Rheb GTPase to remain active. An active Rheb results in inhibition of mTORC1 and an activation of the ULK1 complex...........7

Figure 1.3 Overview of PPAR signaling and mechanism for PPAR $\alpha$-mediated autophagy activation in innate immune system. Initially, lipid molecule enters the cell and is quickly bound by a fatty acid binding protein. The fatty acid binding protein transports the lipid to a PPAR which in turn activates a corresponding RXR. The PPAR-RXR complex crosses into the nucleus and facilitate expression of required genes. In the case of tuberculosis infection, PPAR $\alpha$ upregulates the expression of TFEB which, in turn, drives the expression of autophagy related genes, LAMP3 and RAB7 thus stimulating autophagy.

Figure 1.4 PPAR $\gamma$ mediated activation of autophagy. PPAR $\gamma$ promotes the expression of PTEN. High amounts of PTEN lead to lower concentrations of PI(3,4,5)P3. Less PI(3,4,5)P3 inhibits the activation of PDK1 and ultimately results in inhibition of $\mathrm{mTORC} 1$ which causes an activation of autophagy.

Figure 1.5 Overview of regulation of autophagy by free fatty acids. Palmitic acid stimulates autophagy by activating JNK and has been shown to interact with PKC isoforms which have been implicated in autophagy regulation. Myristic acid has been shown to upregulate autophagy by producing C-14 
ceramide. DHA has been implicated in the P53-AMPK-mTOR pathway resulting in activation of autophagy.

Figure 2.1. FABP5 is expressed by nigral dopaminergic neurons. In situ hybridization for Tyrosine hydroxylase (A) and Fabp 5 (B) mRNA expression [Mouse Brain Atlas, Allen Brain Institute]. Immunohistochemistry was performed for FABP5 (C) and Tyrosine Hydroxylase (D) in adult mouse brain substantia nigra with nuclei labeled using DAPI and merged image shown (E). Scale bar $=400 \mu \mathrm{m}$.

Figure 2.2. FABP5 regulates autophagy. (A) Undifferentiated or RA-differentiated SH-SY5Y cells were transduced with lentivirus containing scrambled (Cntrl) or FABP5 shRNA constructs and LC3B-IIexpression evaluated by western blot and (B) autophagic flux determined. (C) Representative images for mCherry-gfp-LC3B that was transduced by lentivirus into RAdifferentiated control or FABP5 knockdown as described in A. and then autophagosome (yellow) and lysosomes (red) quantified (D). *p $<0.05$, error bars $=$ SEM.

Figure 2.3. FABP5 does not affect SH-SY5Y differentiation by RA. SH-SY5Y cells were transduced with lentivirus containing a scrambled (control) shRNA, FABP5 shRNA or an FABP5 overexpression (OVER) construct. Cells were selected for transgene expression using puromycin and treated with all-trans RA $(10 \mu \mathrm{M})$ for the indicated times and Tyrosine Hydroxylase $(\mathrm{TH})$ and Dopamine receptor $\mathrm{D}_{2}$ (DRD2) protein levels were assessed by western blot. 3 biological replicates are shown.

Figure 2.4. FABP5 lipidomic interactome.(A) FABP5 was successfully immunoprecipitated (IP) with a detergent-free method using lysate obtained from RA-differentiated SH-SY5Y cells that stably express a recombinant FABP5-V5. Immunoprecipitations (V5 epitope tag-specific or antibody isotype control) were performed for $1 \mathrm{hr}$ or overnight $(\mathrm{O} / \mathrm{N})$ and western blotting done using an FABP5 antibody. (B) Samples obtained by $1 \mathrm{hr}$ FABP5-V5 IP (V5) or control antibody IP (Control) from 3 independent biological replicates were processed for lipid extraction and analyzed by QTRAP 5500 mass spectrometer via Exion LC AC autosampler. Six lipids were identified to have significant interaction with FABP5. .35

Figure 2.5. Effect of candidate lipids on LC3B-II autophagic flux. (A,C,E,G,I) Increasing amounts of lipid or vehicle (ethanol) were added to RAdifferentiated SH-SY5Y cells for $4 \mathrm{~h}$ in the presence or absence of bafilomycin A1 (Baf A1) and western blotting performed and autophagic flux was determined (B,D,F,H,J) from 3 biological replicates. ${ }^{*} \mathrm{p}<0.05$, error bars $=$ SEM 
Figure 2.6. Effect of candidate lipids on LC3B puncta autophagic flux. Lentivirus was used to transduce RA-differentiated SH-SY5Y cells with mCherry-gfpLC3B. Seven days later, cells were treated with ethanol (vehicle) or lipid at the highest two does for $4 \mathrm{~h}$. Representative images (A) are shown for the highest lipid dose and autophagosomes (yellow) and lysosomes (red) were then quantified (B). ${ }^{*} \mathrm{p}<0.05$, error bars $=$ SEM.

Figure 2.7. mTOR pathway activation by 5OE, HSA, SA, and PA. SH-SY5Y cells were differentiated with RA and then subjected to ethanol (vehicle), 5OE, HSA, SA or PA for the indicated concentrations and times in freshly prepared culture media. Western blotting (A, B) was then performed for pS6 (Ser240/244) following treatment at the indicated times. Densitometry was assessed for $\mathrm{pS6/B-Actin} \mathrm{for} \mathrm{each} \mathrm{condition} \mathrm{and} \mathrm{time} \mathrm{point}$ normalized to vehicle for each time point $(C, D) . * p<0.05$, error bars $=$ SEM versus vehicle group at each time point

Figure 2.8. 5OE, HSA, SA and PA do not induce cell death. RA-differentiated SHSY5Y cells were treated with ethanol (vehicle), 5OE, HSA, SA or PA as shown in normal growth media. After $24 \mathrm{~h}$, cell death was measured by (A, C) $\mathrm{LDH}$ released into the culture media as well as y the $(\mathrm{B}, \mathrm{D})$ resazurin assay. $* \mathrm{p}<0.05$, error bars $=\mathrm{SEM}$.

Figure 2.9. RNA Seq analysis of lipid treatment.KEGG annotated pathways for RAdifferentiated SH-SY5Y cells treated with (A) $4 \mu \mathrm{M}$ 5OE, (C) $400 \mu \mathrm{M}$ HSA and (E) $400 \mu \mathrm{M}$ SA for $6 \mathrm{~h}$. (B, D, F) Reactome pathways are also shown for each treatment. *significance versus vehicle treatment group; 3 biological replicates. Volcano plots for (G) $4 \mu \mathrm{M}$ 5OE, (F) $400 \mu \mathrm{M}$ HSA and $(\mathrm{G}) 400 \mu \mathrm{M}$ SA are shown (red dots = up regulated genes, green dots = down regulated genes).

Figure A1 Transgenic mouse cell lineage tracing strategy. (A). Adult mice (4-5 months of age) possessing a transgenic cassette containing either vascular endothelial cadherin (VEcad)- or Nestin-promoter-driven.CreER ${ }^{\mathrm{T} 2}$ recombinase are fed tamoxifen (TAM) citrate-laden chow to acti- vate loxP site-directed excision of a second transgene (Ai9 line) possessing a translation stop codon sequence. Removal of this stop codon results in CAG promoter-driven reporter expression of tdTomato. Note that postTAM tdTomato expression is solely controlled by the CAG promoter and Gt(RO- SA)26Sor chromosomal locus (tdTomato reporter locus). (B) Permanent activation of tdTomato expression in VEcad or Nestinexpressing cells was achieved by feeding adult mice tamoxifen for 1.5 months. Mice were sacri- ficed at the indicated times, fixed and histological assessment was performed. .76

Figure A2 Vascular endothelial-cadherin cell fate mapping in the central nervous system. Adult mice possessing VEcad-CreER ${ }^{T 2} /$ Rosa-flox-STOP- 
tdTomato were fed tamoxifen-containing chow for 1.5 months and mice sacrificed at the indicated times following initiation of treatment.

Representative channel merged images from fixed and Hoechst labeled tissues. Nuclei are labeled by Hoechst (blue) and cell lineage tracing is indicated by tdTomato (red). The areas shown in micrographs are indicated by high- lighted regions (red boxes) in diagrams to the right where the granule cell layer (GCL), glomerular layer (GL), lateral ventricle (LV) and fourth ventricle (4V) are shown. Arrowheads highlight labeled cells and cellular appendages (white scale bars: $400 \mu \mathrm{m}$ ).

Figure A3 Cerebellar granule neurons and hair follicle stem cells express vascular endothelial-cadherin fate mapping reporter. Representative confocal microscopy images from adult VEcad-CreERT2/Rosa-flox-STOPtdTomato transgenic mouse cerebellum $(\mathrm{A})$ and skin $(\mathrm{B}, \mathrm{C})$ at three months following the start of tamoxifen treatment. Immunohis- tochemical labeling for (A) NeuN (green) or (B, C) K15 (green) with tdTo- mato (red) and nucleus (Hoechst; blue) are shown at two magnifications to demonstrate the rare occurrence of K15-tdTomato colocalization. Images captured using a Zeiss Laser Scanning Confocal Microscope Meta 510 sys- tem. Orthogonal projections provided in the supplementary data indicate cells exhibiting (\#) red/green colocalization. Asterisks (*) signify cells with- out co-localization, hair shafts (HS) are also shown. 80

Figure A4 Vascular endothelial-cadherin cell fate mapping in the periphery. 4-5Month-old VEcad-CreERT2/Rosa-flox-STOP-tdTomato mice were provided tamoxifen chow for 1.5 months and sacrificed at the times shown. Images were obtained from fixed and Hoechst stained tissues. Cell lineage tracing is evidenced by tdTomato (red) and nuclei are indicated by Hoechst (blue) labeling. Arrowheads highlight labeled cells (white scale bar $=400 \mu \mathrm{m})$. Red boxes displayed in diagrams indicate region shown in the associated micrographs. Asterisk (*) indicates tdTomato+ muscle fibers underlying skin; Intestinal lumen (L) and tdTomato+ crypt cells (arrowheads) are labeled. Renal tissue diagram indicates the location of the renal pelvis (R.P.).

Figure A5 Vascular endothelial-cadherin fate mapping labels pancreatic $\beta$ cells.Immunohistochemistry was performed for pancreatic insulin (A, B) and tdTomato reporter expression at three months post-tamoxifen treatment in adult VEcad-CreER $R^{T 2} /$ Rosa-flox-STOP-tdTomato transgenic mice. Representative images were obtained by confocal microscopy for insulin (A, B; green), glucagon (C; green), tdTomato (red) and the nucleus (Hoechst; blue). Two magnifications of separate fields are shown to demonstrate the rare instances of Insulin-tdTomato colocalization. A Zeiss Laser Scanning Confocal Microscope Meta 510 system was used to capture and analyze the images shown. Confocal orthogonal projections 
presented in the supplemental data section were utilized to confirm cells with (\#) or without (*) green/red co-localization ......................................84

Figure A6 Nestin cell fate mapping in the central nervous system. Transgenic NesCreER ${ }^{T 2} /$ Rosa-flox-STOP-tdTomato mice at 4-5 months of age were fed tamoxifen-lad- en chow for 1.5 months and then sacrificed at thetimes shown post treatment initiation. Channel merged images were then acquired from fixed and Hoechst stained tissues. Nuclei are labeled by Hoechst (blue) and cell lineage tracing is indicated by tdTomato (red). Images shown were obtained from the regions indicated by the red box in the as- sociated diagram to the right where the granule cell layer (GCL), glomerular layer (GL), lateral ventricle (LV) and fourth ventricle (4V) are shown. Arrow- heads highlight labeled cells and cellular append- ages; \# indicates autofluorescence from wrinkled tissue region (white scale bars: $400 \mu \mathrm{m}$; green scale bars: $1000 \mu \mathrm{m})$.

Figure A7 Nestin cell fate mapping in the periphery. Transgenic Nes-CreER ${ }^{T 2} / \mathrm{Rosa}$ flox-STOP-tdTomato mice at 4-5 months were fed tamoxifen-laden chow and tissue har- vested at the indicated times following treatment initiation. The representative merged channel images were acquired from tissues that were fixed and Hoechst labeled. Cell lineage trac- ing is evidenced by tdTomato (red) and nuclei are indicated by Hoechst (blue) labeling. Note in hair follicles at 1.5 months after tamoxifen addition, the observed fluorescent signal is only found in hair shafts (no labeled cells). Labeled hair folli- cle cells observed at 6 months post-tamoxifen were confirmed K15-negative. Red boxes displayed in diagrams on the right indicate region shown in the associated micrographs. Asterisk $(*)$ indicates tdTomato ${ }^{+}$muscle fibers underlying skin. Arrow- heads highlight labeled cells and the ileal lumen (L) is indicat- ed [white scale bar: $400 \mu \mathrm{m}$ ].......89

Figure AS1. Prototypical arterial endothelila cell expression of dTomato following VEcadherin cell-lineage tracing in real tissue. Renal tissue was harvested from VEcad-CreER ${ }^{T 2} /$ Rosa-flox-STOP-tdTomato transgenic mice 1.5 months post-tamoxifen treatment initiation. Tissue samples were fixed, cryosectioned $(15 \mu \mathrm{m})$ onto slides, stained with Hoechst dye (nuclear label) and then mounted with coverslips. The displayed imagewas captured by an EVOS M7000 conventional fluorescence microscopy system. The merged image shows tdTomato (red) and Hoechst (blue) channels. Arrowhead indicates tdTomato+ endothelial cells of the internal elastic lamina of a renal glomerulus arteriole. Asterisks $(*)$ show tdTomato + convoluted tubules also present in this micrograph. .99

Figure AS2. Confocal microscopy orthogonal analysis of VE-cadherin-traced cerebellargranule neurons and hair follicle cells presented in Figure A3. (A) NeuN (green) /tdTomato (red) co-labeling (arrowheads) by IHC confirmed for cerebellar granule neurons through orthogonal 
reconstruction from confocal image stacks. $(\mathrm{B}, \mathrm{C})$ Rare hair follicular cells exhibit K15 (green)/tdTomato (red) co-localization (arrowheads). (D) The vast majority of tdTomato hair follicle cells do not exhibit co-labeling with K15 (green) despite often being in very close proximity. Images captured using a Zeiss Laser Scanning Confocal Microscope Meta 510 system.

Figure AS3. Examination of skeletal myocytes following VE-cadherin cell lineage tracinghigh resolution confocal image. Hamstring tissue was harvested from VEcad-CreER ${ }^{T 2} /$ Rosa-flox-STOP-tdTomato transgenic mice 3 months post-tamoxifen treatment initiation. Fixed samples were sectioned $(15 \mu \mathrm{m})$ onto slides, labeledwith Hoechst dye, mounted with coverslips, and imaged using an Olympus FV3000 Laser Scanning Confocal Microscope system. The displayed image was obtained from a series of 10 $\mu \mathrm{m} z$-stacked planes compressed into a maximum intensity projection image showing tdTomato (red) and Hoechst (blue) channels.

Figure AS4. VE-cadherin-traced cerebellar granule neurons confocal microscopy high resolution tiled image. Tissue was harvested from $\mathrm{VEcad}-\mathrm{CreER} \mathrm{R}^{T 2} / \mathrm{Rosa}$ flox-STOP-tdTomato transgenic mice 3 months post-tamoxifen treatment initiation. The cryosectioned tissue $(15 \mu \mathrm{m})$ was fixed, labeled with Hoechst dye, and mounted with a coverslip. A tiled image was captured for $10 \mu \mathrm{m}$ z-stacked planes and stitchedusing a Zeiss 880 Airy Scan system. Hoechst dye (blue) and tdTomato (red) channels are displayed in the maximum intensity projection image shown.

Figure AS5 Confocal microscopy orthogonal analysis of VE-cadherin-traced pancreaticislet cells presented in Figure A5. (A) Immunohistochemistry for insulin/tdTomato-co-labeled cell (arrowhead) assessed by orthogonal reconstruction from confocal stacked images. (B) Almost all tdTomato positive islet cells examined are insulin (green)-negative despite very close physical proximity. (C) No glucagon (green)/tdTomato co-labeled cells were observed in any pancreatic section examined. Arrowheads indicate a point of assessment. A Zeiss Laser Scanning Confocal Microscope Meta 510 system was used to capture andanalyze the images shown. 103

Figure AS6 VE-cadherin cell lineage tracing high-resolution confocal image of pancreatic acini.Pancreatic tissue was harvested from VEcadCreER ${ }^{T 2} /$ Rosa-flox-STOP-tdTomato transgenic mice 3 months posttamoxifen treatment initiation. Tissue samples were fixed, cryosectioned $(15 \mu \mathrm{m})$ onto slides, stained with Hoechst dye and then mounted with coverslips. The displayed image was captured by an Olympus FV3000 Laser Scanning Confocal Microscope system from $10 \mu \mathrm{m}$ z-stacked planes andrepresented as a maximum intensity projection. The merged image shows tdTomato (red) and Hoechst (blue) channels. 
Figure B1. Inhibitory pathway of colchicine.Colchicine inhibits activation of purinergic $\mathrm{P} 2 \mathrm{X} 2 / \mathrm{P} 2 \mathrm{X} 7$ receptors and blocks cation uptake and subsequent pro-inflammatory signaling cascades without affecting cell death. Colchicine also inhibits the NALP3 inflammasome pathway, the Rho/ROCK pathway via cytoskeleton rearrangement, and inhibits release of ROS, NO and TNFa. Figure 1 was created using BioRender.com (adapted from [36]

Figure B2. Structures of folic acid and its analogs methotrexate and aminopterin..124

Figure B3. Metabolic pathways of methotrexate. a) Methotrexate inhibits dihydrofolate reductase (DHFR) and prevents conversion of dihydrobiopterin $\left(\mathrm{BH}_{2}\right)$ to tetrahydrobiopterin $\left(\mathrm{BH}_{4}\right)$, leading to uncoupling of NO synthase. b) Methotrexate inhibits AICAR transformylase, leading to increased adenosine levels and subsequent antiinflammatory responses. c) Methotrexate stimulates lincRNA-p21 expression, leading to increased apoptotic gene expression and subsequent anti-inflammatory responses.

Figure B4. Autophagy mechanism and associated gene dysfunctions. Autophagy is a cellular mechanism by which metabolites, organelles, proteins and protein aggregates are enveloped by a vesicular membrane to form an autophagosome. This autophagosome is trafficked to a lysosome where fusion occurs, and lysosomal degradative enzymes break down the cargo. Dysfunction in several genes associated with neurodegenerative diseases have been implicated and are known to disrupt autophagy. Figure 4 image created using BioRender.com. .133

Figure B5. NRF2 signaling and inflammation. Under basal conditions, NRF2 is bound to its repressor, KEAP1 and ultimately degraded by the proteasome following ubiquination. However, under oxidative stress, free NRF2 translocates to the nucleus and dimerizes with small MAF family proteins. This complex binds to and promotes the expression of genes with an antioxidant response element, such as HO-1. HO-1 directly inhibits proinflammatory cytokines while upregulating anti-inflammatory cytokines as well as catalyzing the breakdown of heme into carbon monoxide, free iron, and biliverdin. Carbon monoxide is an inhibitor of the NFKB pathway, resulting in an overall decrease of pro-inflammatory cytokines. The figure B5 image was created using BioRender.com. .139

Figure B6. The structure for dimethyl fumarate 140 


\section{LIST OF ABBREVIATIONS}

\begin{tabular}{|c|c|}
\hline mTOR & Mammalian Target of Rapamycin \\
\hline PPAR & Peroxisome Proliferator-Activated Receptor \\
\hline ULK & Unc-51-Like Kinase \\
\hline Atg13 & Autophagy Related Protein 13 \\
\hline $\operatorname{Atg} 101$ & Autophagy Related Protein 101 \\
\hline FIP200 & RB1-Inducable Coiled-Coil Protein 1 \\
\hline VPS34 & Vacuolar Protein Sorting Protein 34 \\
\hline PE & Phosphatidylethanolamine \\
\hline LC3 & Microtubule Associated Light Protein Light Chain 3 \\
\hline $\operatorname{Atg} 7$ & Autophagy Related protein 7 \\
\hline Atg3 & Autophagy Related Protein 3 \\
\hline $\operatorname{Atg} 12$ & Autophagy Related Protein 12 \\
\hline $\operatorname{Atg} 10$ & Autophagy Related Protein 10 \\
\hline $\operatorname{Atg} 16 \mathrm{~L}$ & Autophagy Related 16 Like Protein 1 \\
\hline Sqstm1 & Sequestome 1 \\
\hline MTMR3 & Myotubularin 3 \\
\hline UVRAG & UV Resistance Associated Gene \\
\hline Rab7 & Ras Related Protein 7 \\
\hline SNAREs & Snap Receptor Proteins \\
\hline LAMPs & Lysosome Associated Membrane Glycoproteins \\
\hline
\end{tabular}




\begin{tabular}{|c|c|}
\hline Avt3 & Vacuolar Amnio Acid Transporter 3 \\
\hline Avt4 & Vacuolar Amino Acid Transporter 4 \\
\hline PI3K & Phosphatidylinositol 3 Kinase \\
\hline PIP3 & Phosphatidylinositol-3,4,5-Triphosphate \\
\hline AKT & Protein Kinase B \\
\hline PTEN & Phosphatase And Tensin Homolog \\
\hline SOD1 & Superoxide Dismutase \\
\hline Atg9 & Autophagy Related Protein 9 \\
\hline Pink1 & PTEN Induced Kinase 1 \\
\hline ATP13A2 & Lysosomal Type 5 P-Type Atpase \\
\hline PIP2 & Phosphatidylinositol-4,5-Bisphosphate \\
\hline PDK1 & Phosphoinositide Dependent Kinase 1 \\
\hline $\mathrm{TSC}$ & Tuberous Sclerosis \\
\hline mTORC1 & Mtor Complex 1 \\
\hline PAS & Pre-Autophagosomal Structure \\
\hline Alfy & Autophagy Linked FYVE Protein \\
\hline BCL-2 & B-Cell Lymphoma Protein 2 \\
\hline JNK & C-Jun Terminal Kinase \\
\hline $\mathrm{NADPH}$ & Nicotinamide Adenine Dinucleotide Phosphate \\
\hline RXR & Retinoid Receptor X \\
\hline LBD & Ligand Binding Domain \\
\hline TFEB & Transcription Factor EB \\
\hline ATG & Adipose Triglyceride Lipase \\
\hline
\end{tabular}




\begin{tabular}{|c|c|}
\hline FOXO1 & Forkhead Homeobox Protein O1 \\
\hline LIPA & Lysosomal Acid Lipase \\
\hline AMPK & Adenosine Monophosphate Kinase \\
\hline $\mathrm{PA}$ & Palmitic Acid \\
\hline $\mathrm{PKC}$ & Protein Kinase C \\
\hline MAPK & Mitogen Activated Protein Kinase \\
\hline ERK & Extracellular Signal-Regulated Kinase \\
\hline MA & Myristic Acid \\
\hline DHA & Docosahexaenoic Acid \\
\hline $\mathrm{PD}$ & Parkinson's Disease. \\
\hline FABP & Fatty Acid Binding Protein. \\
\hline ATG5 & Autophagy Regulating Protein 5. \\
\hline RA & All Trans-Retinoic Acid \\
\hline AA & Arachidonic Acid. \\
\hline $5 \mathrm{OE}$ & 5-Oxo-eicosatetraenoic acid. \\
\hline HSA & Hydroxystearic Acid. \\
\hline SA & Stearic Acid. \\
\hline PA & Palmitic Acid \\
\hline OXER1 & Oxoeicosanoid Receptor 1. \\
\hline TAM & Tamoxifen. \\
\hline CNS & Central Nervous System. \\
\hline GCL & Granule Cell Layer \\
\hline GL & Glomerular Layer. \\
\hline
\end{tabular}




\begin{tabular}{|c|c|}
\hline LV & Lateral Ventricle. \\
\hline $4 \mathrm{~V}$ & Fourth Ventricle. \\
\hline CGN & Cerebellar Granule Neuron \\
\hline SVZ & Subventricular Zone. \\
\hline 4-OHT & 4-Hydroxy-Tamoxifen \\
\hline AAV9 & Adeno-Associated Virus. \\
\hline FDA & Food and Drug Administration. \\
\hline GWAS & Genome-Wide Association Studies. \\
\hline SPIDR & Small Molecule Peptide Influenced Drug Repurposing. \\
\hline UMLS & Unified Medical Language System. \\
\hline HTS & High Throughput Screening \\
\hline SNP & Single Nucleotide Polymorphism. \\
\hline ADAMTS7 & Disintegrin and mettaloproteinase with Thrombospondin Motifs \\
\hline TS7. & \\
\hline CAD & Coronary Artery Disease. \\
\hline GAMPMS & Genetic Algorithm Managed Peptide Mutant Screening \\
\hline $\mathrm{nAChR}$ & Nicotinic Acetylcholine Receptor \\
\hline SPR & Surface Plasmon Resonance \\
\hline NMR & Nuclear Magnetic Resonance. \\
\hline NO & Nitric Oxide. \\
\hline ROS & Reactive Oxygen Species. \\
\hline AICAR & Aminoimidazole-4-carboxamide ribonucleoride. \\
\hline $\mathrm{NF \kappa B}$ & Nuclear Factor kappa B. \\
\hline
\end{tabular}




\begin{tabular}{|c|c|}
\hline TNF- $\alpha$ & Tumor Necrosis Factor alpha. \\
\hline IL-6 & Interleukin 6. \\
\hline TCZ & Tocilizumab. \\
\hline NET & Network of chromatin and granule. \\
\hline PAH & Pulmonary Arterial Hypertension. \\
\hline IL-1 & Interleukin 1. \\
\hline IL-1R & Interleukin 1 Receptor. \\
\hline MyD88 & Myeloid Differentiation Primary Response Protein 88. \\
\hline LTA4H & Leukotriene A4 Hydrolase. \\
\hline cAMP & Cyclic Adenosine Monophosphate. \\
\hline PIP3 & Phosphatidylinositol-3-posphate. \\
\hline FRAP1 & FK506-Binding Protein 12-Rapamycin-Associated Protein 1. \\
\hline NRF2 & Nuclear factor (erythroid derived 2)- like 2. \\
\hline HO-1 & Heme Oxygenase 1. \\
\hline GST & Glutathione Synthase. \\
\hline UDP & UDP-Glucoronosyltransferase. \\
\hline $\mathrm{CO}$ & Carbon Monoxide. \\
\hline DMF & Dimethyl Fumurate. \\
\hline MS & Multiple Sclerosis. \\
\hline IL-4 & Interleukin 4. \\
\hline iNOS & Inducible Nitric Oxide Synthase. \\
\hline
\end{tabular}




\title{
CHAPTER ONE: A REVIEW OF SIGNALING AND OTHER ROLES OF LIPIDS IN
}

\begin{abstract}
AUTOPHAGY
The process of autophagy is integral to cellular function. In this process, proteins, organelles, and metabolites are engulfed in a lipid vesicle and trafficked to a lysosome for degradation. Its central role in protein and organelle homeostasis has piqued interest for autophagy dysfunction as a driver of pathology for a number of diseases including cancer, muscular disorders, neurological disorders, and non-alcoholic fatty liver disease. For much of its history, the study of autophagy has centered around proteins, however, due to advances in mass spectrometry and refined methodologies, the role of lipids in this essential cellular process has become more apparent. This review discusses the diverse endogenous lipid compounds shown to mediate autophagy. Downstream lipid signaling pathways are also reviewed in the context of autophagy regulation. Specific focus is placed upon the Mammalian Target of Rapamycin (mTOR) and Peroxisome Proliferator Activated Receptor (PPAR) signaling pathways as integration hubs for lipid regulation of autophagy.
\end{abstract}

\section{Introduction}

Autophagy is a process by which proteins, organelles, and metabolites are broken down and turned over often as a response to starvation or as a means to protect the cell from damage. Autophagy pathways come in three forms, macroautophagy, microautophagy, and chaperone-mediated autophagy ${ }^{1}$. Of these, macroautophagy is the 
best characterized and most well understood. Macroautophagy (hereafter referred to as autophagy) was originally studied in yeast and involves the formation of lipid vesicles known as autophagosomes that engulf cargo to be degraded. Once formed, the autophagosome is trafficked to a lysosome and a fusion event occurs resulting in the degradation of the cargo within the autophagosome (Figure 1.1) ${ }^{1}$.

Autophagy is delineated into key events: initiation, nucleation, elongation, and formation of a mature autophagosome, fusion of the autophagosome with a

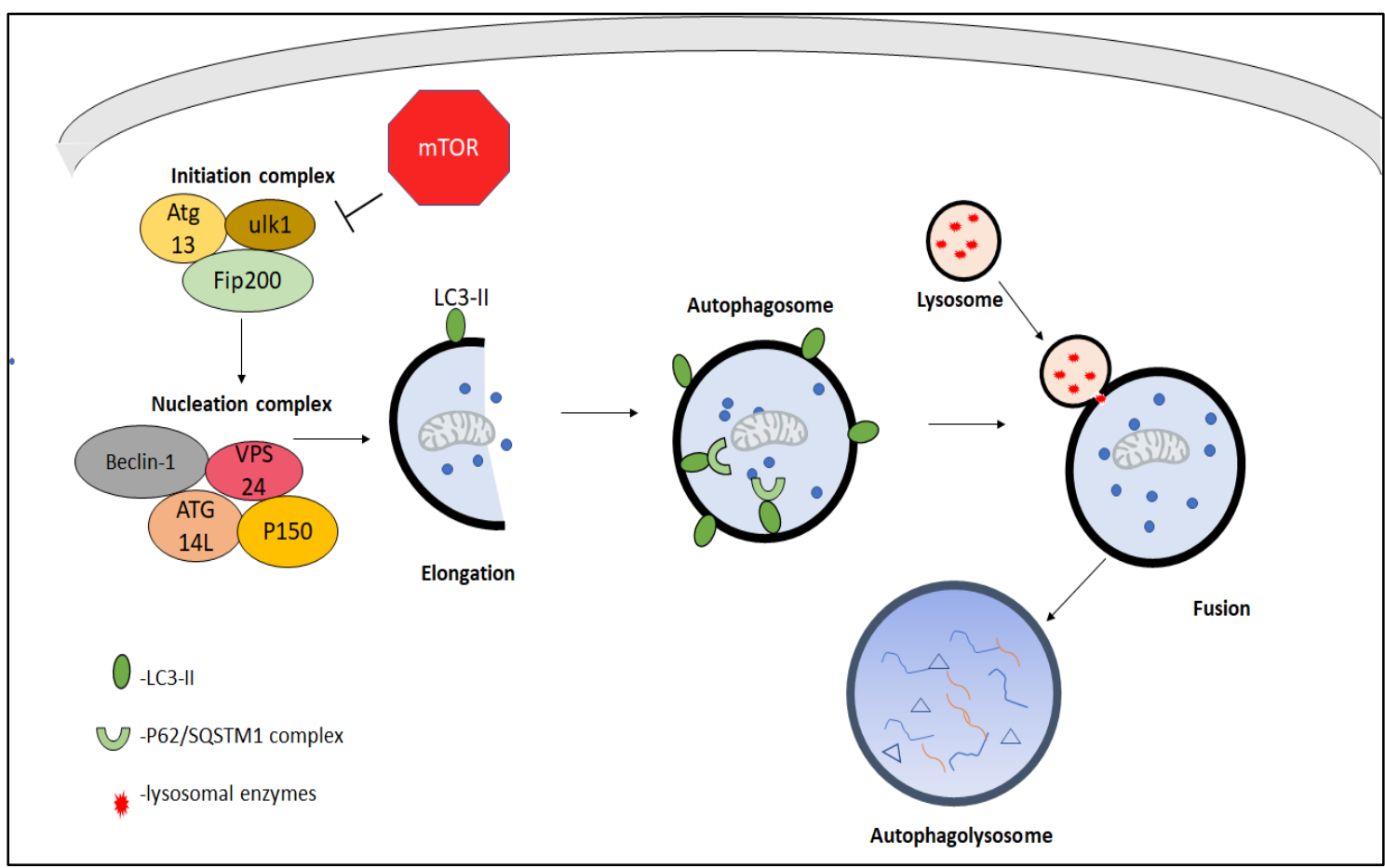

Figure 1.1 Autophagy mechanism. Autophagy is a cellular mechanism by which metabolites, organelles, proteins, and protein aggregates are enveloped by a vesicular membrane to form an autophagosome. The autophagosome is trafficked to a lysosome where fusion occurs, and lysosomal degradative enzymes break down the cargo. 
lysosome, and degradation of cargo. Initiation of autophagy is tightly regulated by the mTOR complex $1^{2}$. When the cell is in a nutrient-rich state, mTORC1 is active and autophagy is suppressed, however during nutrient-poor conditions, mTOR is inhibited which allows for the formation of Unc-51 like kinase (ULK) initiation complex composed of ULK kinases, autophagy related protein 13 (Atg13), Autophagy related protein 101 (Atg101), and RB1-inducable coiled-coil protein 1 (FIP200) ${ }^{3}$. Furthermore, ULK-1 also activates a second complex composed of Beclin1-vacuolar protein sorting protein 34 (VPS34)-autophagy related protein 14 (Atg14L)-P150, which produces phosphatidylinositol-3-phosphate (PI3P) (Figure 1.1) ${ }^{4}$. This complex is responsible for autophagic vesicles budding from the endoplasmic reticulum and forming a structure known as an omegasome. In mammals this is the site responsible for the nucleation of autophagosomes ${ }^{5}$. Next, phosphatidylethanolamine (PE) is conjugated to microtubule associated light protein light chain 3 (LC3) by autophagy related protein 7 (Atg7) and autophagy related protein 3 (Atg3), which are ubiquitin-like conjugating enzymes. Then the conjugated PE-LC3 is inserted into the autophagosome membrane ${ }^{6}$. In addition, autophagy related protein $12(\mathrm{Atg} 12)$ is conjugated to autophagy related protein 5 (Atg5) by Atg7 and autophagy related protein 10 (Atg10) also in a ubiquitin like manner ${ }^{7}$. Atg12-Atg5 interacts with autophagy related 16 like protein (Atg16L) and promotes elongation ${ }^{8}$. Meanwhile cargo is selected by ubiquitination and interaction with cargo receptor proteins p62/ sequestome 1 (sqstm1). Cargo bound to p62 then binds to the p62 interacting regions of $\mathrm{LC} 3{ }^{9}$. After the cargo is selected, the autophagosome matures by disassembling the autophagy related proteins from the outer layer with the help of myotubularin 3 (MTMR3), a PI3P phosphatase ${ }^{10}$. Once matured, the autophagosome will 
fuse with early and late endosomes as well as with lysosomes this is mediated by Rubicon, UV resistance-associated gene (UVRAG), Ras related protein 7 (Rab7), snap receptor proteins (SNAREs), and Lysosome-associated membrane glycoproteins (LAMPs) ${ }^{11-13}$. Once fusion with a lysosome is complete, the cargo is degraded. In mammals, lysosomal hydrolases break down cargo. Beneficial components, such as amino acids are then returned to the cytosol via amino acid effluxers such as vacuolar amino acid transporter 3 (Avt3) and vacuolar amino acid transporter 4 (Avt4) ${ }^{14}$.

\section{Lipid Signaling Directs Autophagy}

While autophagy has been studied extensively through the years, the role of lipids in this process is underrepresented. Historically, working with lipids has presented a challenge, leading to emphasis on work that primarily focused upon protein contributions. However, recent advances in both mass spectrometry capabilities and methodologies have spurred considerable progress in the study of lipids. For example, lipophagy, the targeted breakdown of lipid droplets by autophagic pathways, is currently being studied in the context of non-alcoholic fatty liver disease, aging, and cancer. It is becoming more apparent that lipids play a prominent role in autophagy. mTOR, the master regulator of cell growth, metabolism, and autophagy is itself a part of a signaling cascade in which lipid phosphoinositides are involved. In addition, Peroxisome Proliferator-activating factors (PPARs), are nuclear receptors which respond to lipid signals and have been implicated in the control of autophagy and autophagy related genes. For all of these reasons, this review seeks to provide a comprehensive overview of the growing field of lipid signaling. In the subsequent sections of this article, we discuss 
the different lipid signaling pathways known to regulate autophagy and their implications in disease states.

\section{Impaired Autophagy in Human Disease}

Autophagy is of considerable interest as a potential target for treatment in many diseases that include cancer, muscular disorders, and neurodegenerative disease. The fundamental role for organelle, particularly mitochondria, and biomolecule turnover by autophagy provides a broad influence of this process in cellular physiology. In addition, autophagy is the only known cellular process for removing protein aggregates making study of this process of considerable interest in protein aggregation disorders which coincide with numerous neurodegenerative diseases. Therefore, understanding and developing tools to manipulate autophagy could yield widespread therapeutic benefits.

Due to its regulation by mammalian target of rapamycin (mTOR), autophagy is intimately involved in growth, cell death and cytoprotective processes. As a result, there is great interest in harnessing this process in the context of cancer. In early stages, suppression of autophagy is believed to facilitate the uncontrolled growth ${ }^{15}$. In later stages, cells may require increased autophagy in low-oxygen and low-nutrient conditions, such as those seen in tumors ${ }^{16}$. Autophagy also can protect tumors from ionizing radiation by helping to remove damaged organelles and proteins ${ }^{17}$. Dysfunction in the phosphatidylinositol-3-kinase (PI3K)-protein kinase B (Akt)-mTOR pathway has been commonly seen to result in altered autophagy. This pathway, when active, suppresses autophagy and uses lipid signaling molecules such as phosphatidylinositol-3,4,5triphosphate $\left(\mathrm{PIP}_{3}\right)$ as key signal transducers ${ }^{18}$. Mutations in phosphatase and tensin homolog (PTEN), a phosphatase that antagonizes PI3K and causes positive regulation of 
autophagy, result in aberrant inhibition of autophagy that has been associated with excessive growth and tumor formation ${ }^{19}$. Another common mutation in cancers that leads to autophagy dysfunction is Beclin-1. A high percentage of human breast, ovarian, and prostate cancers have a heterozygous mutation in this gene. Beclin-1 is a part of the initiation complex responsible for activating lipid kinases required for the formation of autophagosomes. In breast carcinoma cell line MCF7, it has been established that Beclin1 expression is below detectable limits, and transfection of the Beclin-1 gene upregulates autophagy ${ }^{15}$. Studies have also shown that mice with a heterozygous deletion of Beclin-1 are more susceptible to developing tumors ${ }^{20,21}$. This is further evidence of the role of beclin- 1 and autophagy play in cancer.

Autophagy has also been implicated in muscular disorders. It is common for autophagy to play an important role in post-mitotic cells, such as muscle cells and neurons due to the potential for damage from accumulation of dysfunctional or toxic molecules, protein or organelles. Vacuolar myopathy is a type of muscular disease in which the structure of lysosomes is abnormal either from a deficiency in lysosomal 


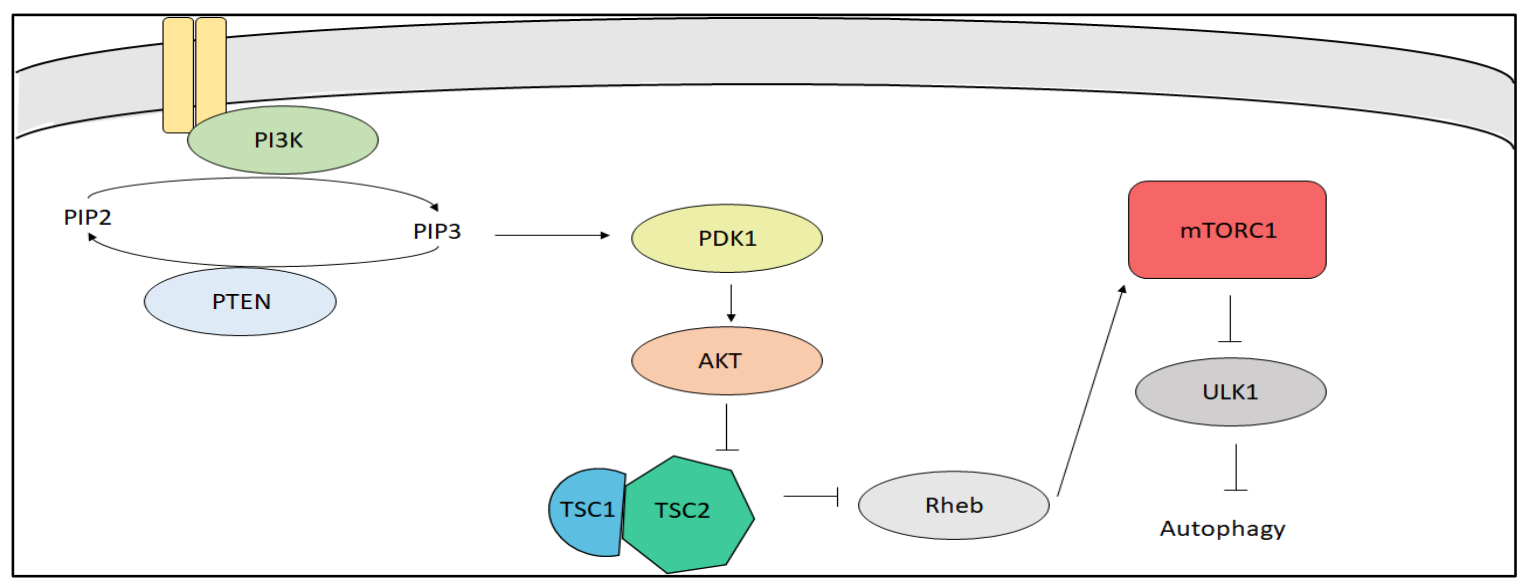

Figure 1.2 PI3K-mTOR autophagy pathway utilizes PIP3 lipid signaling. PI3K converts the lipid PIP2 to PIP3. PIP3 mediates the phosphorylation of PDK1 causing the activation of AKT. AKT inhibits the activation of the TSC1/2 complex by phosphorylating TSC2. The inhibition of the TSC1/2 complex results in inhibition of Rheb GTPase which in turn activates mTORC1. MTORC1 inhibits the activation of the ULK activation complex leading to an inhibition of autophagy. Inversely, phosphatase activity of PTEN converts PIP3 to PIP2 which suppresses the activation of PDK1 and downstream AKT. Inhibited AKT cannot suppress the TSC1/2 complex allowing Rheb GTPase to remain active. An active Rheb results in inhibition of $m$ TORC1 and an activation of the ULK1 complex

enzymes or a deficiency in lysosomal membrane proteins ${ }^{22}$. Therefore, it is not surprising that diseases in which lysosomal function is affected also result in the altered autophagy. In fact, an accumulation of autophagosomes is typically required to diagnose vacuolar myopathies ${ }^{23}$. In addition, an autophagy related gene has been associated with a vacuolar myopathy known as Danon's disease. In Danon's disease, mutations in lysosome associated membrane protein 2 (LAMP-2) have been identified ${ }^{23}$. LAMP-2 is a lysosomal membrane protein whose function is still not fully understood. However, studies in which the Lamp2 gene is deleted in mice result in a Danon's disease-like phenotype and an accumulation of autophagosomes ${ }^{23}$.

Autophagy has long been thought to play an important role in neurodegenerative disorders. A prominent hallmark of these diseases is the accumulation of protein 
aggregates associated with neuronal loss in the brain. Noted examples include: aSynuclein Lewy bodies in Parkinson's disease, Tau neurofibrillary tangles in Alzheimer's disease, Superoxide dismutase 1 (SOD1)-mediated aggregates in Amyotrophic Lateral Sclerosis, and mutant Huntingtin protein aggregates in Huntington's disease ${ }^{24-28}$. It is speculated that these aggregates may be substrates for autophagy, and that in these disease states, autophagy is disrupted. Several proteins have been identified and are linked to dysfunction in various steps of autophagy in each of these diseases. For example, in Alzheimer's disease, autophagy induction is disrupted by reduced expression of Beclin- ${ }^{29}$. In Parkinson's disease, an overexpression of a-Synuclein causes the inhibition of GTPase Rab1. This inhibition is responsible for the mislocalization of autophagy related protein $9(\operatorname{Atg} 9)$, a protein involved in the formation of autophagosomes ${ }^{30}$. Also, PTEN induced kinase 1(PINK1) and Parkin are proteins involved in the recognition of damaged mitochondria normally targeted for degradation mitophagy. Loss of function mutations in these proteins can prevent the necessary destruction of damaged mitochondria through autophagy resulting in cell death ${ }^{31,32}$. In addition, the park 9 gene encodes lysosomal type 5 p-type ATPase (ATP13A2). Autosomal recessive mutations in the park 9 gene result in levodopa-responsive earlyonset Parkinson's Disease. This loss of function mutation is responsible for aberrant expression of zinc transporters and an impairment in the ability for $\mathrm{Zn} 2+$ to enter lysosomal vesicles resulting in an induction of reactive oxygen species and impairment of mitochondrial function ${ }^{33}$. In Huntington's disease, mutant huntingtin protein is known to affect several stages in autophagy ${ }^{32}$. Many research groups have reported changes in 
expression of mRNA corresponding to genes in the autophagic pathway ${ }^{34}$. In addition, a polymorphism in the ATG7 gene has been linked to early onset Huntington's isease ${ }^{35}$.

\section{Phospholipids, Sphingolipids, and mTOR Signaling}

Phosphoinositides are a class of phospholipids derived from phosphatidylinositol, which is found in the inner layer of the cell membrane and are commonly used by the cell as signaling molecules ${ }^{36}$. They play a major role in the regulation of autophagy through phosphorylation and dephosphorylation at the 3,4 and 5-hydroxyl positions of the inositol ring. They control the pathway that directly regulates $\mathrm{mTOR}{ }^{37}$. mTOR itself is a master regulator of growth, anabolic processes, and autophagy. Generally, mTOR is activated in response to insulin, other nutrients such as amino acids or triglycerides, and growth factors. When active, mTOR promotes growth and suppresses autophagy. In response to starvation, the cell inhibits mTOR and autophagy is promoted ${ }^{2}$.

The canonical signaling pathway that controls autophagy functions through $\mathrm{PIP}_{3}$. The pathway begins as a response to insulin, other nutrients, or growth factors ${ }^{38}$. Phosphoinositide 3-kinases convert phosphatidylinositol 4,5- bisphosphate ( $\left.\mathrm{PIP}_{2}\right)$ to $\mathrm{PIP}_{3}$. $\mathrm{PIP}_{3}$ activates phosphoinositide dependent kinase-1 (PDK1) which in turn phosphorylates Akt $^{39,40}$. Akt then phosphorylates tuberous sclerosis 2 (TSC2) which results in the inhibition of the tuberous sclerosis $1 / 2(\mathrm{TSC} 1 / 2)$ complex ${ }^{41}$. When inhibited, TSC1/2 cannot activate Rheb GTPase activity permitting activation of mTOR. When bound to GTP, Rheb mediates the activation of mTOR complex 1 (mTORC1) which, in turn, inhibits autophagy ${ }^{42}$. Activated mTORC1 inhibits autophagy by inhibiting the ULK1 initiation complex. Pro-autophagy signals result in ULK1 dissociation from mTOR and autophagy initiation is facilitated (Figure 1.2) ${ }^{43}$. 
PI3P also plays an integral role in the process of autophagy by interacting with VPS34 ${ }^{44}$. Originally identified and studied in yeast, VPS34 is a class III phosphatidylinositol 3 kinase. In yeast, VPS34 forms one of two complexes. VPS34 complex-I is composed of VPS34, VPS30/ATG6, VPS15, and ATG14 and is implicated in the initiation of the pre-autophagosomal structure (PAS) ${ }^{45,46}$. In Mammals, VPS34 is thought to play a similar role in autophagy initiation. However, it has been difficult to study in mouse models since pan knockouts of VPS34 are embryonically lethal, and there are no inhibitors specific to VPS34 necessitating the use of low specificity inhibitors, such as wortmannin or 3-MA ${ }^{47}$. Conditional knockout studies using cultured mice embryonic fibroblasts have shown that VPS34 is required for the formation of autophagosomes ${ }^{48}$. In addition, VPS34 is involved with mTOR regulation of autophagy. Studies using mice embryonic fibroblasts have shown that mTORC1 must be inactivated for the VPS34 initiation complex to be active and that mTORC1 is able to inhibit the phosphatidylinositol 3-kinase activity of this complex by phosphorylating ATG14 ${ }^{48}$. PI3P is also a component of the autophagosome. It has been observed to be enriched in the concave surface of early phagophores ${ }^{49}$. Because of this, PI3P is thought to facilitate the expansion and sealing of autophagosomes.

In addition to its function in the autophagosomal membrane, PI3P is thought to mediate selected cargo capture via its interaction with autophagy linked FYVE protein (Alfy), a nuclear scaffold protein with a FYVE domain that binds PI3P ${ }^{50}$. In the autophagic process, Alfy interacts with Ath5 and P62 through its WD40 domain and with PI3P through its FYVE domain. The high amount of PI3P in the inner membrane of autophagosomes is believed to act as a dock for this Alfy/Atg5/P62 complex in the 
selective engulfment of protein aggregates that results in aggregate clearance known as aggrephagy ${ }^{51}$.

Metabolites formed from the breakdown of phospholipids are also involved in autophagy. Phosphatidic acid is formed by the breakdown of phosphatidylcholine into choline and phosphatidic acid by Phospholipase $\mathrm{D}^{52}$. Phosphatidic acid plays a role in autophagy by inducing membrane curvature due to its cone shape. In addition, Phosphatidic acid is formed as a result of an absence of nutrients and serves as an inhibitor of mTORC1 thus acting as a positive regulator of autophagy ${ }^{53}$. Phosphatidic acid can also be converted into diacylglycerol by the Phosphatidic Acid Phosphatases which has other autophagy regulating properties ${ }^{54}$. Diacylglycerol modulates autophagy by activating Protein Kinase $\mathrm{C}$ which induces autophagy by disrupting the B-cell lymphoma protein 2 (Bcl-2)-Beclin-1 complex via c-Jun $\mathrm{N}$ terminal kinase (JNK) and Nicotinamide adenine dinucleotide phosphate (NADPH) oxidase ${ }^{54}$.

Sphingolipids are a class of lipids involved in several processes ranging from apoptosis to cell proliferation to differentiation, inflammation and autophagy ${ }^{55}$. In autophagy two sphingolipids have been found to play sizeable roles, ceramide and sphingosine -1-phosphate. Ceramide activates autophagy by inhibiting Akt and resulting in an inactivation of mTOR and an upregulation of Beclin-1 function ${ }^{56}$. Sphingosine-1phosphate is formed by the hydrolysis of ceramide into sphingosine followed by its phosphorylation by Sphingosine Kinases 1 and $2{ }^{57}$. Sphingosine Kinase 1 is activated by starvation signals and drives the formation of sphingosine-1-phosphate ${ }^{57}$. In addition, sphingosine kinase 1 inhibits mTORC1 independently of Akt while upregulating beclin-1 expression, ultimately promoting cell survival ${ }^{57}$. 


\section{Signaling Through the PPAR Family}

PPARs are a family of nuclear receptor proteins that act as transcription factors. There are 3 isoforms of PPARs in mammals, PPAR $\alpha, \operatorname{PPAR} \delta$, and PPAR $\gamma^{58}$. All 3 isoforms of PPARs must bind with a Retinoid Receptor X (RXR) and a lipid ligand in order to act as transcription factors (Figure 1.3) ${ }^{59}$. Generally, they have been reported to bind to oleic acid, linoleic acids, linolenic acids, prostaglandins, eicosanoids, and oxidized lipids with the help of fatty acid binding proteins which bind lipophilic ligands in the cytoplasm and shuttle them to their target PPAR ${ }^{60,61}$. PPARs bind to their ligands through the ligand binding domain (LBD). These domains consist of $12 \alpha$-helices arranged into an 'antiparallel helix sandwich' and a three-stranded antiparallel $\beta$-sheet. The ligand binding site is located in the core of the ligand binding domain that is formed by helices $3,5,7,11$, and 12 . The cavity formed by these helices is T-shaped ${ }^{62}$. In order for ligands to bind to PPAR- $\alpha$ or PPAR- $\gamma$ they must be able to form a U-shaped conformation, and in order to bind to PPAR-delta ligands must form an L-shaped conformation ${ }^{63}$. All PPARs isoforms have been shown to modulate autophagy in the context of different diseases and cellular responses.

PPAR $\alpha$ is primarily expressed in the liver, brown adipose tissue, heart, and kidney. It promotes uptake and catabolism of fatty acids by helping to express fatty acid transport and binding genes ${ }^{58} 64$. It has been thought to be involved in innate immune response during mycobacterium infection ${ }^{65}$. In studies with tuberculosis infected bone 


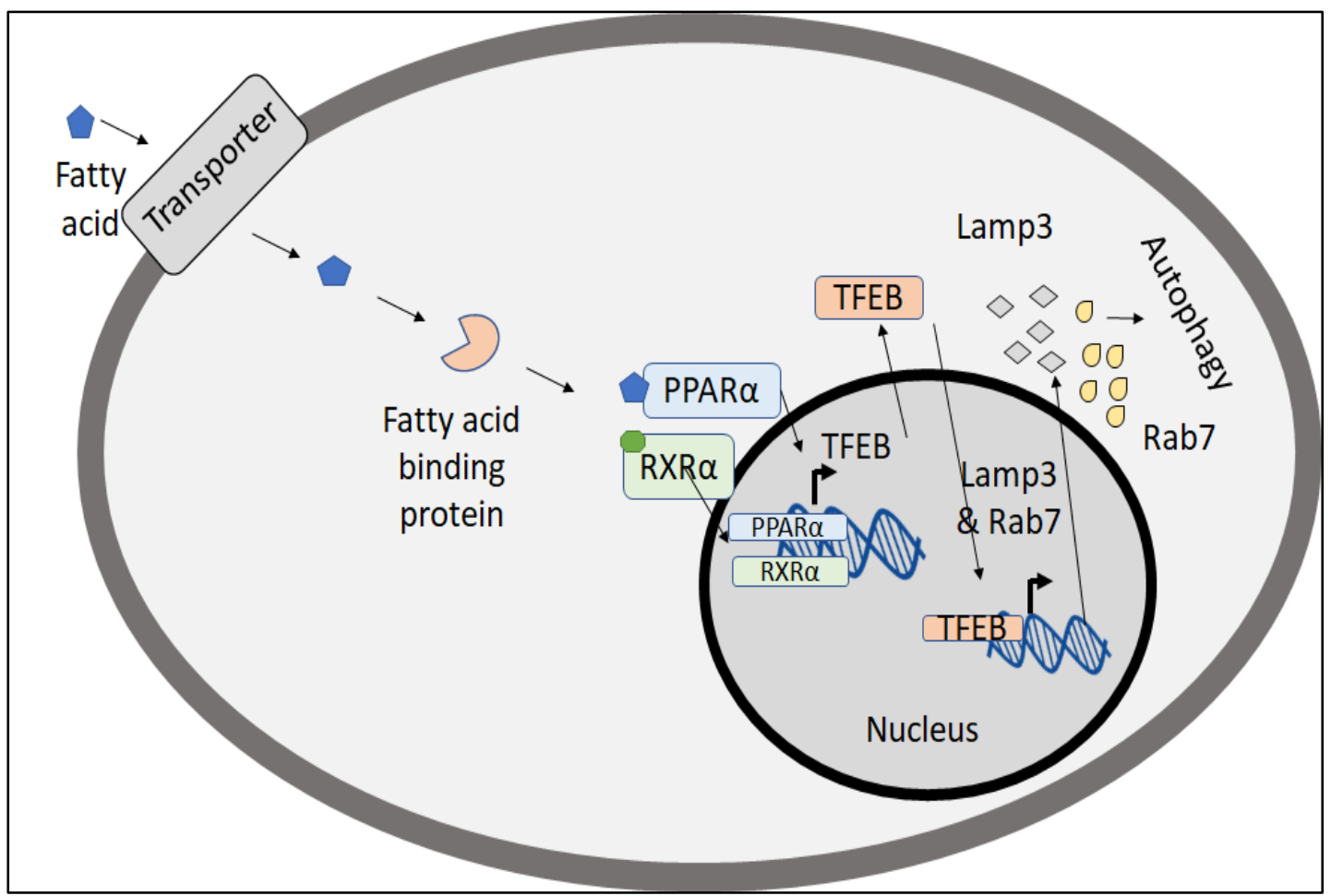

Figure 1.3 Overview of PPAR signaling and mechanism for PPARa-mediated autophagy activation in innate immune system. Initially, lipid molecule enters the cell and is quickly bound by a fatty acid binding protein. The fatty acid binding protein transports the lipid to a PPAR which in turn activates a corresponding RXR. The PPAR-RXR complex crosses into the nucleus and facilitate expression of required genes. In the case of tuberculosis infection, PPAR $\alpha$ upregulates the expression of TFEB which, in turn, drives the expression of autophagy related genes, LAMP3 and RAB7 thus stimulating autophagy.

derived macrophages, PPAR $\alpha$ were shown to stimulate autophagy and autophagosomal maturation, while suppressing inflammatory responses. It was determined that following PPAR $\alpha$ activation, Transcription Factor EB (TFEB) was activated and a series of autophagy and lysosomal genes were expressed such as LAMP3 and Rab7 ${ }^{65}$. Based on this work, it is thought that, in mycobacterial infections, such as tuberculosis, PPAR $\alpha$ is activated and in turn activates TFEB. Together they promote the expression of autophagy-related genes which stimulate autophagy (Figure 1.3). 
PPAR $\delta$ has high expression levels in the colon, small intestine, liver, heart lung and brain. It plays an important role in diseases such as diabetes, obesity, atherosclerosis, and cancer ${ }^{58,64}$. This is especially poignant, because there are great efforts in exploiting autophagy as possible treatments for cancer and diabetes. Studies in mice cells have shown a marked decrease in autophagic markers associated with the knockout of PPAR $\delta$ suggesting its involvement in autophagy ${ }^{66}$.

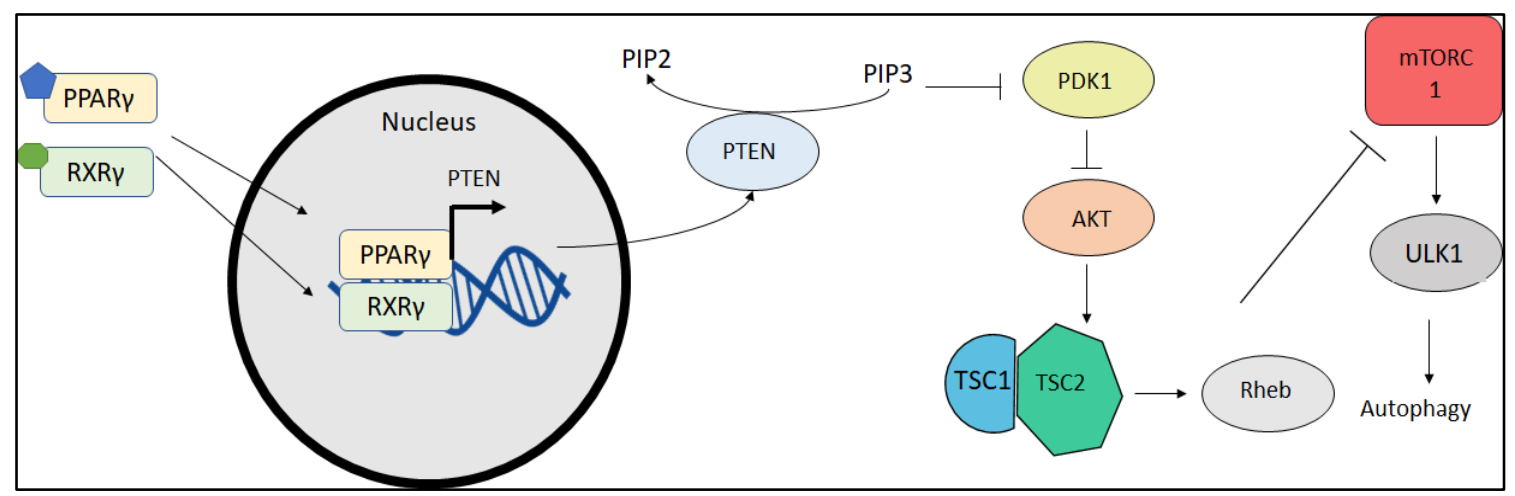

Figure 1.4 PPAR $\gamma$ mediated activation of autophagy. PPAR $\gamma$ promotes the expression of PTEN. High amounts of PTEN lead to lower concentrations of PI $(3,4,5) P 3$. Less PI $(3,4,5) P 3$ inhibits the activation of PDK1 and ultimately results in inhibition of mTORC1 which causes an activation of autophagy.

Finally, PPAR $\gamma$ is expressed in adipose tissue, the intestines and macrophages. It is usually involved in fatty acid storage, glucose uptake, and adipogenesis ${ }^{58,64}$. Because of its role controlling the availability of nutrients, there has been interest in targeting it as a treatment for cancer. In Colorectal cancer, studies with Caco-2, a common colorectal cancer cell line, have shown that activation of autophagy occurs following treatment with PPAR $\gamma$ agonist rosiglitazone ${ }^{67}$. In addition, inhibition of autophagy with 3-MA was observed to induce expression of PPAR $\gamma$. PPAR $\gamma$ was determined to cause the induction of PTEN, an antagonist to PI3K which dephosphorylates and reduces the concentration of $\mathrm{PIP}_{3}{ }^{67}$. This results in an overall inhibition of the mTOR pathway and induces 
autophagy. In the context of breast cancer, PPAR $\gamma$ has also been implicated to modulate autophagy. Activation of PPAR $\gamma$ by agonist troglitazone was shown to induce autophagy in MDA-MB231 cells as determined by the measurement of acidic vesicular organelles by staining with Acridine orange ${ }^{68}$. In addition, studies of constitutively active PPAR $\gamma$ suggest that it is sufficient for the activation of autophagy leading to the belief that autophagy acts to protect cancer cells (Figure 1.4) ${ }^{68}$.

\section{Lipid Metabolism and Autophagy}

Autophagy is intricately related to the metabolism of lipids, namely triglycerides, due to the fact that it responds to the presence or absence of nutrients in the cell. Furthermore, it is involved with the breakdown of stored lipids in the cell. Triglycerides are stored in organelles known as lipid droplets. They are used to generate energy, building blocks for membranes, and for lipid signaling ${ }^{69}$. Lipid droplets are broken down for use by the cell via lipophagy. This process is mediated by the GTPase Rab7 in hepatocytes and results in the release of free fatty acids under starvation conditions to be used as fuel in the mitochondria and undergo $\beta$-oxidation. Rab7 was shown to mediate the docking of autophagosome to lipid droplets facilitating their catabolism ${ }^{70}$. In addition, Adipose triglyceride lipase (ATGL) is a regulator of lipophagy. When knocked down in hepatocytes, ATGL causes decreased lipophagy. This ATGL signaling has been observed to occur through sirtuin 1. Together, these two proteins drive lipophagy and fatty acid oxidation ${ }^{71}$.

Additionally, breakdown of lipid droplets by lipophagy can be regulated by transcription factors. TFEB mediates the activation of PPAR alpha as a response to nutrient deprivation in order to activate lipophagy ${ }^{72}$ Additionally, forkhead homeobox 
protein $\mathrm{O} 1$ (FOXO1) becomes upregulated in nutrient restricted conditions and increases lipophagy of lipid droplets. The FOXO1 mediated lipophagy activation is facilitated by an increased expression of lysosomal acid lipase (LIPA) resulting in a release of free fatty acids through adenosine monophosphate kinase (AMPK)-dependent $\beta$-oxidation in adipocytes in nutrient restricted conditions ${ }^{73}$.

Conversely autophagy is linked to the biosynthesis of new triglycerides as well. Not only does autophagy drive the breakdown of lipid droplets, but it is also tied to the metabolic balance of liver triglycerides. Diets low in protein result in a reduced expression of autophagy receptor SQstm1 and increases the expression of LC3-II. This correlates to an induction of autophagy. It is speculated that, in the case of low protein availability, autophagy does not catabolize lipids and instead may help triglycerides to accumulate in the liver ${ }^{74}$. Additionally, Perilipin-2, a protein that associates with lipid droplets, has been observed to protect lipid droplets from autophagy. Perilipin-2 has been observed to inhibit lipogenesis and triglyceride production as well as upregulating autophagy when it is depleted in the cell ${ }^{75}$.

\section{Free Fatty Acids and Cholesterol}

Free fatty acids have also been implicated in the autophagic pathway. Although they usually act as nutrients, fatty acids can induce cell death when they accumulate in excessive levels in non-adipose cells and tissues. This is known as lipotoxicity and has been observed in diseases such as obesity, diabetes, and non-alcoholic fatty liver disease ${ }^{76}$. As a result, levels of free fatty acids are thought to be regulated inside the cell through lipophagy ${ }^{69}$. Palmitic acid (PA) and its effects on diabetes has been studied in rat pancreatic beta cell line INS-1 ${ }^{77}$. It was determined to trigger autophagy independently 
of the mTOR pathway. For instance, autophagy was shown to be promoted by stimulating JNK which leads to phosphorylation of Bcl-2 a resulting in its dissociation from Beclin-1 which in turn allowed for the initiation of autophagy and autophagosome formation ${ }^{77}$. In addition, protein kinase $\mathrm{C}$ (PKC) isoforms $\delta$, $\mathrm{a}$, and $\Theta$ have also been implicated in PA-mediated autophagy regulation ${ }^{78}$.

Studies suggest that in mice embryonic fibroblasts, PA, a saturated fatty acid can induce autophagy ${ }^{79}$. It was reported that palmitic acid was able to increase the amount of LC3, suggesting an induction of autophagy. However, there was no increase in phosphorylation of P70S6K or S6, two downstream proteins in the mTOR signaling pathway ${ }^{79}$. This suggests that PA induces autophagy independent of mTOR. PKCa was identified and shown to be involved in the autophagy inducing process. When it was knocked down with siRNA, LC3 detection fell ${ }^{79}$. Furthermore, studies show that while prolonged exposure to PA causes cell death, short term exposure induces autophagy, this suggests that autophagy is an important protective measure against lipotoxicity caused by $\mathrm{PA}^{79}$.

PA has been shown to modulate autophagy via a secondary signaling pathway. Its effects have been studied in the context of hepatic steatosis; a condition caused by high amounts of fat in the liver ${ }^{80}$. In hepatic steatosis, high lipid levels cause lipotoxicity. Non-alcoholic steatohepatitis mice were fed a high fat diet. These mice were shown to exhibit high autophagy mediated by $\mathrm{PA}^{80}$. In these studies, autophagy was determined to be regulated by the activation of mitogen activated protein kinase (MAPK), extracellular signal-regulated kinase (ERK), P38, JNK. Based on these studies, researchers concluded 
that JNK-1 has a lipo-apoptotic effect while JNK-2 promotes autophagy and has a cytoprotective effect (Figure 1.5) ${ }^{80}$.

In addition to PA, myristic acid (MA) has been studied for its autophagy regulating effects. Like palmitic acid, MA is a saturated fatty acid commonly used by the body as a nutrient. In the context of autophagy, MA has been found to promote overexpression of Beclin-1 gene BCN1 and increased conversion of LC3-I to LC3-II in mouse cardiomyocytes ${ }^{81}$. MA is thought to work to upregulate autophagy by producing C14-ceramide and upregulating ceramide synthase 5 (Figure 1.5) ${ }^{81}$.

Omega-3 polyunsaturated acids have been known to play a role in regulating autophagy. The most studied of these is docosahexaenoic acid (DHA). DHA is a component in many of the phospholipids that make up the cell membrane in the brain, skin, and retinal tissue ${ }^{82}$. It has been studied in myocardioblasts and various human cancer cell lines ${ }^{83-85}$. DHA has been implicated in the p53-AMPK-mTOR signaling 


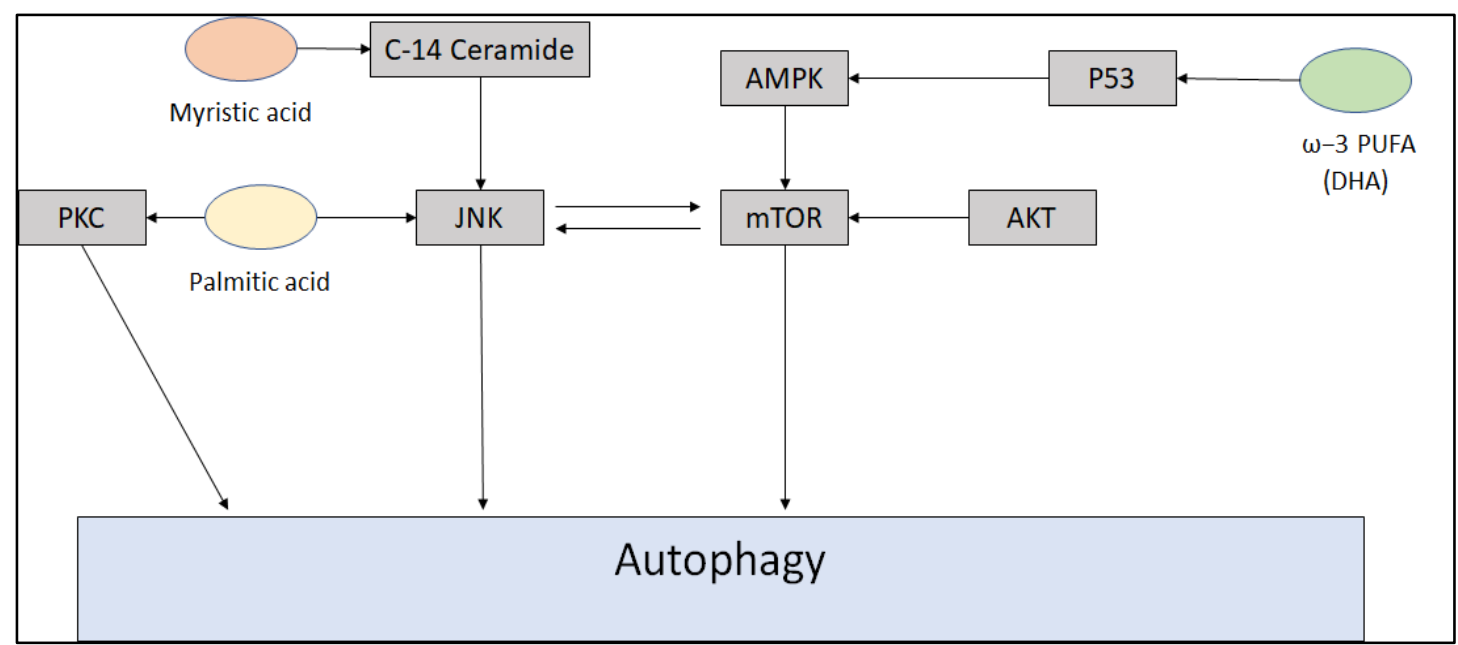

Figure 1.5 Overview of regulation of autophagy by free fatty acids. Palmitic acid stimulates autophagy by activating JNK and has been shown to interact with PKC isoforms which have been implicated in autophagy regulation. Myristic acid has been shown to upregulate autophagy by producing C-14 ceramide. DHA has been implicated in the P53-AMPK-mTOR pathway resulting in activation of autophagy.

pathway. It has been shown to work through AMPK to inhibit mTOR and induce autophagy in human cancer cells with wild type p53. ${ }^{86}$. However, In prostate cancer cells with mutant p53, DHA was observed to induce autophagy through the creation of mitochondrial reactive oxygen species that results in the inactivation of AKT and mTOR also resulting in an activation of autophagy (Figure 1.5) ${ }^{87}$.

Finally, autophagy is related to cholesterol biosynthesis inversely through the mTOR signaling pathway. When cholesterol biosynthesis is inhibited, there is an induction of autophagy. Inhibition of cholesterol biosynthesis by statin drugs such as, simvastatin has been linked to autophagy activation via the inhibition of the mTOR signaling pathway in human blood cancer cells. It was determined that the statin drug caused this activation only through cholesterol depletion when the cholesterol depleting agent, methyl- $\beta$-cyclodextrin also produced an activation of autophagy ${ }^{88}$. Likewise, a deficiency of transmembrane 7 superfamily member 2 (TM7SF2), a positive regulator of 
cholesterol biosynthesis, results in repression of autophagy. Removal of TMS7F2 causes increases in the expression of fatty acid degradative enzymes, decreased lipid accumulation and in turn decreased autophagy in mice exposed to Lipopolysaccharide ${ }^{89}$.

\section{Conclusion and Future Perspectives}

The aim of this review is to outline the current state in the field of lipid regulation of autophagy. To that end, this review has discussed a myriad of ways in which many lipids are involved in the process of autophagy and its regulation. All the lipids discussed along with their functions and effects on autophagy have been summarized in Table 1.1 (Table 1.1). Autophagy represents a cellular process that has implications for several important areas of study. Phospholipids and their derivatives have been shown to not only be an important part of the regulation of autophagy through the crucial mTORC1 signaling pathway, but also an integral part of the autophagy machinery. These lipids have also been shown to affect a variety of processes by acting through PPARs with possible implication in human disease. Finally, dietary lipids and cholesterol have been implicated in the regulation of autophagy both through the canonical mTOR pathway and alternative means.

The role lipid molecules play in autophagy represents the potential for many new avenues of research. Understanding it can give us a better, more holistic idea of this process which is central to many cellular functions and disease states. As our understanding of autophagy has grown, its activators and inhibitors have begun to appear as a novel area of drug development. As a result, it is necessary to fill in the gaps in our knowledge with regards lipid signaling. In the specific case of disease, it may be necessary to identify novel lipid molecules involved in autophagy. It may also be helpful 
to determine if there is a link between the signaling pathways described or if they all act independently. Finally, if autophagy is to be exploited as a potential treatment for disease, it would be necessary to evaluate the effects these lipid molecules and signaling pathways have on cellular functions related to autophagy.

Table 1.1 A Summary of Discussed Lipids Involved in Autophagy

\begin{tabular}{|c|c|c|}
\hline Lipid Name & & \\
\hline & is conjugated to LC3 and & required for autophagy \\
\hline Phosphatidylethanolamine (PE) & incorporates into autophagosome & progression \\
\hline $\begin{array}{l}\text { posphatidylinositol-3-phosphate } \\
\text { (PI3P) }\end{array}$ & $\begin{array}{l}\text { elongation of autophagosome } \\
\text { memebranes/ docking molecule } \\
\text { in selective cargo capture }\end{array}$ & $\begin{array}{l}\text { required for autophagy } \\
\text { progression }\end{array}$ \\
\hline $\begin{array}{l}\text { phosphatidylinositol-4,-5- } \\
\text { bisphosphate (PIP2) }\end{array}$ & $\begin{array}{c}\text { inhibits PI3K-AKT-mTOR } \\
\text { signaling pathway }\end{array}$ & activation of autophagy \\
\hline $\begin{array}{c}\text { phosphatidylinositol-3, 4, 5- } \\
\text { triphosphate (PIP3) }\end{array}$ & $\begin{array}{l}\text { activates PI3K-AKT-mTOR } \\
\text { signaling pathway }\end{array}$ & inhibition of autophagy \\
\hline phosphatidic acid & $\begin{array}{c}\text { induces membrane curvature in } \\
\text { autophagosomal vesicles/ } \\
\text { inhibits mTOR }\end{array}$ & $\begin{array}{c}\text { required for autophagy } \\
\text { progression/ activation } \\
\text { autophagy } \\
\end{array}$ \\
\hline diacylglycerol (DAG) & activates Protein Kinase $\mathrm{C}$ & activation of autophagy \\
\hline sphingosine-1-phosphate & $\begin{array}{c}\text { inhibits mTOR independently of } \\
\text { AKT }\end{array}$ & activation of autophagy \\
\hline ceramide & $\begin{array}{c}\text { inhibits AKT and, by extention, } \\
\text { mTOR }\end{array}$ & activation of autophagy \\
\hline triglycerides/lipid droplets & $\begin{array}{l}\text { abundance of triglycerides is } \\
\text { regulated by the cell through } \\
\text { autophagy of lipid droplets }\end{array}$ & $\begin{array}{l}\text { activation of autophagy/ } \\
\text { inhibition of autophagy }\end{array}$ \\
\hline palmitic acid (PA) & $\begin{array}{c}\text { activates Protein Kinase C/ } \\
\text { activates JNK }\end{array}$ & activation of autophagy \\
\hline myristic acid (MA) & $\begin{array}{l}\text { increases the production of } \\
\text { ceramide }\end{array}$ & activation of autophagy \\
\hline docosahexaenoic acid (DHA & $\begin{array}{l}\text { inhibits AMPK-mTOR pathway } \\
\text { through wild-type p53/ inhibits } \\
\text { AKT-mTOR through mutan p53 }\end{array}$ & activation of autophagy \\
\hline cholesterol & activates mTOR signaling & inhibition of autophagy \\
\hline
\end{tabular}

The technology we use to understand autophagy has increased greatly over the past years, and it has allowed our understanding to grow. However, there is still progress to be made especially with respect to the role lipids play in its regulation. For example, mass 
spectroscopy techniques have significantly improved in recent years allowing for the high throughput analysis and identification of lipids present in a system. Other advances that would facilitate new research could include an improved way of visualizing and even purifying autophagosomes and lysosomes. Current methods utilize immunoprecipitation or density gradient fractionation, both of which are known to result in significant levels of impurities, to isolate these subcellular structures. In conclusion, lipids play an important and diverse signaling role in autophagy regulation, and it is necessary to fully characterize lipid signal transduction pathways to better inform autophagy-based therapies. 


\section{CHAPTER TWO: LIPIDOMIC ANALYSIS OF FABP5-BOUND AUTOPHAGY REGULATORS IN DIFFERENTIATED SH-SY5Y CELLS}

The motor features of Parkinson's disease result from loss of dopaminergic neurons in the substantia nigra with autophagy dysfunction being closely linked to this disease. While a large body of work focusing on protein effectors of autophagy has been reported, regulation of autophagy by lipids has garnered far less attention. We, therefore, sought to identify endogenous lipid molecules that act as signaling mediators of autophagy. In order to accomplish this goal, we assessed the role of fatty acid binding protein (FABP) family members on autophagy. Knockdown of FABP5 resulted in suppression of autophagy in retinoic acid-differentiated SH-SY5Y cells suggesting an autophagic role for an interacting lipid. A lipidomic screen using FABP5, that is expressed by dopaminergic neurons, as "bait" was devised and implemented to identify lipid cargo. Our analysis has found significant hits that include 5-oxo-eicosatetraenoic acid (5OE) and its precursor metabolite, arachidonic acid (AA). Long chain fatty acids were also found to bind FABP5 that include stearic acid (SA), hydroxystearic acid (HSA) and palmitic acid (PA). Addition of 5OE, SA and HSA but not AA or PA, led to potent inhibition of autophagy in cultured dopaminergic cells. Correspondingly, heightened mTOR signaling was also observed following 5OE, HSA and SA treatment. To identify potential molecular mechanisms for autophagy inhibition by these lipids, RNA-Seq was performed which revealed both shared and divergent signaling pathways. These findings suggest a role for these lipids in modulating autophagy through diverse signaling 
pathways that may involve mTOR activity and could represent novel therapeutic targets for Parkinson's disease.

\section{Introduction}

\section{Autophagy and Parkinson's Disease}

Parkinson's disease (PD) is the second most frequent neurodegenerative disease that is characterized by motor system dysfunction caused by loss of dopaminergic neurons in the substantia nigra. Macroautophagy (subsequently denoted as autophagy) plays an important role in PD as evidenced by disease-causing mutations in genes directly involved in this essential cellular process ${ }^{90}$. Autophagy is a fundamental process for degrading macromolecules and organelles and is also the only cellular process known to remove large protein aggregates ${ }^{91}$. Therefore, defective autophagy is associated with the pathophysiology of PD due to the presence of hallmark Lewy body inclusions ${ }^{92}$. As a result, autophagy has been of a keen focus for the development of PD therapy.

Autophagy is a highly ordered catabolic process which begins by targeting material to be degraded through conjugation of p62 or by other mechanisms ${ }^{93}$. Once targeted, the material is engulfed by a lipid vesicle derived from the endoplasmic reticulum. The lipid vesicle is created through the propagation of phosphotidylethanolamine-Atg8 (LC3) lipoprotein complex. A vesicle with fully enveloped cargo will fuse with multivesicular bodies and then lysosomes possessing digestive enzymes to degrade the cargo into simple constituents that can be recycled for metabolic purposes. This process occurs constitutively within cells however the rate and targeted cargo can be modulated by numerous signaling pathways many of which converge on a master regulatory complex known as TORC1 ${ }^{94}$. 


\section{Regulation of Autophagy by Lipids}

Advances in our understanding of autophagy have occurred largely through the investigation of protein effectors of this process. However, little is known about the contribution of endogenous lipid compounds to autophagy regulation. Principal knowledge in this field has arisen from the study of AKT pathway activation by phosphatidylinositol phosphorylation, largely in the context of growth factor signaling and oncogenesis. Despite the reduced focus in this area, the involvement of phosphatidylinositol indicates that lipids are indeed critical signaling mediators for autophagy control. A limited understanding of other lipid contributors is likely due to the inherent physical properties of these biomolecules, which include enhanced oxidation, degradation and insolubility, rendering them difficult to study. Despite these limitations, lipid research is becoming more accessible, largely as a result of advancements in mass spectrometry resources (e.g., methods, databases) and the commercial availability of lipids. As such, we have employed these tools to identify autophagy-relevant lipids.

\section{FABP5 is an Intracellular Lipid Chaperone}

There are 10 members of the FABP family (FABP1-9 and 12) present in humans. As a group, these members transport fatty acids and other lipophilic compounds (e.g., eicosanoids and retinoids) within cells ${ }^{95,96}$. Individually, these proteins have both distinct and shared cargo lipids. The unique functionality of specific FABP members is also suggested by diverse tissue and developmental expression, as well as an association with human disease ${ }^{96,97}$. Therefore, this protein family represents a strong pool of potential bait proteins for the identification of autophagy-regulating lipids. A detailed examination of individual members has led to our specific interest in FABP5. Ewing et 
al., have provided evidence that FABP5 interacts with a known autophagy-regulating protein (ATG5) as one of 368 bait proteins in a mass spectrometry-based interactome screen ${ }^{98}$. In addition, FABP5 expression has been reported to modulate PI3K/AKT signaling ${ }^{99}$, which plays a central role in regulating autophagy via mTOR activity. Furthermore, FABP5 might have particular relevance to PD since it has been reported that its expression is highly enriched in nigral DA neurons ${ }^{100}$. As a result, we began our investigation into autophagy-regulating lipids by focusing on FABP5 interactors.

\section{Dopaminergic SH-SY5Y cells}

SH-SY5Y cells are human neuroblastoma cells derived from the SK-N-SH cell line ${ }^{101}$; they are a widely used cellular model for PD due to their physiologic similarity to dopaminergic neurons. These cells are commonly differentiated with all trans-retinoic acid (RA) into a DA neuron-like postmitotic cell capable of generating action potentials 102,103. Systems genomic analysis using transcriptome sequencing coupled with proteomic evaluation shows that SH-SY5Y cells have intact DA neuron signaling pathways ${ }^{104}$. RA-differentiated SH-SY5Y cells express mature DA neuron markers (TH, VMAT2, DAT, PITX3, RET, DRD2) and produce high levels of dopamine ${ }^{105}$. Evidence also indicates that these cells, like mature DA neurons, have intact DRD2 signaling ${ }^{105}$. Consequently, differentiated SH-SY5Y cells are commonly used to model DA neurons and were chosen for this study.

\section{Materials And Methods}

\section{$\underline{\text { Cell Culture }}$}

SH-SY5Y human neuroblastoma cells, from ATCC, were cultured in T175 tissue culture flasks and kept at $37^{\circ} \mathrm{C}$ with a $5 \% \mathrm{CO}_{2}$ environment. Cells were cultured in 
DMEM/F12 50/50 mix (Corning Cellgro) with L-glutamine, 15\% Fetal Bovine serum (Atlanta Biologicals), 1\% non-essential amino acids (Corning Cellgro), 1\% PenicillinStreptomycin solution (HycloneTM, 1000 units/mL Penicillin- 10000 $\mu \mathrm{g} / \mathrm{mL}$

Streptomycin) as previously described by Shipley, Mangold, \& Szpara ${ }^{106}$. Cells were passaged when the flasks reached $\sim 80 \%-90 \%$ confluency using $0.25 \%$ Trypsin, with 2.2mM EDTA and sodium bicarbonate (Corning). Cells at passage number 5-15 were used for experiments. For experiments, cells were plated on multi-well tissue culture plates at the necessary concentrations (e.g. 3 million cells per well on a 6-well plate). Before experimentation, the cells were treated with $10 \mu \mathrm{M}$ retinoic acid for 7 days, to differentiate them into dopaminergic neuron-like cells. HEK 293T Human Embryonic Kidney cells were grown in DMEM media with $1.0 \mathrm{~g} / \mathrm{L}$ glucose with L-glutamine and sodium pyruvate (VWR), 10\% Fetal Bovine Serum (Atlanta Biologicals), and 1\% Penicillin-Streptomycin solution (HycloneTM, 1000 units $/ \mathrm{mL}$ Penicillin- 10000 $\mu \mathrm{g} / \mathrm{mL}$ Streptomycin) and passaged as described for SH-SY5Y cells.

\section{$\underline{\text { Lipid Treatment of Cells }}$}

Stock solutions of 5-oxo-ETE (10mM), hydroxystearic acid (100mM), arachidonic acid $(100 \mathrm{mM})$, palmitic acid $(100 \mathrm{mM})$, and stearic acid $(100 \mathrm{mM})$ were made by dissolving each lipid in sterile $95 \%$ ethanol and stored at $-80^{\circ} \mathrm{C}$. Stock and dosage concentrations were determined by using similar concentrations as those used in other published studies within the literature ${ }^{107-109}$. During experimentation, SH-SY5Y cells were treated with each relevant lipid or the vehicle control (ethanol) dissolved into a media solution consisting of DMEM/F12 50/50 mix (Corning Cellgro) with L-glutamine, $15 \%$ freshly thawed Fetal Bovine serum (Atlanta Biologicals), 1\% Penicillin- 
Streptomycin solution (HycloneTM, 1000 units/mL Penicillin- $10000 \mu \mathrm{g} / \mathrm{mL}$

Streptomycin) for 4 hours followed by lysis.

Detergent-free Immunoprecipitation

FABP5-V5 tagged, overexpressing SH-SY5Y cells were plated onto a $100 \mathrm{~mm}$ cell culture dish and treated with retinoic acid for 7 days. Cells were then lysed using a freeze-thaw lysis technique. During lysis, cells were washed twice with phosphate buffer and frozen at $-80^{\circ} \mathrm{C}$ for 15 minutes in $120 \mu \mathrm{L}$ PBS. Cells were then thawed and scraped into microcentrifuge tubes. Following lysis, the cells were centrifuged at $15000 \mathrm{~g}$ for 10 minutes at $4^{\circ} \mathrm{C}$ and the supernatant was collected. The supernatant was incubated with either V5 antibody (D3H8Q, Cell Signaling Technology, catalog no. 13202S) or IgG isotype control antibody (Cell Signaling Technology, catalog no. 3900) for 1 hour at $4{ }^{\circ} \mathrm{C}$ on rotation. Following primary antibody incubation, ChIP grade protein G magnetic beads (Cell Signaling Technology, catalog no. 9006S) were added and the supernatant was rotated at $4{ }^{\circ} \mathrm{C}$. Then the magnetic bead complexes were pelleted and washed twice with PBS and once with ddH2O. Water was then removed, and magnetic bead pellets were resuspended in a 2:1 chloroform: methanol solution and sent for lipidomic analysis at The Emory University Lipidomics Core Facility.

Western Blotting

Western blots were run using the Life Technologies products. Before western blotting, cells were washed with PBS, lysed with RIPA lysis buffer containing a protease inhibitor cocktail and subsequently sonicated at $80 \%$ intensity for 7 seconds. Following sonication, cells were sonicated at $15000 \mathrm{~g}$ for 10 minutes at $4^{\circ} \mathrm{C}$, and the supernatant was then collected. Polyacrylamide Gel Electrophoresis was then performed on the protein 
extracts on either a $4-12 \%$ or $16 \%$ pre-cast gel, depending on the size of the protein assayed and the band separation required for proper analysis. Electrophoresed proteins were transferred onto a PVDF membrane using the iBlot2 Dry transfer device and transfer materials. The transfer was performed according to the manufacturer's instructions with the iBlot2 device.

Primary antibodies were used to detect LC3 (Novus Biologicals, catalog no. NB100-2220), FABP5 (Cell Signaling Technology, catalog no. 39926S), Phospho- S6 Ribosomal protein (Ser 235/236) (Cell Signaling Technology, catalog no.4858S), and $\beta$ actin (Thermo Fisher, catalog no. MA1-91399). Anti-rabbit (Cell Signaling Technology, catalog no. 7074) and anti-mouse (Cell Signaling Technology, catalog no. 7076) HRP secondary antibodies were used to probe western blot PVDF membranes after primary antibody incubation. Pierce ECL Western Blotting substrate (Thermo Scientific) was then used to develop membranes. Membranes were imaged on a Bio-Rad blot imager. Protein expression was analyzed by taking densitometry measurements on the images obtained, using ImageJ and Microsoft Excel.

\section{$\underline{\text { Lentiviral Production and Transduction }}$}

A Thermo Fisher Virapower kit was used to produce lentivirus. HEK 293T Human embryonic Kidney cells (ATCC) were transfected with the transgene-carrying plasmids, together with plasmids containing lentiviral packaging components, using lipofectamine 2000 (Life Technologies). Lentiviral packaging plasmids LP1 and LP2 as well as coat protein plasmid VSV and the desired transgene plasmid vector ( $7.2 \mu \mathrm{g}$ of each) were incubated with Lipofectamine 2000 in Opti-MEM media (Gibco) for 20 
minutes at room temperature. The mixture was then added dropwise to flasks containing $293 \mathrm{~T}$ cells in fresh media. The next day, media was replaced with fresh media. The following day, the supernatant containing full assembled, active lentivirus was collected and filtered through $400 \mathrm{~nm}$ pore filters. Filtered supernatant media was aliquoted as needed and stored at $-80^{\circ} \mathrm{C}$ until ready for use. For transduction into target cells, aliquoted virus-containing media was added directly to the media bathing the target cells in their flasks. The following day, media was replaced with fresh media. Puromycinbased selection for shRNA constructs was initiated three days after viral addition and maintained for the duration of the experiment.

\section{LC3B-GFP-Mcherry Puncta Assessment}

SH-SY5Y cells were plated into 24-well plates containing poly D-lysine coated coverslips. Cells were transduced with lentiviral stocks containing the FUW McherryGFP-LC3 plasmid obtained as a gift from Anne Brunet (Addgene, plasmid \# 110060) ${ }^{110}$. The following day virus-containing media was replaced with fresh media. 48 hours after initial transduction cells were differentiated into neurons with $10 \mu \mathrm{M}$ RA for 7 days. Following the differentiation period, cells were treated with relevant lipids for 4 hours. Treated cells were then fixed with 4\% paraformaldehyde (in PBS) for 23 minutes on ice. The glass coverslips with attached cells were then washed with PBS and incubated in 1:10000 Hoechst dye in PBS for 5 minutes on ice and in the dark and washed again. Finally, the coverslips were mounted on glass slides for fluorescent imaging using Fluoromount G. Glass slides were imaged on the EVOS M5000 imaging system (Thermo Fisher Scientific) at 60x objective magnification with the automated z-stack function. Red only puncta and green only puncta were counted with ImageJ on each image 
produced and a value for flux was determined as the ratio of red only puncta to total puncta for each image. This processing was automated via macro to create objective quantification of puncta.

\section{$\underline{\text { RNA Extraction }}$}

SH-SY5Y cells were plated onto 6-well plates and treated with either vehicle control (ethanol), 5OE (40 $\mu \mathrm{M})$, SA (400 $\mu \mathrm{M})$ or HSA (400 $\mu \mathrm{M})$ for 6 hours. Following treatment, cells were lysed using the materials included in the Qiagen RNeasy RNA extraction Minikit (catalog no, 74104). Total RNA was extracted with included materials according to the manufacturer's protocol. RNA concentrations and purity were measured using a spectrophotometer (Implen Pearl nanophotometer).

\section{RNA Seq}

Extracted RNA was sequenced and analyzed by Novogene. Briefly, RNA was poly-A captured (rRNA depleted), RNA fragmented and cDNA synthesized. Sequencing was performed using an Illumina NovaSeq 6000 system. Analysis was performed for paired-end 150 bp reads with $\geq 20$ million reads per sample. A differential gene expression analysis was performed and KEGG and Reactome annotated pathways enrichment determined.

\section{Results}

FABP5 is Expressed by Dopaminergic Neurons in the Substantia Nigra

In situ hybridization data, provided by the Allen Brain Institute, transcriptomics previously reported ${ }^{100}$ and our immunohistochemical analysis (Figure 2.1) indicates that FABP5 mRNA and protein expression is enriched in dopaminergic neurons within the substantial nigra of mouse brain, a region that possesses dopaminergic neurons whose 
loss results in the hallmark motor features of PD. As a result, we chose to utilize a human neuroblatoma cell line (SH-SY5Y) for this investigation that can be differentiated into post-mitotic dopaminergic neuron-like cells. These cells are widely employed by PD researchers and, due to their ready production of post-mitotic neuron-like cells, they are also used broadly across neuroscience disciplines including AD, ALS and HD research 111.

A

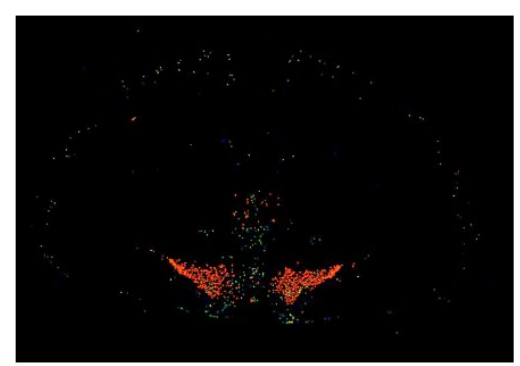

C

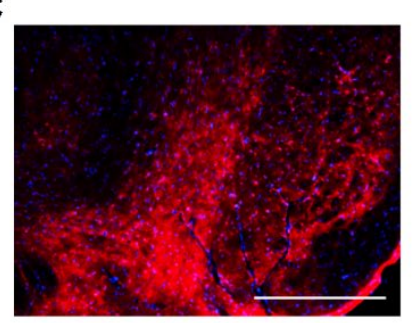

D
B
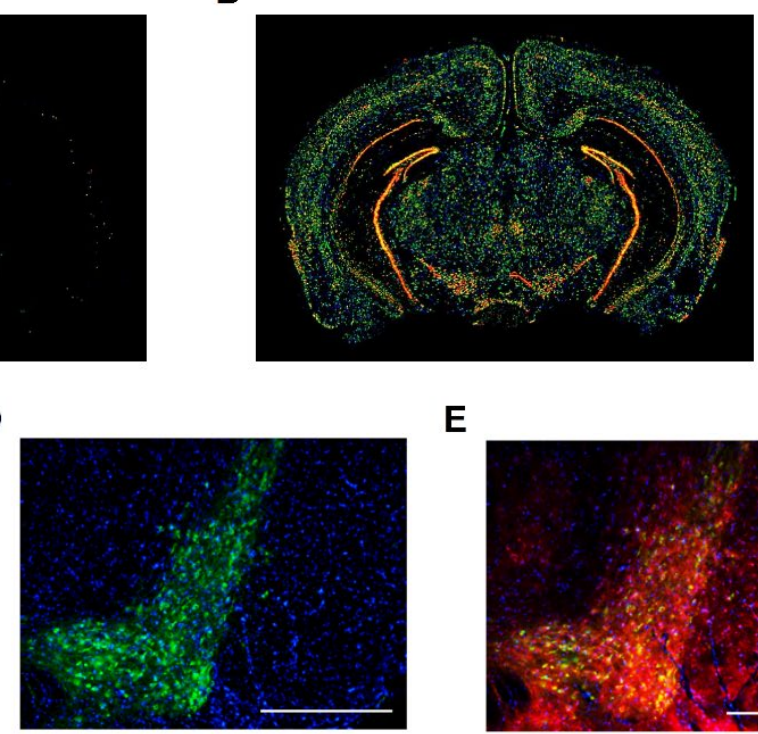

E

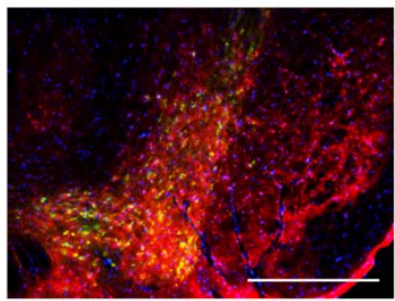

Figure 2.1. FABP5 is expressed by nigral dopaminergic neurons. In situ hybridization for Tyrosine hydroxylase (A) and Fabp5 (B) mRNA expression [Mouse

Brain Atlas, Allen Brain Institute]. Immunohistochemistry was performed for FABP5 (C) and Tyrosine Hydroxylase (D) in adult mouse brain substantia nigra with nuclei labeled using DAPI and merged image shown (E). Scale bar $=400 \mu \mathrm{m}$.

\section{FABP5 Regulates Autophagy}

To address whether FABP5 affects autophagy, we engineered SH-SY5Y cells to stably express scrambled or FABP5 shRNA. A pure population of transgenic cells was then obtained by selection with puromycin. SH-SY5Y cells were then differentiated into post-mitotic dopaminergic neuron-like cells with 7 days of $10 \mu \mathrm{M}$ of all-trans retinoic acid (RA) supplemented to growth media as previously described ${ }^{111,112}$. Autophagic flux 
was determined by comparing the autophagosome marker LC3B-II in untreated versus bafilomycin A1 treated cultures (Figure 2.2A, B) ${ }^{113}$. We also monitored autophagic flux by quantifying autophagosome/lysosome ratios observed in mCherry-GFP-LC3B transgene-expressing cells (Figure 2.2C, D) as previously described ${ }^{113,114}$. Our data indicates that FABP5 knockdown results in impaired autophagy. In addition, we found that neither FABP5 knockdown nor overexpression affected SH-SY5Y differentiation (Figure 2.3), indicating that differences in these cells were not due to altered RA signaling or differentiation status.

A

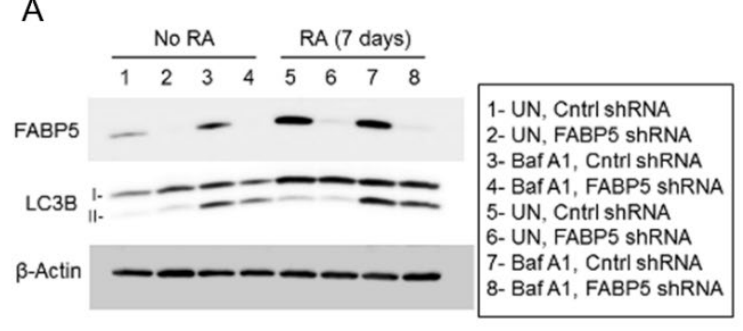

C

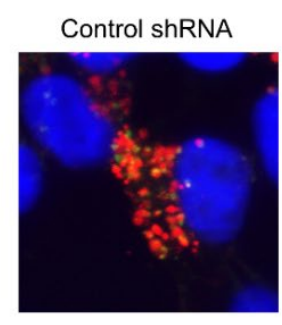

FABP5 shRNA

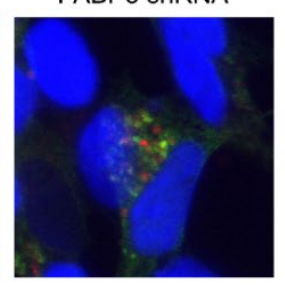

B

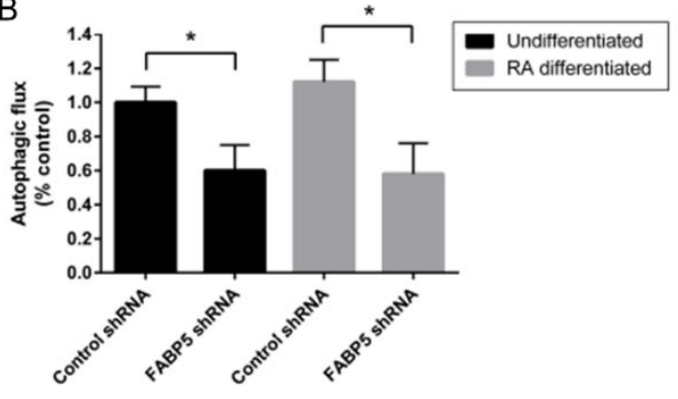

D

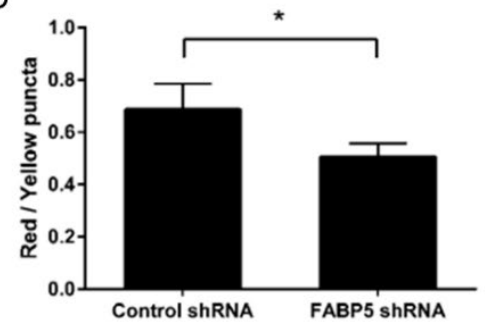

Figure 2.2. FABP5 regulates autophagy. (A) Undifferentiated or RAdifferentiated SH-SY5Y cells were transduced with lentivirus containing scrambled (Cntrl) or FABP5 shRNA constructs and LC3B-IIexpression evaluated by western blot and (B) autophagic flux determined. (C) Representative images for mCherrygfp-LC3B that was transduced by lentivirus into RA-differentiated control or FABP5 knockdown as described in A. and then autophagosome (yellow) and lysosomes (red) quantified (D). * $\mathbf{p}<0.05$, error bars $=$ SEM. 


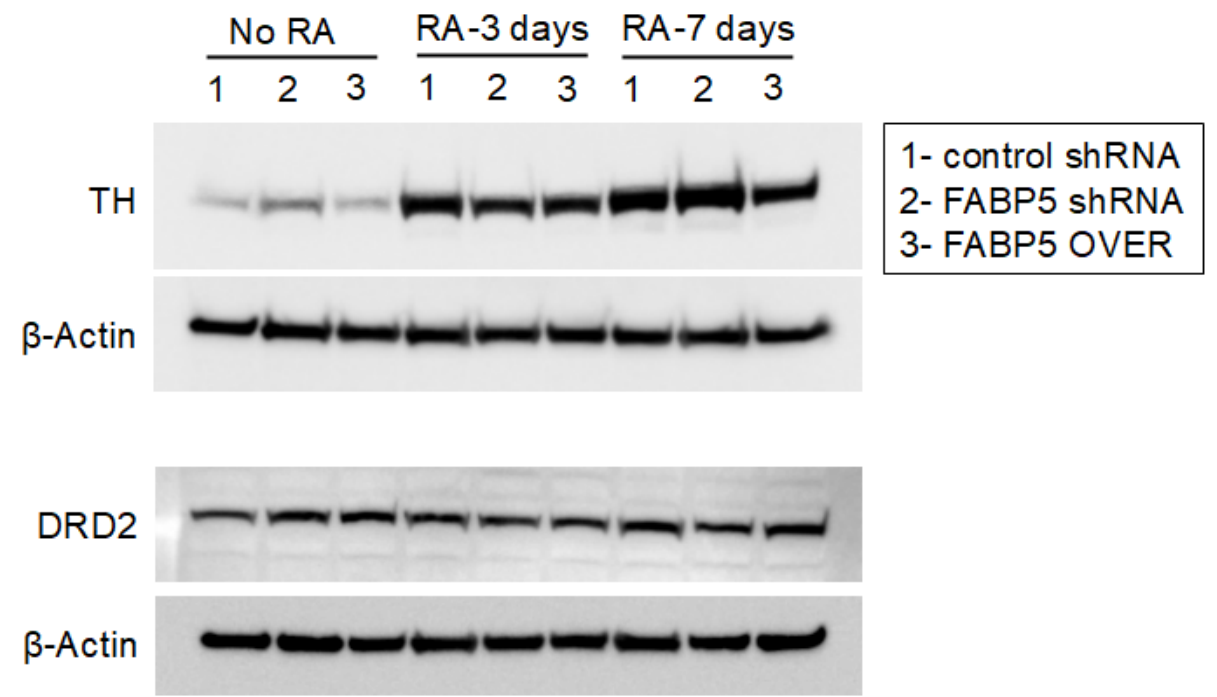

Figure 2.3. FABP5 does not affect SH-SY5Y differentiation by RA. SH-SY5Y cells were transduced with lentivirus containing a scrambled (control) shRNA, FABP5 shRNA or an FABP5 overexpression (OVER) construct. Cells were selected for transgene expression using puromycin and treated with all-trans RA (10 $\mu \mathrm{M})$ for the indicated times and Tyrosine Hydroxylase (TH) and Dopamine receptor $\mathrm{D}_{2}$ (DRD2) protein levels were assessed by western blot. 3 biological replicates are shown.

5OE, AA, HSA, SA and PA Interact with FABP5

Having established an autophagy regulating function for FABP5, we next employed FABP5 as a bait protein to identify lipid interactors. We created an SH-SY5Y cell line using a lentivirus that stably expressed FABP5 with a V5 epitope tag. A detergent-free immunoprecipitation protocol was developed to co-purify FABP5 and associated lipids (Figure 2.4A). Cells were RA-differentiated for 7 days and then treated with media containing fresh FBS for 1 hour. FABP5-lipid complexes were then purified and sent to the Emory University lipidomics core for analysis. Six lipids (one unidentifiable by mass) were found to exhibit FABP5 binding (Figure 2.4B). Arachidonic acid (AA) was identified in this group and is well-studied as a precursor for a diverse set of metabolites, including 5-Oxo-eicosatetraenoic acid (5OE), another FABP5 interactor found in this analysis. Long 
chain fatty acids were also identified that include hydroxystearic acid (HSA), stearic acid (SA) and palmitic acid (PA).

A
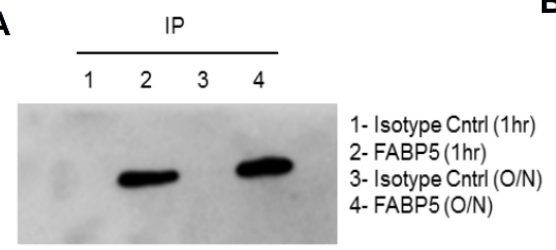

B

\begin{tabular}{|l|l|c|l|}
\hline Lipids & $\mathbf{m z}$ & V5/Control ratio & p-value \\
\hline Undecylic acid, ISTD & $\mathbf{2 7 7}$ & 1.00 & N/A \\
\hline Palmitic acid & 347 & 1.21 & 0.0036 \\
\hline Stearic acid & 375 & 1.16 & 0.0025 \\
\hline 12-Hydroxystearic acid & 391 & 1.31 & 0.0024 \\
\hline Arachidonic acid & 395 & 1.15 & 0.0009 \\
\hline 5-Oxo-ETE & 409 & 1.36 & 0.0017 \\
\hline Unknown & 772 & 2.16 & 0.0023 \\
\hline
\end{tabular}

Figure 2.4. FABP5 lipidomic interactome.(A) FABP5 was successfully immunoprecipitated (IP) with a detergent-free method using lysate obtained from RA-differentiated SH-SY5Y cells that stably express a recombinant FABP5-V5. Immunoprecipitations (V5 epitope tag-specific or antibody isotype control) were performed for $1 \mathrm{hr}$ or overnight $(\mathrm{O} / \mathrm{N})$ and western blotting done using an FABP5 antibody. (B) Samples obtained by 1 hr FABP5-V5 IP (V5) or control antibody IP

(Control) from 3 independent biological replicates were processed for lipid extraction and analyzed by QTRAP 5500 mass spectrometer via Exion LC AC autosampler. Six lipids were identified to have significant interaction with FABP5.

\section{Autophagy Inhibition by 5OE, HSA and SA but not AA or PA}

We next examined whether each of these five candidates affected autophagy in differentiated SH-SY5Y cells. We found that 5OE, HSA and SA, but not AA or PA, exhibited potent dose-dependent anti-autophagic activity at concentrations reported in human serum ${ }^{115-117}$ as well as in other systems (Figures $\left.2.5,2.6\right)^{118-120}$. Interestingly, AA shows an altered lysosome/autophagosome ratio following quantification of mCherryGFP-LC3B puncta (Figure 2.6) indicative of autophagy inhibition. However, an examination of LC3-II levels following bafilomycin treatment (Figure 2.5C., D) has 

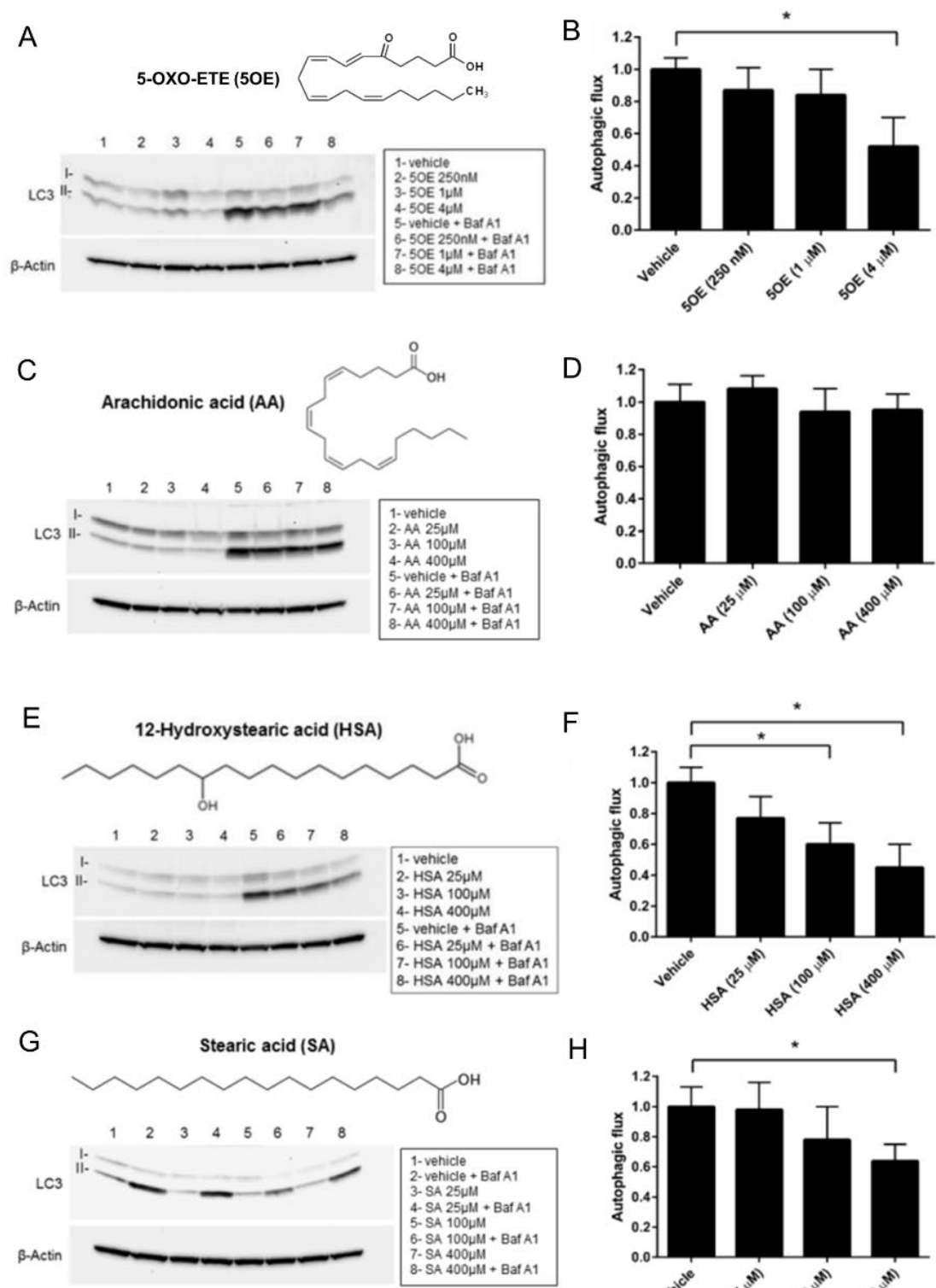

$\mathrm{H}$
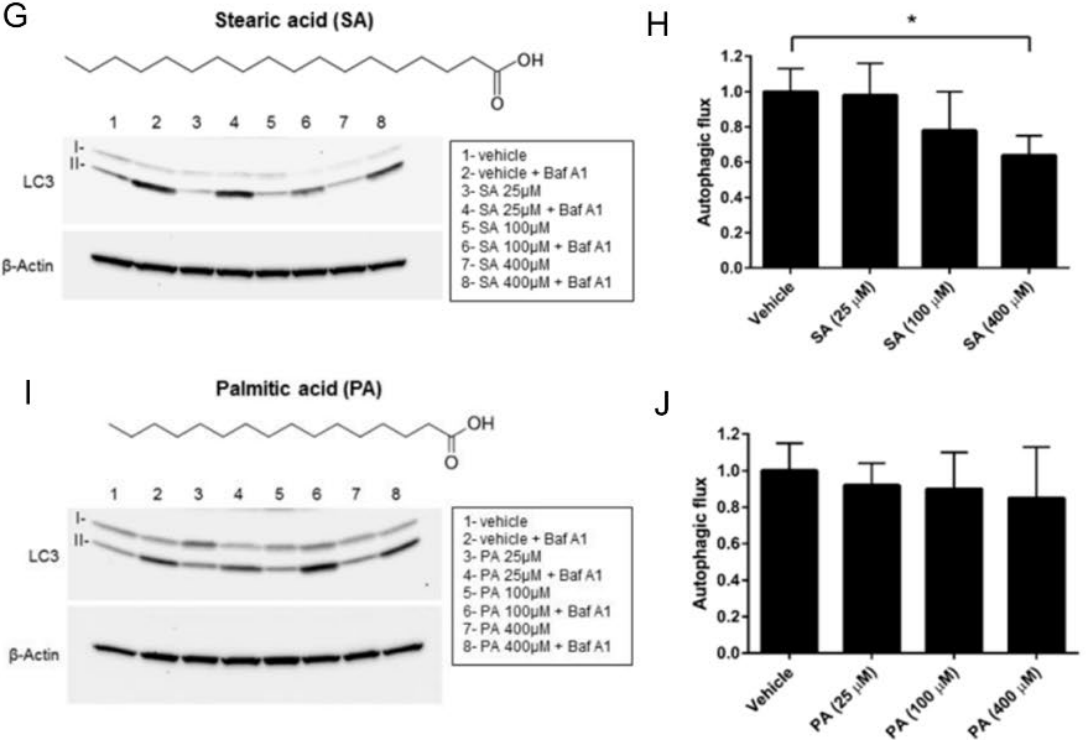

Figure 2.5. Effect of candidate lipids on LC3B-II autophagic flux. (A,C,E,G,I) Increasing amounts of lipid or vehicle (ethanol) were added to RA-differentiated SH-SY5Y cells for $4 \mathrm{~h}$ in the presence or absence of bafilomycin A1 (Baf A1) and western blotting performed and autophagic flux was determined (B,D,F,H,J) from 3 biological replicates. ${ }^{*} \mathbf{p}<\mathbf{0 . 0 5}$, error bars $=$ SEM. 
revealed no effect on autophagic flux. We observed that at 4 hours of treatment large numbers of cells were detached in the two higher doses of AA, suggesting toxicity. This was confirmed at 24 hours of treatment (data not shown). Given many shared mechanisms between autophagy and apoptosis, these results suggest AA induces death rather than autophagy ${ }^{121}$.

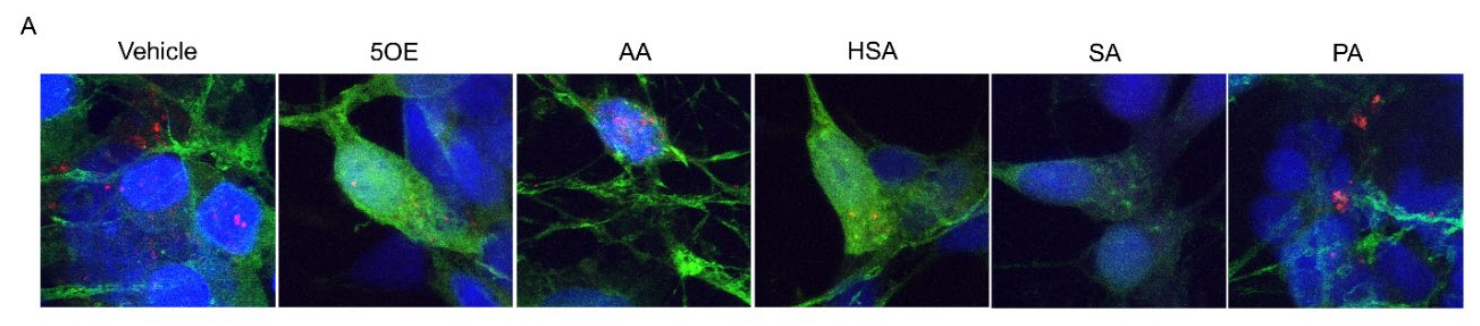

B

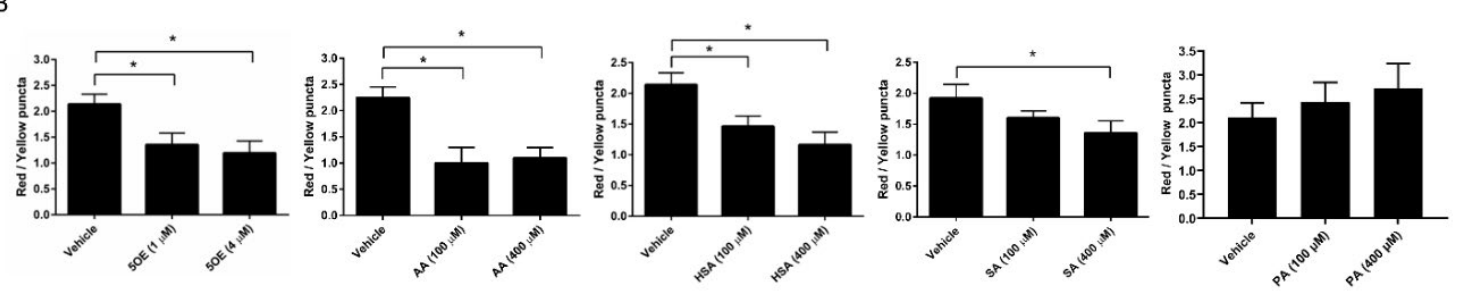

Figure 2.6. Effect of candidate lipids on LC3B puncta autophagic flux. Lentivirus was used to transduce RA-differentiated SH-SY5Y cells with mCherry-gfp-LC3B. Seven days later, cells were treated with ethanol (vehicle) or lipid at the highest two does for $4 \mathrm{~h}$. Representative images (A) are shown for the highest lipid dose and autophagosomes (yellow) and lysosomes (red) were then quantified (B). *p $<0.05$, error bars $=$ SEM.

Treatment with 5OE, HSA or SA Enhances mTORC1 Pathway Signaling

The mammalian target of rapamycin complex 1 (mTORC1) complex is a master regulator of autophagy via integration of numerous and diverse signaling pathways ${ }^{122}$. To further validate autophagic flux data for 5OE, HSA and SA, we assessed their activation of mTORC1. We tested whether candidate lipids could regulate mTORC1 activation by treating differentiated SH-SY5Y cells with autophagy inhibiting doses of 5OE, HSA or SA and measured the activation of a canonical mTORC1 substrate, S6, by 
western blot (Figure 2.7). An increase in the phosphorylation of S6 at mTORC1associated residues (S240/S244) was observed following 5OE and HSA at 15 min (Figure 2.7C) and SA at $1 \mathrm{hr}$ (Figure 2.7D) compared to vehicle. Activation of S6 was largely unchanged at $1 \mathrm{~h}$ and $2 \mathrm{~h}$ post-treatment with the exception of SA. No effect was observed by PA treatment. It should be noted that lipids were gently but thoroughly mixed with fresh media containing $15 \%$ FBS by tube inversion and added to cells at time zero. FBS possesses lipid carrier proteins (e.g., albumin) to facilitate proper shuttling of lipids to cultured cells in a physiological manner. Also note, that the initial robust increase in pS6 (at $30 \mathrm{~min}$ ) in vehicle-treated cells is likely due to enhanced mTOR signaling resulting from this addition of fresh FBS-containing media. However, 5OE, HSA and SA alter this dynamic. This data supports an autophagy-inhibiting role for 5OE, HSA and SA and suggests that this could involve an mTORC1-mediated mechanism. 

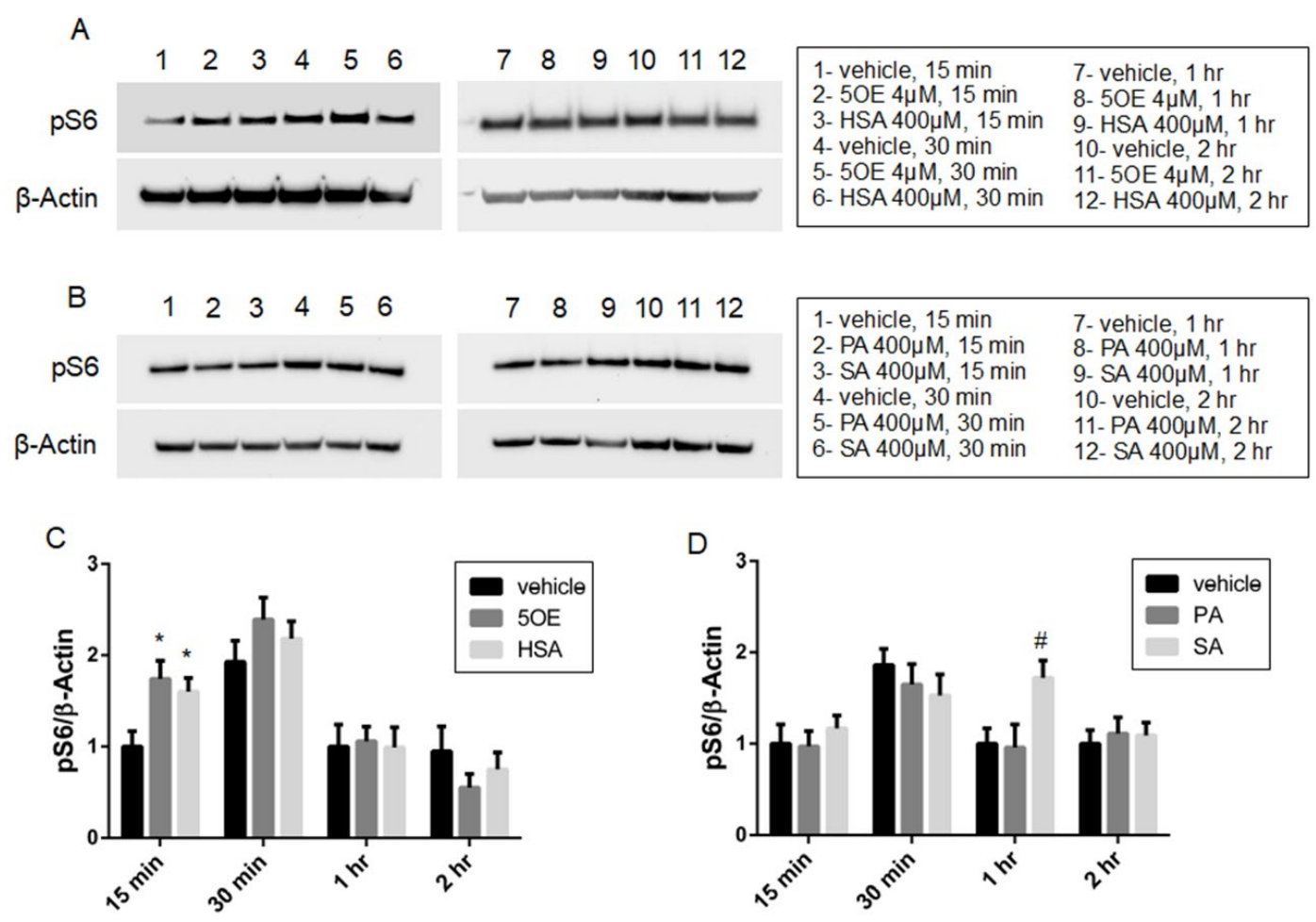

Figure 2.7. mTOR pathway activation by 5OE, HSA, SA, and PA. SH-SY5Y cells were differentiated with RA and then subjected to ethanol (vehicle), 5OE, HSA, SA or PA for the indicated concentrations and times in freshly prepared culture media.

Western blotting (A, B) was then performed for $\mathrm{pS6}$ (Ser240/244) following treatment at the indicated times. Densitometry was assessed for $\mathrm{pS6}$ /B-Actin for each condition and time point normalized to vehicle for each time point $(C, D) .{ }^{*} p<$ 0.05 , error bars $=$ SEM versus vehicle group at each time point.

\section{$\underline{\text { 5OE, HSA and SA Do Not Induce Cell Death }}$}

Autophagic mechanisms can share features with apoptosis ${ }^{123}$. Therefore, we examined whether the lipids, 5OE, HSA or SA, which exhibited an autophagy-regulating profile in both autophagic flux assay (Figures 2.5 and 2.6) induced death in differentiated SH-SY5Y cells. These lipids did not exhibit toxicity after 24 hours of treatment (Figure 2.8) indicating that apoptosis is not confounding autophagic flux data. Interestingly, despite structural similarity, AA showed robust toxicity at 100uM and 400uM doses (data not shown) suggesting divergent effects with $5 \mathrm{OE}$. 

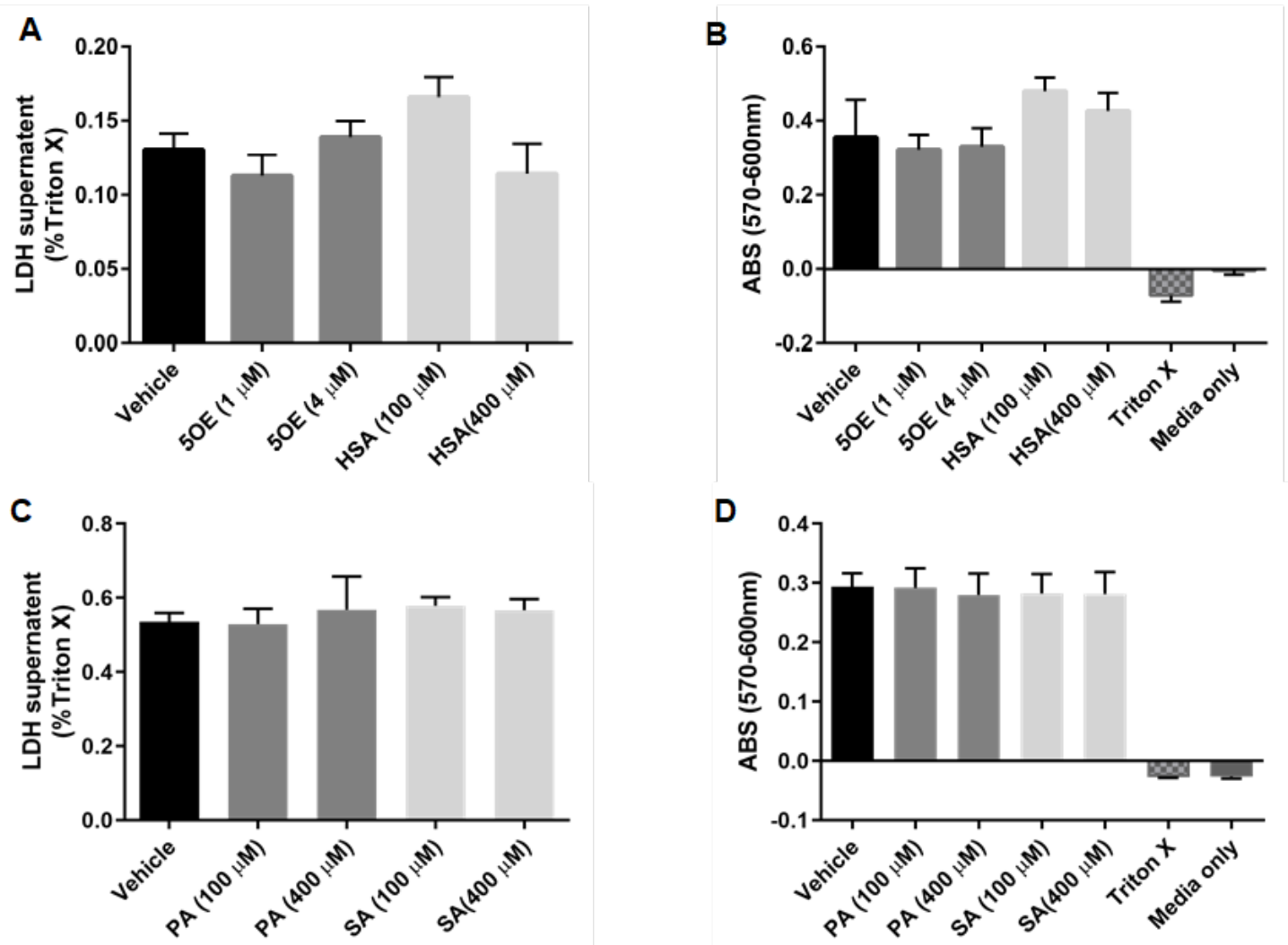

Figure 2.8. 5OE, HSA, SA and PA do not induce cell death. RA-differentiated SH-SY5Y cells were treated with ethanol (vehicle), 5OE, HSA, SA or PA as shown in normal growth media. After $24 \mathrm{~h}$, cell death was measured by (A, C) LDH released into the culture media as well as $y$ the $(B, D)$ resazurin assay. ${ }^{*} p<0.05$, error bars $=$ SEM.

Transcriptomic Alteration of the PPAR Pathway by HSA

We next sought to gain insight into the molecular mechanism of action for autophagy-inhibiting lipid candidates. RNA Seq (random primed with 44M-60M reads/sample) was performed on RA-differentiated SH-SY5Y cells treated with vehicle (ethanol) or lipid (5OE: $4 \mu \mathrm{M}$, HSA: $400 \mu \mathrm{M}$, SA: $400 \mu \mathrm{M}$ ) for 6 hours. Unbiased analysis for transcript enrichment for KEGG and Reactome annotated pathways was performed (Figure 2.9). Large numbers of differential transcripts for both HSA and SAtreated cells were identified (Figure 2.9H, I). However, only a very modest number of differential transcripts in the 5OE group was found (Figure 2.9G) which reduces the 
pathway associations as evidenced by only one significant hit (amyotrophic lateral sclerosis). For this reason, we also listed the top associated pathways that were not significant. Interestingly, the mTOR signaling pathway was such a hit. Both HSA and SA exhibited associations with cell cycle regulation, fatty acid metabolism and peroxisome proliferator-activated receptor (PPARA) signaling. 

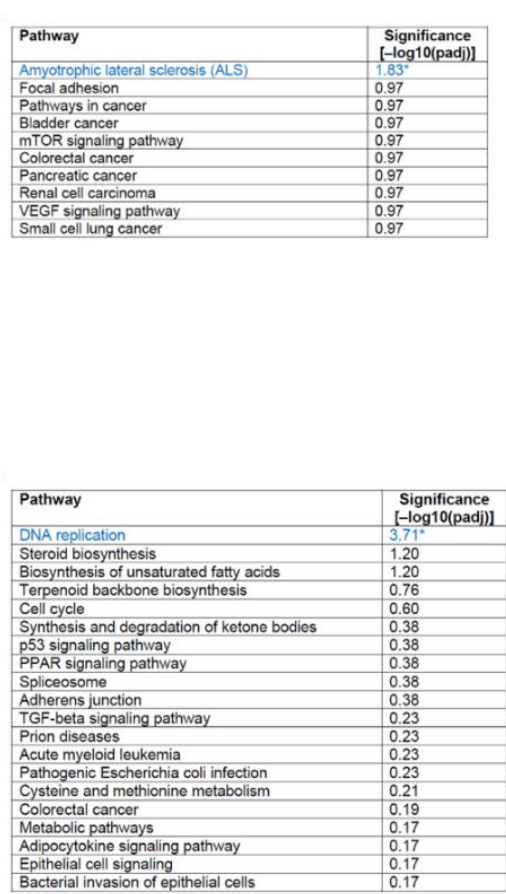

E

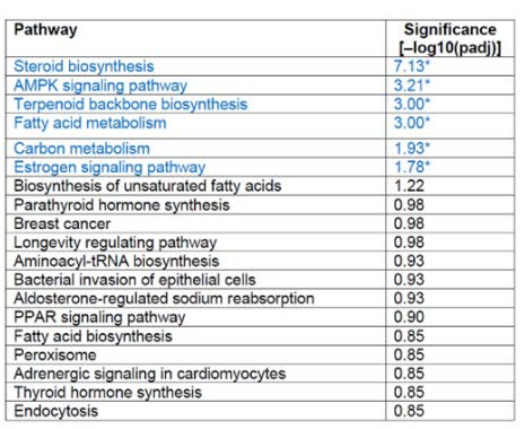

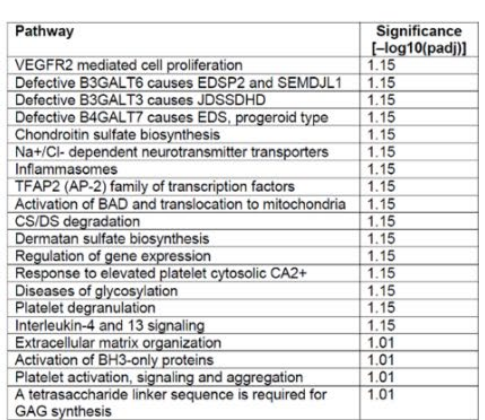

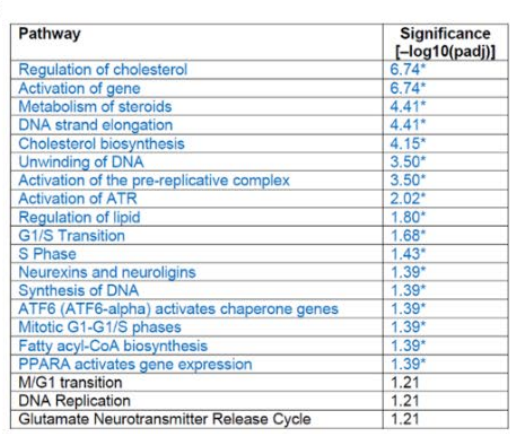

$\mathbf{F}$
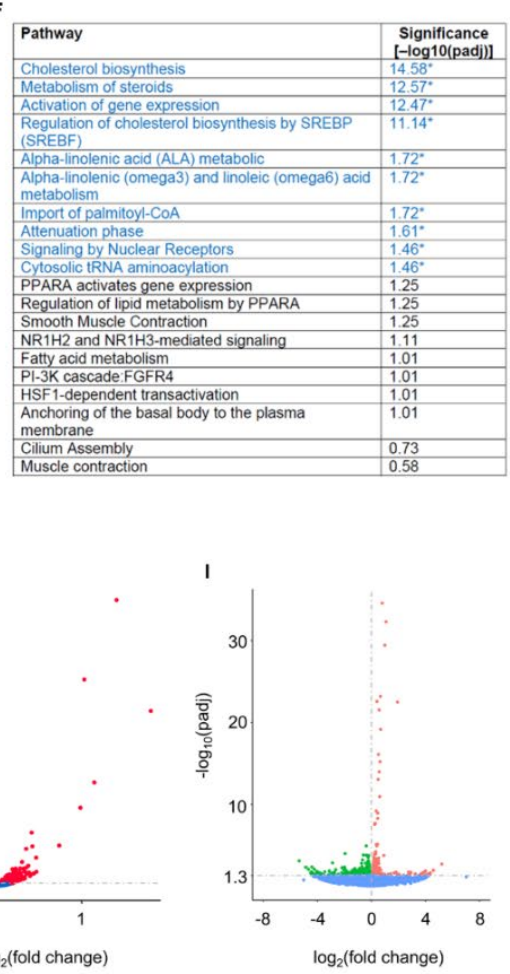

Figure 2.9. RNA Seq analysis of lipid treatment.KEGG annotated pathways for RA-differentiated SH-SY5Y cells treated with (A) $4 \mu \mathrm{M}$ 5OE, (C) $400 \mu \mathrm{M}$ HSA and (E) $400 \mu \mathrm{M}$ SA for $6 \mathrm{~h}$. (B, D, F) Reactome pathways are also shown for each treatment. *significance versus vehicle treatment group; 3 biological replicates. Volcano plots for (G) $4 \mu \mathrm{M}$ 5OE, (F) $400 \mu \mathrm{M}$ HSA and (G) $400 \mu \mathrm{M}$ SA are shown $($ red dots $=$ up regulated genes, green dots $=$ down regulated genes). 


\section{Discussion}

Despite intense efforts to harness autophagy for therapeutic benefit, very few clinical applications have been realized ${ }^{124}$. Consequently, the strong association between autophagic dysfunction and neurodegenerative diseases like PD has spurred investigation into more diverse targets for autophagy regulation. This rationale prompted us to devise a lipidomic screen for identification of autophagy-controlling lipids. Our study has identified three lipids, 5OE, HSA and SA, with potent anti-autophagic activity when applied to cultured dopaminergic cells at physiological concentrations found in human serum. In addition, this work has uncovered FABP5 as a regulator of autophagy, likely through the activity of lipid cargo.

5OE and AA are both well-established signaling lipids that were significant hits in our FABP5 interactome screen. AA also serves as the precursor for a diverse set of bioactive lipid signaling molecules making it of great interest in human physiology and disease ${ }^{125}$. Nevertheless, since we do not observe a direct effect on autophagy in our system, we did not explore AA biology further. We do note, however, that AA is an interactor of FABP5 and a precursor for 5OE, which provides additional evidence for a physiological FABP5-5OE interaction. 5OE can be produced and released locally or as a precursor metabolite (5-HETE) released and metabolized by the target cell into 5OE (via transcellular biosynthesis) ${ }^{126}$. 5OE has been well-studied for its role as an inflammatory cytokine that can affect a wide range of leukocytes ${ }^{127}$. However, 5OE is reported to exhibit the greatest potency on eosinophils as both a chemotactic agent and a stimulant, suggesting an anti-parasitic or allergic response role ${ }^{127,128}$. Interestingly, previous work has uncovered another allergy response pathway expressed by dopaminergic neurons that 
sensitizes these cells to death ${ }^{129}$. This raises the question of whether dopaminergic neurons are susceptible to cell stress induced by allergic response signaling. A possible explanation for heightened expression of FABP5 in dopaminergic neurons of the substantia nigra (Figure 2.1) is to sequester 5OE due to enhanced sensitivity toward this mode of allergic response signaling.

Our data supports a direct role for 5OE upon dopaminergic neurons, which is consistent with reports of other proinflammatory cytokines (e.g., IL-6, TNF-alpha) ${ }^{130}$. While there are reports of an oxoeicosanoid receptor 1 (OXER1) $)^{131}$ that can interact with $5 \mathrm{OE}$ as well as other eicosanoids, leukotrienes and $5 \mathrm{OE}$ metabolites ${ }^{132}$, it is likely not responsible for autophagy inhibition by 5OE that we observe. This is because OXER1 is a plasma membrane-bound G-protein-coupled receptor, while our isolated FABP5-5OE complexes reside within the cytoplasm (mild, detergent-free immunoprecipitation). We have also observed that FABP5 suppression by shRNA inhibits autophagy, indicating a cytoplasmic role for $5 \mathrm{OE}$.

HSA and SA are long-chain fatty acids with an 18-carbon backbone that were also identified by our FABP5 lipidomic interactome screen. SA is a lipid commonly found at high levels in a large number of food products, including grains, milk, meat, and dairy. Thus, all individuals have some exposure to this compound. There are no reports of direct toxicity from SA. However, mounting evidence implicates this dietary lipid as a potential regulator of cell cycle and tumorigenesis, albeit with conflicting reports ${ }^{108,133}$. Metabolic derangement has also been noted in cells and rodents following SA exposure 134. Further study of SA is likely warranted given the ubiquitous presence of this lipid in food products and the newly found association with autophagy inhibition reported here. 
The addition of a single hydroxyl group to SA at the 12th carbon results in the creation of HSA. While HSA is not a natural food compound, it is found in considerable amounts in a large number of consumer products and can be produced by microbiota from oleic acid and could provide a natural source of unknown dosage in animals ${ }^{135}$. The synthetic addition of the hydroxyl group to SA provides favorable handling characteristics (waxiness), leading to the wide adoption of HSA in cosmetic products (e.g., shampoo, underarm deodorants, lipsticks and more). Similar to SA, the study of HSA has suggested its potential involvement in regulating tumorigenesis ${ }^{136,137}$. In line with this observation, we found a strong association with cell cycle alteration and oncogenic pathways in our RNA Seq analysis of HSA-treated cells (Figure 2.9). Interestingly, both SA and HSA exhibited strong associations with PPAR signaling pathways for KEGG and Reactome annotated pathways in our analysis. PPAR alpha and delta are known to propagate lipid signaling through FABP5 interaction ${ }^{138,139}$ suggesting that this is a pathway of interest for further study of autophagy inhibition.

In summary, we report that FABP5 regulates autophagy in dopaminergic cells. Our subsequent lipidomic interactome screen identified five FABP5 interactors. Of these interactors, 5OE, HSA and SA were confirmed to inhibit autophagy in dopaminergic neurons. RNA Seq analysis for 5OE yielded few clues to mechanism of action due to low differential transcript expression versus control. Conversely, transcriptomic assessment for both HSA and SA indicates a strong association with altered PPAR signaling which provides an intriguing target for further investigation into HSA/SAFABP5-mediated autophagy inhibition. Overall, these results indicate that identification 
of lipids that modulate autophagy could be a fruitful avenue for developing novel therapeutics. 


\section{References}

1. Mizushima, N. (2007). Autophagy: process and function. Genes \& Development, 21(22), 2861-2873. https://doi.org/10.1101/gad.1599207

2. Noda, T., \& Ohsumi, Y. (1998). Tor, a phosphatidylinositol kinase homologue, controls autophagy in yeast. Journal of Biological Chemistry, 273(7), 3963-3966. https://doi.org/10.1074/jbc.273.7.3963

3. Jung, C. H., Jun, C. B., Ro, S.-H., Kim, Y.-M., Otto, N. M., Cao, J., ... Kim, D.H. (2009). ULK-Atg13-FIP200 Complexes Mediate mTOR Signaling to the Autophagy Machinery. Molecular Biology of the Cell, 20(7), 1992-2003. https://doi.org/10.1091/mbc.e08-12-1249

4. Zhong, Y., Wang, Q. J., Li, X., Yan, Y., Backer, J. M., Chait, B. T., ... Yue, Z. (2009). Distinct regulation of autophagic activity by Atg14L and Rubicon associated with Beclin 1-phosphatidylinositol-3-kinase complex. Nature Cell Biology, 11(4), 468-476. https://doi.org/10.1038/ncb1854

5. Axe, E. L., Walker, S. A., Manifava, M., Chandra, P., Roderick, H. L., Habermann, A., ... Ktistakis, N. T. (2008). Autophagosome formation from membrane compartments enriched in phosphatidylinositol 3-phosphate and dynamically connected to the endoplasmic reticulum. Journal of Cell Biology, 182(4), 685-701. https://doi.org/10.1083/jcb.200803137

6. Kabeya, Y., Mizushima, N., Yamamoto, A., Oshitani-Okamoto, S., Ohsumi, Y., \& Yoshimori, T. (2004). LC3, GABARAP and GATE16 localize to autophagosomal membrane depending on form-II formation. Journal of Cell Science, 117(13), 2805-2812. https://doi.org/10.1242/jcs.01131

7. Hanada, T., Noda, N. N., Satomi, Y., Ichimura, Y., Fujioka, Y., Takao, T., ... Ohsumi, Y. (2007). The Atg12-Atg5 conjugate has a novel E3-like activity for protein lipidation in autophagy. Journal of Biological Chemistry, 282(52), 3729837302. https://doi.org/10.1074/jbc.C700195200 
8. Fujita, N., Itoh, T., Omori, H., Fukuda, M., Noda, T., \& Yoshimori, T. (2008). The Atg16L Complex Specifies the Site of LC3 Lipidation for Membrane Biogenesis in Autophagy. Molecular Biology of the Cell, 19(5), 2092-2100. https://doi.org/10.1091/mbc.e07-12-1257

9. Pankiv, S., Clausen, T. H., Lamark, T., Brech, A., Bruun, J.-A., Outzen, H., ... Johansen, T. (2007). p62/SQSTM1 Binds Directly to ATG8/LC3 to Facilitate Degradation of Ubiquitinated Protein Aggregates by Autophagy. Journal of Biological Chemistry, 282(33), 24131-24145. https://doi.org/10.1074/jbc.M702824200

10. Cebollero, E., Van Der Vaart, A., Zhao, M., Rieter, E., Klionsky, D. J., Helms, J. B., \& Reggiori, F. (2012). Phosphatidylinositol-3-phosphate clearance plays a key role in autophagosome completion. Current Biology, 22(17), 1545-1553. https://doi.org/10.1016/j.cub.2012.06.029

11. Ishihara, N., Hamasaki, M., Yokota, S., Suzuki, K., Kamada, Y., Kihara, A., ... Ohsumi, Y. (2001). Autophagosome requires specific early sec proteins for its formation and NSF/SNARE for vacuolar fusion. Molecular Biology of the Cell, 12(11), 3690-3702. https://doi.org/10.1091/mbc.12.11.3690

12. Gutierrez, M. G., Munafó, D. B., Berón, W., \& Colombo, M. I. (2004). Rab7 is required for the normal progression of the autophagic pathway in mammalian cells. Journal of Cell Science, 117(13), 2687-2697. https://doi.org/10.1242/jcs.01114

13. Tanaka, Y., Guhde, G., Suter, A., Eskelinen, E. L., Hartmann, D., LüllmannRauch, R., ... Saftig, P. (2000). Accumulation of autophagic vacuoles and cardiomyopathy LAMP-2-deficient mice. Nature, 406(6798), 902-906. https://doi.org/10.1038/35022595

14. Yang, Z., Huang, J., Geng, J., Nair, U., \& Klionsky, D. J. (2006). Atg22 Recycles Amino Acids to Link the Degradative and Recycling Functions of Autophagy. Molecular Biology of the Cell, 17(12), 5094-5104. https://doi.org/10.1091/mbc.e06-06-0479 
15. Liang, X. H., Jackson, S., Seaman, M., Brown, K., Kempkes, B., Hibshoosh, H., \& Levine, B. (1999). Induction of autophagy and inhibition of tumorigenesis by beclin 1. Nature, 402(6762), 672-676. https://doi.org/10.1038/45257

16. Cuervo, A. M. (2004). Autophagy: In sickness and in health. Trends in Cell Biology, 14(2), 70-77. https://doi.org/10.1016/j.tcb.2003.12.002

17. Paglin, S., Hollister, T., Delohery, T., Hackett, N., McMahill, M., Sphicas, E., ... Yahalom, J. (2001). A novel response of cancer cells to radiation involves autophagy and formation of acidic vesicles. Cancer Research, 61(2), 439-444

18. Meijer, A. J., \& Codogno, P. (2004). Regulation and role of autophagy in mammalian cells. International Journal of Biochemistry and Cell Biology, 36(12), 2445-2462. https://doi.org/10.1016/j.biocel.2004.02.002

19. Stambolic, V., Suzuki, A., De la Pompa, J. L., Brothers, G. M., Mirtsos, C., Sasaki, T., ... Mak, T. W. (1998). Negative regulation of PKB/Akt-dependent cell survival by the tumor suppressor PTEN. Cell, 95(1), 29-39. https://doi.org/10.1016/S0092-8674(00)81780-8

20. Yue, Z., Jin, S., Yang, C., Levine, A. J., \& Heintz, N. (2003). Beclin 1, an autophagy gene essential for early embryonic development, is a haploinsufficient tumor suppressor. Proceedings of the National Academy of Sciences of the United States of America, 100(25), 15077-15082. https://doi.org/10.1073/pnas.2436255100

21. Qu, X. (2003). Promotion of tumorigenesis by heterozygous disruption of the beclin 1 autophagy gene. Journal of Clinical Investigation, 112(12), 1809-1820. https://doi.org/10.1172/JCI200320039

22. Nishino, I. (2006). Autophagic Vacuolar Myopathy. Seminars in Pediatric Neurology, 13(2), 90-95. https://doi.org/10.1016/j.spen.2006.06.004

23. Nishino, I., Fu, J., Tanji, K., Yamada, T., Shimojo, S., Koori, T., ... Hirano, M. (2000). Primary LAMP-2 deficiency causes X-linked vacoular cardiomyopathy and myopathy (Danon disease). Nature, 406(6798), 906-910. https://doi.org/10.1038/35022604 
24. Shinatani, T., \& Klionsky, D. J. (2004). Autophagy in Health and Disease: A Double-Edged Sword. Science, 306(5698), 990-995. https://doi.org/10.1126/science.1099993

25. Baba, M., Nakajo, S., Tu, P. H., Tomita, T., Nakaya, K., Lee, V. M. Y., ... Iwatsubo, T. (1998). Aggregation of $\alpha$-synuclein in Lewy bodies of sporadic Parkinson's disease and dementia with Lewy bodies. American Journal of Pathology, 152(4), 879-884.

26. Wood, J. G., Mirra, S. S., Pollock, N. J., \& Binder, L. I. (1986). Neurofibrillary tangles of Alzheimer disease share antigenic determinants with the axonal microtubule-associated protein tau $(\tau)$. Proceedings of the National Academy of Sciences of the United States of America, 83(11), 4040-4043.

https://doi.org/10.1073/pnas.83.11.4040

27. Scherzinger, E., Lurz, R., Turmaine, M., Mangiarini, L., Hollenbach, B., Hasenbank, R., ... Wanker, E. E. (1997). Huntingtin-encoded polyglutamine expansions form amyloid-like protein aggregates in vitro and in vivo. Cell, 90(3), 549-558. https://doi.org/10.1016/S0092-8674(00)80514-0

28. Benkler, C., O’Neil, A. L., Slepian, S., Qian, F., Weinreb, P. H., \& Rubin, L. L. (2018). Aggregated SOD1 causes selective death of cultured human motor neurons. Scientific Reports, 8(1), 1-14. https://doi.org/10.1038/s41598-018$34759-z$

29. Pickford, F., Masliah, E., Britschgi, M., Lucin, K., Narasimhan, R., Jaeger, P. A., ... Wyss-Coray, T. (2008). The autophagy-related protein beclin 1 shows reduced expression in early Alzheimer disease and regulates amyloid $\beta$ accumulation in mice. Journal of Clinical Investigation, 118(6), 2190-2199. https://doi.org/10.1172/JCI33585

30. Winslow, A. R., \& Rubinsztein, D. C. (2011). The Parkinson disease protein $\alpha-$ synuclein inhibits autophagy. Autophagy, 7(4), 429-431. https://doi.org/10.4161/auto.7.4.14393 
31. Pallanck, L., \& Greenamyre, J. T. (2006). Neurodegenerative disease: Pink, parkin and the brain. Nature, 441(7097), 1058. https://doi.org/10.1038/4411058a

32. Nixon, R. A. (2013). The role of autophagy in neurodegenerative disease. Nature Medicine, 19(8), 983-997. https://doi.org/10.1038/nm.3232

33. Park, J.-S., Koentjoro, B., Veivers, D., Mackay-Sim, A., \& Sue, C. M. (2014). Parkinson's disease-associated human ATP13A2 (PARK9) deficiency causes zinc dyshomeostasis and mitochondrial dysfunction. Human Molecular Genetics, 23(11), 2802-2815. https://doi.org/10.1093/hmg/ddt623

34. Hodges, A., Strand, A. D., Aragaki, A. K., Kuhn, A., Sengstag, T., Hughes, G., ... Luthi-Carter, R. (2006). Regional and cellular gene expression changes in human Huntington's disease brain. Human Molecular Genetics, 15(6), 965-977. https://doi.org/10.1093/hmg/dd1013

35. Metzger, S., Walter, C., Riess, O., Roos, R. A. C., Nielsen, J. E., Craufurd, D., ... Quarrell, O. (2013). The V471A Polymorphism in Autophagy-Related Gene ATG7 Modifies Age at Onset Specifically in Italian Huntington Disease Patients. PLoS ONE, 8(7), 1-9. https://doi.org/10.1371/journal.pone.0068951

36. Di Paolo, G., \& De Camilli, P. (2006). Phosphoinositides in cell regulation and membrane dynamics. Nature, 443(7112), 651-657. https://doi.org/10.1038/nature05185

37. Dall'Armi, C., Devereaux, K. A., \& Di Paolo, G. (2013). The Role of Lipids in the Control of Autophagy. Current Biology, 23(1), R33-R45. https://doi.org/10.1016/j.cub.2012.10.041

38. Gingras, A. C., Kennedy, S. G., O’Leary, M. A., Sonenberg, N., \& Hay, N. (1998). 4E-BP1, a repressor of mRNA translation, is phosphorylated and inactivated by the Akt(PKB) signaling pathway. Genes and Development, 12(4), 502-513. https://doi.org/10.1101/gad.12.4.502 
39. Franke, T. F., Yang, S. Il, Chan, T. O., Datta, K., Kazlauskas, A., Morrison, D. K., ... Tsichlis, P. N. (1995). The protein kinase encoded by the Akt protooncogene is a target of the PDGF-activated phosphatidylinositol 3-kinase. Cell, 81(5), 727-736. https://doi.org/10.1016/0092-8674(95)90534-0

40. Alessi, D. R., James, S. R., Downes, C. P., Holmes, A. B., Gaffney, P. R. J., Reese, C. B., \& Cohen, P. (1997). Characterization of a 3-phosphoinositidedependent protein kinase which phosphorylates and activates protein kinase $\mathrm{B} \alpha$. Current Biology, 7(4), 261-269. https://doi.org/10.1016/s0960-9822(06)00122-9

41. Castro, A. F., Rebhun, J. F., Clark, G. J., \& Quilliam, L. A. (2003). Rheb binds tuberous sclerosis complex 2 (TSC2) and promotes S6 kinase activation in a rapamycin- and farnesylation-dependent manner. Journal of Biological Chemistry, 278(35), 32493-32496. https://doi.org/10.1074/jbc.C300226200

42. Garami, A., Zwartkruis, F. J. T., Nobukuni, T., Joaquin, M., Roccio, M., Stocker, H., ... Thomas, G. (2003). Insulin activation of Rheb, a mediator of mTOR/S6K/4E-BP signaling, is inhibited by TSC1 and 2. Molecular Cell, 11(6), 1457-1466. https://doi.org/10.1016/S1097-2765(03)00220-X

43. Hosokawa, N., Hara, T., Kaizuka, T., Kishi, C., Takamura, A., Miura, Y., ... Mizushima, N. (2009). Nutrient-dependent mTORC1 Association with the ULK1-Atg13-FIP200 Complex Required for Autophagy. Molecular Biology of the Cell, 20(7), 1981-1991. https://doi.org/10.1091/mbc.e08-12-1248

44. Petiot, A., Ogier-denis, E., Blommaart, E. F. C., Meijer, A. J., \& Codogno, P. (2000). Distinct Classes of Phosphatidylinositol 3 -Kinases Are Involved in Signaling Pathways That Control Macroautophagy in HT-29 Cells * possibility that PI3K signaling controls the macroauto-, 275(2), 992-998.

45. Obara, K., \& Ohsumi, Y. (2011). PtdIns 3-Kinase Orchestrates Autophagosome Formation in Yeast, 2011. https://doi.org/10.1155/2011/498768 
46. Obara, K., Sekito, T., \& Ohsumi, Y. (2006). Assortment of Phosphatidylinositol 3-Kinase Complexes - Atg14p Directs Association of Complex I to the Preautophagosomal Structure in Saccharomyces cerevisiae, 17(April), 1527-1539. https://doi.org/10.1091/mbc.E05

47. Zhou, X., Takatoh, J., \& Wang, F. (2011). The Mammalian Class 3 PI3K (PIK3C3) Is Required for Early Embryogenesis and Cell Proliferation. PLoS ONE, 6(1), e16358. https://doi.org/10.1371/journal.pone.0016358

48. Jaber, N., Dou, Z., Chen, J. S., Catanzaro, J., Jiang, Y. P., Ballou, L. M., ... Zong, W. X. (2012). Class III PI3K Vps34 plays an essential role in autophagy and in heart and liver function. Proceedings of the National Academy of Sciences of the United States of America, 109(6), 2003-2008.

https://doi.org/10.1073/pnas.1112848109

49. Obara, K., Noda, T., Niimi, K., \& Ohsumi, Y. (2008). Transport of phosphatidylinositol 3-phosphate into the vacuole via autophagic membranes in Saccharomyces cerevisiae. Genes to Cells, 13(6), 537-547. https://doi.org/10.1111/j.1365-2443.2008.01188.x

50. Simonsen, A. (2004). Alfy, a novel FYVE-domain-containing protein associated with protein granules and autophagic membranes. Journal of Cell Science, 117(18), 4239-4251. https://doi.org/10.1242/jcs.01287

51. Filimonenko, M., Isakson, P., Finley, K. D., Anderson, M., Jeong, H., Melia, T. J., ... Yamamoto, A. (2010). The Selective Macroautophagic Degradation of Aggregated Proteins Requires the PI3P-Binding Protein Alfy. Molecular Cell, 38(2), 265-279. https://doi.org/10.1016/j.molcel.2010.04.007

52. Yang, S. F., Freer, S., \& Benson, A. A. (1967). Transphosphatidylation by phospholipase D. 
53. Fang, Y., Bachmann, R., Flanigan, A., Fang, Y., \& Bachmann, R. (2016).

Phosphatidic Acid-Mediated Mitogenic Activation of mTOR Signaling Published by: American Association for the Advancement of Science Linked references are available on JSTOR for this article : Phosphatidic Acid-Mediated Mitogenic Activation of mTOR Signal, 294(5548), 1942-1945.

54. Shahnazari, S., Yen, W.-L., Birmingham, C. L., Shiu, J., Namolovan, A., Zheng, Y. T., ... Brumell, J. H. (2010). A Diacylglycerol-Dependent Signaling Pathway Contributes to Regulation of Antibacterial Autophagy. Cell Host \& Microbe, 8(2), 137-146. https://doi.org/10.1016/j.chom.2010.07.002

55. Bedia, C., Levade, T., \& Codogno, P. (2011). Regulation of Autophagy by Sphingolipids. Anti-Cancer Agents in Medicinal Chemistry, 11(9), 844-853. https://doi.org/10.2174/187152011797655131

56. Pattingre, S., Bauvy, C., Carpentier, S., Levade, T., Levine, B., \& Codogno, P. (2009). Role of JNK1-dependent Bcl-2 phosphorylation in ceramide-induced macroautophagy. Journal of Biological Chemistry, 284(5), 2719-2728. https://doi.org/10.1074/jbc.M805920200

57. Lavieu, G., Scarlatti, F., Sala, G., Carpentier, S., Levade, T., Ghidoni, R., ... Codogno, P. (2006). Regulation of autophagy by sphingosine kinase 1 and its role in cell survival during nutrient starvation. Journal of Biological Chemistry, 281(13), 8518-8527. https://doi.org/10.1074/jbc.M506182200

58. Berger, J., \& Moller, D. E. (2002). The Mechanisms of Action of PPARs. Annual Review of Medicine, 53(1), 409-435. https://doi.org/10.1146/annurev.med.53.082901.104018

59. Miyata, K. S., McCaw, S. E., Marcus, S. L., Rachubinski, R. A., \& Capone, J. P. (1994). The peroxisome proliferator-activated receptor interacts with the retinoid $\mathrm{X}$ receptor in vivo. Gene, 148(2), 327-330. https://doi.org/10.1016/03781119(94)90707-2 
60. Bull, A. W., Steffensen, K. R., Leers, J., \& Rafter, J. J. (2003). Activation of PPAR $\gamma$ in colon tumor cell lines by oxidized metabolites of linoleic acid, endogenous ligands for PPAR $\gamma$. Carcinogenesis, 24(11), 1717-1722. https://doi.org/10.1093/carcin/bgg131

61. Velkov, T. (2013). Interactions between human liver fatty acid binding protein and peroxisome proliferator activated receptor selective drugs. PPAR Research, 2013, 17-19. https://doi.org/10.1155/2013/938401

62. Nolte, R. T., Wisely, G. B., Westin, S., Cobb, J. E., Lambert, M. H., Kurokawa, R., ... Milburn, M. V. (1998). Ligand binding and co-activator assembly of the peroxisome proliferator- activated receptor- $\gamma$. Nature, 395(6698), 137-143. https://doi.org/10.1038/25931

63. Xu, H. E., Lambert, M. H., Montana, V. G., Plunket, K. D., Moore, L. B., Collins, J. L., ... Willson, T. M. (2001). Structural determinants of ligand binding selectivity between the peroxisome proliferator-activated receptors. Proceedings of the National Academy of Sciences of the United States of America, 98(24), 13919-13924. https://doi.org/10.1073/pnas.241410198

64. Evans, R. M., Barish, G. D., \& Wang, Y.-X. (2004). PPARs and the complex journey to obesity. Nature Medicine, 10(4), 355-361.

https://doi.org/10.1038/nm1025

65. Kim, Y. S., Lee, H.-M., Kim, J. K., Yang, C.-S., Kim, T. S., Jung, M., ... Jo, E.K. (2017). PPAR- $\alpha$ Activation Mediates Innate Host Defense through Induction of TFEB and Lipid Catabolism. The Journal of Immunology, 198(8), 3283-3295. https://doi.org/10.4049/jimmunol.1601920

66. Palomer, X., Capdevila-Busquets, E., Botteri, G., Salvadó, L., Barroso, E., Davidson, M. M., ... Vázquez-Carrera, M. (2014). PPAR $\beta / \delta$ attenuates palmitateinduced endoplasmic reticulum stress and induces autophagic markers in human cardiac cells. International Journal of Cardiology, 174(1), 110-118.

https://doi.org/10.1016/j.ijcard.2014.03.176 
67. Assumpção, J. A. F., Magalhães, K. G., \& Corrêa, J. R. (2017). The role of ppary and autophagy in ros production, lipid droplets biogenesis and its involvement with colorectal cancer cells modulation. Cancer Cell International, 17(1), 1-12. https://doi.org/10.1186/s12935-017-0451-5

68. Zhou, J., Zhang, W., Liang, B., Casimiro, M. C., Whitaker-Menezes, D., Wang, M., ... Wang, C. (2009). PPAR $\gamma$ activation induces autophagy in breast cancer cells. International Journal of Biochemistry and Cell Biology, 41(11), 2334-2342. https://doi.org/10.1016/j.biocel.2009.06.007

69. Wang, C. W. (2015). Lipid droplet dynamics in budding yeast. Cellular and Molecular Life Sciences, 72(14), 2677-2695. https://doi.org/10.1007/s00018-0151903-5

70. Schroeder, B., Schulze, R. J., Weller, S. G., Sletten, A. C., Casey, C. A., \& McNiven, M. A. (2015). The small GTPase Rab7 as a central regulator of hepatocellular lipophagy. Hepatology, 61(6), 1896-1907.

https://doi.org/10.1002/hep.2766

71. Sathyanarayan, A., Mashek, M. T., \& Mashek, D. G. (2017). ATGL Promotes Autophagy/Lipophagy via SIRT1 to Control Hepatic Lipid Droplet Catabolism. Cell Reports, 19(1), 1-9. https://doi.org/10.1016/j.celrep.2017.03.026

72. Settembre, C., De Cegli, R., Mansueto, G., Saha, P. K., Vetrini, F., Visvikis, O., ... Ballabio, A. (2013). TFEB controls cellular lipid metabolism through a starvation-induced autoregulatory loop. Nature Cell Biology, 15(6), 647-658. https://doi.org/10.1038/ncb2718

73. Lettieri Barbato, D., Tatulli, G., Aquilano, K., \& Ciriolo, M. R. (2013). FoxO1 controls lysosomal acid lipase in adipocytes: Implication of lipophagy during nutrient restriction and metformin treatment. Cell Death and Disease, 4(10), e86111. https://doi.org/10.1038/cddis.2013.404 
74. Yokota, S. I., Ando, M., Aoyama, S., Nakamura, K., \& Shibata, S. (2016). Leucine restores murine hepatic triglyceride accumulation induced by a lowprotein diet by suppressing autophagy and excessive endoplasmic reticulum stress. Amino Acids, 48(4), 1013-1021. https://doi.org/10.1007/s00726-0152149-0

75. Irungbam, K., Churin, Y., Matono, T., Weglage, J., Ocker, M., Glebe, D., ... Roeb, E. (2020). Cannabinoid receptor 1 knockout alleviates hepatic steatosis by downregulating perilipin 2. Laboratory Investigation, 100(3), 454-465. https://doi.org/10.1038/s41374-019-0327-5

76. Bajaj, M., Suraamornkul, S., Kashyap, S., Cusi, K., Mandarino, L., \& DeFronzo, R. A. (2004). Sustained reduction in plasma free fatty acid concentration improves insulin action without altering plasma adipocytokine levels in subjects with strong family history of type 2 diabetes. Journal of Clinical Endocrinology and Metabolism, 89(9), 4649-4655. https://doi.org/10.1210/jc.2004-0224

77. Komiya, K., Uchida, T., Ueno, T., Koike, M., Abe, H., Hirose, T., ... Watada, H. (2010). Free fatty acids stimulate autophagy in pancreatic $\beta$-cells via JNK pathway. Biochemical and Biophysical Research Communications, 401(4), 561567. https://doi.org/10.1016/j.bbrc.2010.09.101

78. Suffixidharan, S., Jain, K., \& Basu, A. (2011). Regulation of autophagy by kinases. Cancers, 3(2), 2630-2654. https://doi.org/10.3390/cancers3022630

79. Tan, S. H., Shui, G., Zhou, J., Li, J. J. E., Bay, B. H., Wenk, M. R., \& Shen, H. M. (2012). Induction of autophagy by palmitic acid via protein kinase C-mediated signaling pathway independent of mTOR (mammalian target of rapamycin). Journal of Biological Chemistry, 287(18), 14364-14376. https://doi.org/10.1074/jbc.M111.294157

80. Tu, Q. Q., Zheng, R. Y., Li, J., Hu, L., Chang, Y. X., Li, L., ... Wang, H. Y. (2014). Palmitic acid induces autophagy in hepatocytes via JNK2 activation. Acta Pharmacologica Sinica, 35(4), 504-512. https://doi.org/10.1038/aps.2013.170 
81. Russo, S. B., Baicu, C. F., Van Laer, A., Geng, T., Kasiganesan, H., Zile, M. R., \& Cowart, L. A. (2012). Ceramide synthase 5 mediates lipid-induced autophagy and hypertrophy in cardiomyocytes. Journal of Clinical Investigation, 122(11), 3919-3930. https://doi.org/10.1172/JCI63888

82. Krauss-Etschmann, S., Shadid, R., Campoy, C., Hoster, E., Demmelmair, H., Jiménez, M., ... Koletzko, B. V. (2007). Effects of fish-oil and folate supplementation of pregnant women on maternal and fetal plasma concentrations of docosahexaenoic acid and eicosapentaenoic acid: A European randomized multicenter trial. American Journal of Clinical Nutrition, 85(5), 1392-1400. https://doi.org/10.1093/ajcn/85.5.1392

83. Shin, S., Jing, K., Jeong, S., Kim, N., Song, K. S., Heo, J. Y., ... Lim, K. (2013). The omega-3 polyunsaturated fatty acid DHA induces simultaneous apoptosis and autophagy via mitochondrial ROS-mediated Akt-mTOR signaling in prostate cancer cells expressing mutant p53. BioMed Research International, 2013(c). https://doi.org/10.1155/2013/568671

84. Yao, Q. H., Zhang, X. C., Fu, T., Gu, J. Z., Wang, L., Wang, Y., ... Guo, Y. (2014). $\omega-3$ polyunsaturated fatty acids inhibit the proliferation of the lung adenocarcinoma cell line A549 in vitro. Molecular Medicine Reports, 9(2), 401406. https://doi.org/10.3892/mmr.2013.1829

85. Hsu, H. C., Chen, C. Y., Chiang, C. H., \& Chen, M. F. (2014). Eicosapentaenoic acid attenuated oxidative stress-induced cardiomyoblast apoptosis by activating adaptive autophagy. European Journal of Nutrition, 53(2), 541-547.

https://doi.org/10.1007/s00394-013-0562-2

86. (Lim, K., Hwang, B.-D., Yoon, W.-H., Park, S.-K., Kweon, G.-R., Wu, T., ... Seo. (2011). Docosahexaenoic acid induces autophagy through p53/AMPK/mTOR signaling and promotes apoptosis in human cancer cells harboring wild-type p53. Autophagy, 7(11), 1348-1358. Retrieved from http://dx.doi.org/10.4161/auto.7.11.16658 
87. Tasdemir, E., Maiuri, M. C., Galluzzi, L., Vitale, I., Djavaheri-Mergny, M., D'Amelio, M., ... Kroemer, G. (2008). Regulation of autophagy by cytoplasmic p53. Nature Cell Biology, 10(6), 676-687. https://doi.org/10.1038/ncb1730

88. Vilimanovich, U., Bosnjak, M., Bogdanovic, A., Markovic, I., Isakovic, A., Kravic-Stevovic, T., ... Bumbasirevic, V. (2015). Statin-mediated inhibition of cholesterol synthesis induces cytoprotective autophagy in human leukemic cells. European Journal of Pharmacology, 765, 415-428. https://doi.org/10.1016/j.ejphar.2015.09.004

89. Gatticchi, L., Bellezza, I., Del Sordo, R., Peirce, M. J., Sidoni, A., Roberti, R., \& Minelli, A. (2015). The Tm7sf2 gene deficiency protects mice against endotoxininduced acute kidney injury. PLoS ONE, 10(11), 1-13.

https://doi.org/10.1371/journal.pone.0141885

90. Dehay B, Martinez-Vicente M, Caldwell GA, Caldwell KA, Yue Z, Cookson MR, Klein C, Vila M, Bezard E. Lysosomal impairment in Parkinson's disease. Mov Disord. 2013 Jun;28(6):725-732. PMID: 23580333

91. Hara T, Nakamura K, Matsui M, Yamamoto A, Nakahara Y, Suzuki-Migishima R, Yokoyama M, Mishima K, Saito I, Okano H, Mizushima N. Suppression of basal autophagy in neural cells causes neurodegenerative disease in mice. Nature. Nature Publishing Group; 2006 Jun;441(7095):885-889.

92. Tanik SA, Schultheiss CE, Volpicelli-Daley LA, Brunden KR, Lee VMY. Lewy body-like $\alpha$-synuclein aggregates resist degradation and impair macroautophagy. $\mathrm{J}$ Biol Chem. 2013 May 24;288(21):15194-15210. PMCID: PMC3663539

93. Jakobi AJ, Huber ST, Mortensen SA, Schultz SW, Palara A, Kuhm T, Shrestha BK, Lamark T, Hagen WJH, Wilmanns M, Johansen T, Brech A, Sachse C. Structural basis of p62/SQSTM1 helical filaments and their role in cellular cargo uptake. Nat Commun. 2020 Jan 23;11(1):440. PMCID: PMC6978347 
94. Kim D-H, Sarbassov DD, Ali SM, King JE, Latek RR, Erdjument-Bromage H, Tempst P, Sabatini DM. mTOR interacts with raptor to form a nutrient-sensitive complex that signals to the cell growth machinery. Cell. 2002 Jul 26;110(2):163175. PMID: 12150925

95. Chmurzyńska A. The multigene family of fatty acid-binding proteins (FABPs): function, structure and polymorphism. J Appl Genet. 2006;47(1):39-48. PMID: 16424607

96. Smathers RL, Petersen DR. The human fatty acid-binding protein family: evolutionary divergences and functions. Hum Genomics. 2011 Mar;5(3):170-191. PMCID: PMC3500171

97. Storch J, Thumser AE. Tissue-specific functions in the fatty acid-binding protein family. J Biol Chem. 2010 Oct 22;285(43):32679-32683. PMCID: PMC2963392

98. Ewing RM, Chu P, Elisma F, Li H, Taylor P, Climie S, McBroom-Cerajewski L, Robinson MD, O’Connor L, Li M, Taylor R, Dharsee M, Ho Y, Heilbut A, Moore L, Zhang S, Ornatsky O, Bukhman YV, Ethier M, Sheng Y, Vasilescu J, AbuFarha M, Lambert J-P, Duewel HS, Stewart II, Kuehl B, Hogue K, Colwill K, Gladwish K, Muskat B, Kinach R, Adams S-L, Moran MF, Morin GB, Topaloglou T, Figeys D. Large-scale mapping of human protein-protein interactions by mass spectrometry. Mol Syst Biol. 2007;3:89. PMCID: PMC 1847948

99. Lv Q, Wang G, Zhang Y, Han X, Li H, Le W, Zhang M, Ma C, Wang P, Ding Q. FABP5 regulates the proliferation of clear cell renal cell carcinoma cells via the PI3K/AKT signaling pathway. Int J Oncol. 2019 Apr;54(4):1221-1232. PMCID: PMC6411348

100. Zhou Q, Li J, Wang H, Yin Y, Zhou J. Identification of nigral dopaminergic neuron-enriched genes in adult rats. Neurobiol Aging. 2011 Feb;32(2):313-326. PMID: 19303663 
101. Biedler JL, Roffler-Tarlov S, Schachner M, Freedman LS. Multiple neurotransmitter synthesis by human neuroblastoma cell lines and clones. Cancer Res. 1978 Nov;38(11 Pt 1):3751-3757. PMID: 29704

102. Brown NA, Kemp JA, Seabrook GR. Block of human voltage-sensitive Na+ currents in differentiated SH-SY5Y cells by lifarizine. Br J Pharmacol. 1994 Oct;113(2):600-606. PMCID: PMC1510106

103. Sonnier H, Kolomytkin O, Marino A. Action potentials from human neuroblastoma cells in magnetic fields. Neurosci Lett. 2003 Feb 13;337(3):163166. PMID: 12536049

104. Krishna A, Biryukov M, Trefois C, Antony PMA, Hussong R, Lin J, Heinäniemi M, Glusman G, Köglsberger S, Boyd O, van den Berg BHJ, Linke D, Huang D, Wang K, Hood L, Tholey A, Schneider R, Galas DJ, Balling R, May P. Systems genomics evaluation of the SH-SY5Y neuroblastoma cell line as a model for Parkinson's disease. BMC Genomics. 2014;15:1154. PMCID: PMC4367834

105. Korecka JA, van Kesteren RE, Blaas E, Spitzer SO, Kamstra JH, Smit AB, Swaab DF, Verhaagen J, Bossers K. Phenotypic characterization of retinoic acid differentiated SH-SY5Y cells by transcriptional profiling. PLoS ONE. 2013;8(5):e63862. PMCID: PMC3665836

106. Shipley MM, Mangold CA, Szpara ML. Differentiation of the SH-SY5Y Human Neuroblastoma Cell Line. J Vis Exp. 2016 Feb 17;(108):53193. PMCID: PMC4828168

107. Mu YM, Yanase T, Nishi Y, Tanaka A, Saito M, Jin CH, Mukasa C, Okabe T, Nomura M, Goto K, Nawata H. Saturated FFAs, palmitic acid and stearic acid, induce apoptosis in human granulosa cells. Endocrinology. 2001 Aug;142(8):3590-3597. PMID: 11459807

108. Zeng L, Wu G-Z, Goh KJ, Lee YM, Ng CC, You AB, Wang J, Jia D, Hao A, Yu Q, Li B. Saturated fatty acids modulate cell response to DNA damage: implication for their role in tumorigenesis. PLoS ONE. 2008 Jun 4;3(6):e2329. PMCID: PMC2402972 
109. Pu J, Peng G, Li L, Na H, Liu Y, Liu P. Palmitic acid acutely stimulates glucose uptake via activation of Akt and ERK1/2 in skeletal muscle cells. J Lipid Res. 2011 Jul;52(7):1319-1327. PMCID: PMC3122917

110. Agholme L, Lindström T, Kågedal K, Marcusson J, Hallbeck M. An in vitro model for neuroscience: differentiation of SH-SY5Y cells into cells with morphological and biochemical characteristics of mature neurons. J Alzheimers Dis. 2010;20(4):1069-1082. PMID: 20413890

111. Påhlman S, Ruusala AI, Abrahamsson L, Mattsson ME, Esscher T. Retinoic acidinduced differentiation of cultured human neuroblastoma cells: a comparison with phorbolester-induced differentiation. Cell Differ. 1984 Jun;14(2):135-144. PMID: 6467378

112. Cheung Y-T, Lau WK-W, Yu M-S, Lai CS-W, Yeung S-C, So K-F, Chang RC-C. Effects of all-trans-retinoic acid on human SH-SY5Y neuroblastoma as in vitro model in neurotoxicity research. Neurotoxicology. 2009 Jan;30(1):127-135. PMID: 19056420

113. Klionsky DJ, Abdel-Aziz AK, Abdelfatah S, Abdellatif M, Abdoli A, Abel S, Abeliovich H, ... Tong C-K. Guidelines for the use and interpretation of assays for monitoring autophagy (4th edition)1. Autophagy. 2021 Jan;17(1):1-382. PMCID: PMC7996087

114. N'Diaye E-N, Kajihara KK, Hsieh I, Morisaki H, Debnath J, Brown EJ. PLIC proteins or ubiquilins regulate autophagy-dependent cell survival during nutrient starvation. EMBO Rep. 2009 Feb;10(2):173-179. PMCID: PMC2637314

115. Thijssen MA, Mensink RP. Small differences in the effects of stearic acid, oleic acid, and linoleic acid on the serum lipoprotein profile of humans. Am J Clin Nutr. 2005 Sep;82(3):510-516. PMID: 16155261

116. Zarini S, Murphy RC. Biosynthesis of 5-oxo-6,8,11,14-eicosatetraenoic acid from 5-hydroperoxyeicosatetraenoic acid in the murine macrophage. J Biol Chem. 2003 Mar 28;278(13):11190-11196. PMID: 12547823 
117. Rodríguez JP, Guijas C, Astudillo AM, Rubio JM, Balboa MA, Balsinde J. Sequestration of 9-Hydroxystearic Acid in FAHFA (Fatty Acid Esters of Hydroxy Fatty Acids) as a Protective Mechanism for Colon Carcinoma Cells to Avoid Apoptotic Cell Death. Cancers (Basel). 2019 Apr 12;11(4). PMCID: PMC6521239

118. Kuda O, Brezinova M, Silhavy J, Landa V, Zidek V, Dodia C, Kreuchwig F, Vrbacky M, Balas L, Durand T, Hübner N, Fisher AB, Kopecky J, Pravenec M. Nrf2-Mediated Antioxidant Defense and Peroxiredoxin 6 Are Linked to Biosynthesis of Palmitic Acid Ester of 9-Hydroxystearic Acid. Diabetes. 2018;67(6):1190-1199. PMID: 29549163

119. Pizato N, Luzete BC, Kiffer LFMV, Corrêa LH, de Oliveira Santos I, Assumpção JAF, Ito MK, Magalhães KG. Omega-3 docosahexaenoic acid induces pyroptosis cell death in triple-negative breast cancer cells. Sci Rep. 2018 31;8(1):1952. PMCID: PMC5792438

120. Guilbert M, Ferland C, Bossé M, Flamand N, Lavigne S, Laviolette M. 5-Oxo6,8,11,14-eicosatetraenoic acid induces important eosinophil transmigration through basement membrane components: comparison of normal and asthmatic eosinophils. Am J Respir Cell Mol Biol. 1999 Jul;21(1):97-104. PMID: 10385597

121. Doherty J, Baehrecke EH. Life, death and autophagy. Nat Cell Biol. 2018 Oct;20(10):1110-1117. PMID: 30224761

122. Saxton RA, Sabatini DM. mTOR Signaling in Growth, Metabolism, and Disease. Cell. 2017 Mar 9;168(6):960-976. PMCID: PMC5394987

123. Mariño G, Niso-Santano M, Baehrecke EH, Kroemer G. Self-consumption: the interplay of autophagy and apoptosis. Nat Rev Mol Cell Biol. 2014 Feb;15(2):8194. PMCID: PMC3970201

124. Mulcahy Levy JM, Thorburn A. Autophagy in cancer: moving from understanding mechanism to improving therapy responses in patients. Cell Death Differ. 2020 Mar;27(3):843-857. PMCID: PMC7206017 
125. Funk CD. Prostaglandins and leukotrienes: advances in eicosanoid biology. Science. 2001 Nov 30;294(5548):1871-1875. PMID: 11729303

126. Powell WS, Rokach J. The eosinophil chemoattractant 5-oxo-ETE and the OXE receptor. Prog Lipid Res. 2013 Oct;52(4):651-665. PMCID: PMC5710732

127. Stamatiou P, Hamid Q, Taha R, Yu W, Issekutz TB, Rokach J, Khanapure SP, Powell WS. 5-oxo-ETE induces pulmonary eosinophilia in an integrin-dependent manner in Brown Norway rats. J Clin Invest. 1998 Dec 15;102(12):2165-2172. PMCID: PMC509171

128. Erlemann K-R, Rokach J, Powell WS. Oxidative stress stimulates the synthesis of the eosinophil chemoattractant 5-oxo-6,8,11,14-eicosatetraenoic acid by inflammatory cells. J Biol Chem. 2004 Sep 24;279(39):40376-40384. PMID: 15234979

129. Morrison BE, Marcondes MCG, Nomura DK, Sanchez-Alavez M, SanchezGonzalez A, Saar I, Kim K-S, Bartfai T, Maher P, Sugama S, Conti B. Cutting edge: IL-13R $\alpha 1$ expression in dopaminergic neurons contributes to their oxidative stress-mediated loss following chronic peripheral treatment with lipopolysaccharide. J Immunol. 2012 Dec 15;189(12):5498-5502. PMCID: PMC3545403

130. Spittau B, Zhou X, Ming M, Krieglstein K. IL6 protects MN9D cells and midbrain dopaminergic neurons from MPP+-induced neurodegeneration. Neuromolecular Med. 2012 Dec;14(4):317-327. PMID: 22772723

131. Hosoi T, Koguchi Y, Sugikawa E, Chikada A, Ogawa K, Tsuda N, Suto N, Tsunoda S, Taniguchi T, Ohnuki T. Identification of a novel human eicosanoid receptor coupled to G(i/o). J Biol Chem. 2002 Aug 30;277(35):31459-31465. PMID: 12065583 
132. Patel P, Cossette C, Anumolu JR, Gravel S, Lesimple A, Mamer OA, Rokach J, Powell WS. Structural requirements for activation of the 5-oxo-6E,8Z, 11Z,14Zeicosatetraenoic acid (5-oxo-ETE) receptor: identification of a mead acid metabolite with potent agonist activity. J Pharmacol Exp Ther. 2008 May;325(2):698-707. PMID: 18292294

133. Habib NA, Wood CB, Apostolov K, Barker W, Hershman MJ, Aslam M, Heinemann D, Fermor B, Williamson RC, Jenkins WE. Stearic acid and carcinogenesis. Br J Cancer. 1987 Oct;56(4):455-458. PMCID: PMC2001814

134. Senyilmaz-Tiebe D, Pfaff DH, Virtue S, Schwarz KV, Fleming T, Altamura S, Muckenthaler MU, Okun JG, Vidal-Puig A, Nawroth P, Teleman AA. Dietary stearic acid regulates mitochondria in vivo in humans. Nat Commun. 2018 07;9(1):3129. PMCID: PMC6081440

135. Jenkins TC, Abughazaleh AA, Freeman S, Thies EJ. The production of 10hydroxystearic and 10-ketostearic acids is an alternative route of oleic acid transformation by the ruminal microbiota in cattle. J Nutr. 2006 Apr;136(4):926931. PMID: 16549452

136. Calonghi N, Cappadone C, Pagnotta E, Farruggia G, Buontempo F, Boga C, Brusa GL, Santucci MA, Masotti L. 9-Hydroxystearic acid upregulates p21(WAF1) in HT29 cancer cells. Biochem Biophys Res Commun. 2004 Jan 30;314(1):138-142. PMID: 14715257

137. Arakaki AK, Mezencev R, Bowen NJ, Huang Y, McDonald JF, Skolnick J. Identification of metabolites with anticancer properties by computational metabolomics. Mol Cancer. 2008 Jun 17;7:57. PMCID: PMC2453147

138. Schmidt A, Endo N, Rutledge SJ, Vogel R, Shinar D, Rodan GA. Identification of a new member of the steroid hormone receptor superfamily that is activated by a peroxisome proliferator and fatty acids. Mol Endocrinol. 1992 Oct;6(10):16341641. PMID: 1333051 
139. Armstrong EH, Goswami D, Griffin PR, Noy N, Ortlund EA. Structural basis for ligand regulation of the fatty acid-binding protein 5, peroxisome proliferatoractivated receptor $\beta / \delta$ (FABP5-PPAR $\beta / \delta$ ) signaling pathway. J Biol Chem. 2014 May 23;289(21):14941-14954. PMCID: PMC4031543 
APPENDIX A 


\section{Central Nervous System and Peripheral Cell Labeling by Vascular Endothelial Cadherin-Driven Lineage Tracing in Adult Mice}

Citation: Soto-Avellaneda, A., \& Morrison, B. (2020). Central nervous system and peripheral cell labeling by vascular endothelial cadherin-driven lineage tracing in adult mice. Neural Regeneration Research, 15(10), 1856-1866. https://doi.org/10.4103/16735374.280317

\section{$\underline{\text { Abstract }}$}

Understanding the contribution of endothelial cells to the progenitor pools of adult tissues has the potential to inform therapies for human disease. To address whether endothelial cells transdifferentiate into non-vascular cell types, we performed cell lineage tracing analysis using transgenic mice engineered to express a fluorescent marker following activation by tamoxifen in vascular endothelial cadherin promoter-expressing cells (VEcad-CreER ${ }^{T 2} ;$ B6 Cg-Gt(ROSA)26Sort $\left.{ }^{m 9(C A G-t d T o m a t o) H z e}\right)$. Activation of target-cell labeling following 1.5 months of ad libitum feeding with tamoxifen-laden chow in 4-5 month-old mice resulted in the tracing of central nervous system and peripheral cells that include: cerebellar granule neurons, ependymal cells, skeletal myocytes, pancreatic beta cells, pancreatic acinar cells, tubular cells in the renal cortex, duodenal crypt cells, ileal crypt cells, and hair follicle stem cells. As Nestin expression has been reported in a subset of endothelial cells, $\mathrm{Nes}$-CreER ${ }^{T 2}$ mice were also utilized in these conditions. The tracing of cells in adult $\mathrm{Nes}-\mathrm{CreER} \mathrm{R}^{T 2}$ mice revealed the labeling of canonical progeny cell types such as hippocampal and olfactory granule neurons as well as ependymal cells. Interestingly, Nestin tracing also labeled skeletal myocytes, ileal crypt cells, and sparsely marked cerebellar granule neurons. Our findings provide support for endothelial cells as 
active contributors to adult tissue progenitor pools. This information could be of particular significance for the intravenous delivery of therapeutics to downstream endothelialderived cellular targets. The animal experiments were approved by the Boise State University Institute Animal Care and Use Committee (approval No. 006-AC15-018) on October 31, 2018.

Introduction

Identifying cell populations in adult mammals that undergo regeneration and determining the source of progenitors can inform therapeutic strategies for a wide range of human diseases. For such strategies to succeed, consideration must be given to the contributions of non-canonical mechanisms for tissue regeneration. Supporting this notion is accumulating evidence indicating the existence of naturally occurring transdifferentiation mechanisms for adult cell replenishment (Michalopoulos et al., 2005; Tang et al., 2012; Tarlow et al., 2014; Merrell and Stanger, 2016). Endothelial cells are key players in human disease (Donato et al., 2015) while also representing an understudied potential pool of adult progenitor cells. These cells are particularly promising candidates due to the possibility of using intravenous genetic manipulation to target difficult to reach cell populations, such as those of the central nervous system, in conjunction with vectors currently employed in clinical trials (e.g., adeno-associated virus). Endothelial transdifferentiation would also offer an explanation for reported extravascular effects following intravenous delivery of viral vectors in humans and laboratory model systems. The proximity to all tissue types and a well-described association with adult progenitor cells (e.g., the perivascular stem cell niche) (Oh and Nör, 2015; Tamplin et al., 2015) provide additional rationale for further examination of endothelial cells in this context. 
A growing body of evidence supports adult endothelial cell plasticity. For example, primary mouse neural progenitor cells have been observed to spontaneously differentiate into endothelial cells in culture (Wurmser et al., 2004). In addition, there is evidence that populations of adult neuronal progenitor cells migrate from the meninges, a region possessing a high concentration of endothelial cells (Bifari et al., 2017). Another area of attracting increased interest is the study of the endothelial-mesenchymal transition, which is a natural transdifferentiation phenomenon undertaken by endothelial cells and is an element of normal cardiac development (Bernanke and Markwald, 1982; Mjaatvedt et al., 1987; Eisenberg and Markwald, 1995; Camenisch et al., 2002; de Lange et al., 2004) that may also underlie several forms of vascular disease (Chen et al., 2015; Moonen et al., 2015; Li et al., 2018). Lastly, there is evidence that in adult rodents, specialized endothelial cells can dedifferentiate to become smooth muscle cells and chondrogenic cells following vascular injury (Tang et al., 2012). These multipotent endothelial cells have been reported to readily differentiate into diverse cell types, including neurons in vitro (Tang et al., 2012). Taken together, these findings blur the line between endothelial cells and other cell types, suggesting that endothelial cells could be attractive targets for cell lineage tracing analysis in an adult mammalian system.

The development of innovative cell lineage tracing approaches has accelerated research efforts in the field of regenerative medicine. Traditional cell lineage tracing methods that rely upon the labeling of replicated DNA (e.g., BrdU, [3H]-thymidine) would be expected to underreport transdifferentiation events due to the absence of cell division in this process. To address the limitations of traditional approaches, cell lineage tracing strategies that utilize a permanent fluorescent reporter activated by temporally- 
controlled genetic alteration have become prevalent (Kretzschmar and Watt, 2012).

Therefore, to carry out a comprehensive cell lineage tracing study of endothelial transdifferentiation, we employed such a strategy that utilized an adult transgenic mouse model for specific temporal labeling of endothelial cells. Mice were sacrificed at varied intervals following the labeling period and 23 tissue types were assessed for the presence (or absence) of a fluorescent marker to identify cellular progeny.

\section{Materials and Methods}

$\underline{\text { Animals }}$

All mouse procedures and husbandry were approved by the Boise State University Institute Animal Care and Use Committee (approval No. 006-AC15-018) on October 31, 2018. Mice were maintained in the Boise State University rodent vivarium at $25^{\circ} \mathrm{C}$ with 12 hour light/dark cycles and provided food ad libitum. VEcad-CreERT2 transgenic mice were kindly gifted by University of California, Los Angeles, USA (Monvoisin et al., 2006). NesCreERT2 transgenic mice (stock\# 016261) (Battiste et al., 2007) and B6 CgGt(ROSA)26Sor ${ }^{\text {tm9(CAG-tdTomato)Hze }}$ reporter mice (stock\# 007909) (Madisen et al., 2010) were purchased from Jackson laboratory (Bar Harbor, ME, USA). Cell lineage tracing experiments were initiated by replacing standard rodent pellets with chow containing $400 \mathrm{mg}$ tamoxifen citrate/kg chow (Cat\# TD.130860; Envigo, Huntingdon, Cambridgeshire, UK) as described previously (Albright et al., 2016; Rahman et al., 2017). After 1.5 months of tamoxifen treatment, the chow was replaced with standard rodent pellets. Both female and male 4-5month-old mice were used in this study and no sex-linked variation in lineage tracing was observed. Genotyping for transgenic mouse lines was performed as described previously 
(Monvoisin et al., 2006; Battiste et al., 2007; Madisen et al., 2010). At least two mice per cohort were examined for reporter expression.

\section{Tissue Preparation and Cryosectioning}

Study mice were anesthetized with isoflurane followed by transcardial perfusion with heparin $(20 \mathrm{U} / \mathrm{mL})$ in neutral phosphate buffer $(\mathrm{PB})$ and then $4 \%$ paraformaldehyde (PFA) in PB. Tissue was harvested and placed in ice-cold 4\%PFA/PB and stored for 24 hours at $4{ }^{\circ} \mathrm{C}$. Samples were then transferred to a $30 \%$ sucrose/PB solution for storage at $4^{\circ} \mathrm{C}$ for 72 hours. Tissue was then dried and frozen rapidly in optimal cutting temperature (OCT) media on a block of dry ice. Samples were then wrapped in aluminum foil, placed in freezer bags and stored at $-80^{\circ} \mathrm{C}$ until sectioning. Tissues were equilibrated to $-20^{\circ} \mathrm{C}$ and then sectioned using a cryostat (model CM1950; Leica, Buffalo Grove, IL, USA) at a thickness of $15 \mu \mathrm{m}$ directly onto Superfrost Plus Gold microscope slides (Thermo Fisher, Waltham, MA, USA). Slides were dried in a dark drawer overnight. The next day, slides were processed for labeling or placed in a slide holder, sealed, and stored at $-80^{\circ} \mathrm{C}$.

\section{Hoechst Dye Labeling}

Slides were removed from $-80^{\circ} \mathrm{C}$ storage and allowed to equilibrate to room temperature in a dark drawer. Slides were then washed with PB and labeled with a 1:20,000 dilution of Hoechst 33342 dye (Cat\# H3570; Thermo Fisher) for 10 minutes at room temperature. One PB wash was performed and sections were then allowed to dry in a dark drawer for 30 minutes. Coverslips were then mounted using Everbrite mounting media (Cat\# 23003; Biotium, Fremont, CA, USA). Slides were allowed to dry in a dark drawer for 24 hours and then stored in a slide box at $4^{\circ} \mathrm{C}$ until viewed. 


\section{Immunohistochemistry}

Section-containing slides were removed from $-80^{\circ} \mathrm{C}$ storage and dried for 1 hour in the dark at room temperature. Antigen retrieval was performed as previously described (Hussaini et al., 2013). Slides were washed with PB and then placed in a container with citrate solution (Cat\# 14746S; Cell Signaling, Danvers, MA, USA) diluted in $\mathrm{dH}_{2} \mathrm{O}$ that was preheated in a microwave ( 5 minutes at high power). The solution with slides was then heated in a microwave for an additional 7 minutes at high power. The heated container was then covered and placed in an ice bath for 1 hour. Slides were then washed three times with a Tris solution (50 mM Tris $\mathrm{HCl}, 150 \mathrm{mM} \mathrm{NaCl}, 0.05 \%$ Triton X100-not pH adjusted). A pHadjusted Tris solution was used (TBST: $50 \mathrm{mM}$ Tris $\mathrm{HCl}, 150 \mathrm{mM} \mathrm{NaCl}, 0.05 \%$ Triton $\mathrm{X} 100-\mathrm{pH} 7.2$ ) as a final wash. The slides were then dried for 30 minutes and primary antibodies were then diluted in TBST supplemented with donkey serum to 4\%. The primary antibodies used in this study are as follows: anti-mCherry (Cat\# MBS448092; 1:100; Mybiosource, San Diego, CA, USA), anti-NeuN (Cat\# 24307S; 1:100; Cell Signaling), antiinsulin (Cat\# 3014S; 1:800; Cell Signaling), anti-glucagon (Cat\# sc-514592, 1:100; Santa Cruz Biotechnology, Dallas, TX, USA), and anti-K15 (Cat\# sc-47697; 1:100; Santa Cruz Biotechnology). Antibody solutions were incubated in a humidifying slide chamber at room temperature overnight. The next day, slides were washed with TBST and incubated with the appropriate secondary antibodies diluted in TBST with donkey serum to $4 \%$ for 2 hours at room temperature. The secondary antibodies used in this study are as follows: donkey antigoat Alexa 594 (Cat\# A32758; 1:500; Thermo Fisher), donkey anti-mouse Alexa 488 (Cat\# A-21202; 1:500; Thermo Fisher) and donkey anti-rabbit Alexa 488 (Cat\# A-21206; 1:500;

Thermo Fisher). Slides were washed with TBST and then incubated with a 1:20,000 dilution 
of Hoechst 33342 dye (Cat\# H3570; Thermo Fisher) at room temperature for 10 minutes. A final wash in PB was performed and slides were then dried for $1 \mathrm{~h}$ in a dark drawer. Once dry, the slides were mounted with coverslips using Everbrite mounting media (Cat\# 23003; Biotium). The coverslips were allowed to dry for 24 hours in a dark drawer before imaging or storage at $4^{\circ} \mathrm{C}$.

Imaging and assessment of tissue sections

Conventional fluorescence microscopy images were obtained using an EVOS M7000 Imaging System (Thermo Fisher). Hoechst images were viewed using a DAPI filter cube and tdTomato was assessed using an RFP filter cube in the EVOS system. Semi-quantitative assessment of tdTomato+ve cells was performed by comparing three or more comparable levels of a tissue section obtained from at least two mice per cohort. Cells were counted at $100 \times$ fields and a relative number of “+” signs assigned. When comparing across different cohorts within a tissue group, the number of + signs correlate to relative increases or decreases in tdTomato +ve cell number. As such, this method is not stereological but provides a strong correlation with the relative abundance of cells expressing tdTomato. Confocal microscopy images were acquired using the following systems: Zeiss Laser Scanning Confocal Microscope Meta 510 system (Zeiss, White Plains, NY, USA), Zeiss 880 Airy Scan system and an Olympus FV3000 Laser Scanning Confocal Microscope system (Olympus, Center Valley, PA, USA). A supplemental image database containing representative conventional fluorescence microscopy images (EVOS M7000 system) from all examined tissues can be found at the Open Science Framework (https://osf.io/x9yu8). 


\section{Results}

$\underline{\text { VE-cadherin cell lineage tracing labels a diversity of cell types in adult mice }}$

Endothelial cell labeling was performed using a double transgenic mouse system that allows for VE-cadherin promoter-specific expression of a CRE-recombinase ( VEcadCreERT2) (Monvoisin et al., 2006), kindly provided by Dr. Iruela-Arispe (UCLA)) that is activated at designated times by the addition of tamoxifen (TAM) to rodent chow (Figure A1). Following activation by TAM, CreERT2 excises a loxP-flanked DNA cassette containing a stop codon within a transgenic CAG-promoter-driven tdTomato reporter (Gt(ROSA)26Sortm9(CAG-tdTomato)Hze) (Madisen et al., 2010), resulting in permanent and constitutive expression of tdTomato. VE-cadherin-directed CreERT2 expression was selected due to the high degree of endothelial cell specificity reported in adult mice (Heimark et al., 1990; Monvoisin et al., 2006). We also observed prototypical endothelial cell tracing in these mice (Additional Figure 1). For this study, we sought to extend the labeling period to 1.5 months to enhance the tagging of transient endothelial cell populations not covered by previous studies (Figure A1B). In addition, we lengthened the post-labeling phase $(1.5,3$, and 6 months) for increased capture of slow transdifferentiation events. A summary of the results for the examined cell types and tissues is found in (Table A1). In addition, a repository of images containing transgenic mouse lines, tissues, and experimental time points has been provided in Additional file 1. 


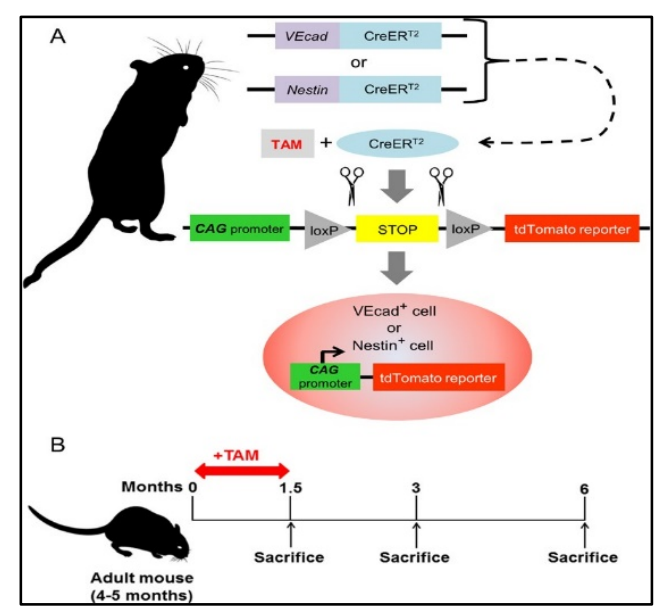

Figure A1 Transgenic mouse cell lineage tracing strategy. (A). Adult mice (4-5 months of age) possessing a transgenic cassette containing either vascular endothelial cadherin (VEcad)- or Nestin-promoter-driven.CreER ${ }^{\mathrm{T} 2}$ recombinase are fed tamoxifen (TAM) citrate-laden chow to acti- vate loxP site-directed excision of a second transgene (Ai9 line) possessing a translation stop codon sequence. Removal of this stop codon results in CAG promoter-driven reporter expression of tdTomato. Note that post-TAM tdTomato expression is solely controlled by the CAG promoter and Gt(RO- SA)26Sor chromosomal locus (tdTomato reporter locus). (B) Permanent activation of tdTomato expression in VEcad or Nestin-expressing cells was achieved by feeding adult mice tamoxifen for 1.5 months. Mice were sacri- ficed at the indicated times, fixed and histological assessment was performed. 
Table A1 Cell lineage tracing results for vascular endothelial cadherin and Nestin

Cell types were scored semi-quantitatively for the relative abundance of tdTomato positive cells $(+)$ at the indicated times following initiation of tamoxifen treatment in (A) VEcadCreER ${ }^{T 2} /$ Rosa-flox-STOP-tdTomato or (B) Nes-CreER T2/Rosa-flox-STOP-tdTomato transgenic mice. Cell types without $(-)$ tdTomato labeling are also shown. Notes: a myocytes very weakly labeled with most signal surrounding the muscle fiber; ${ }^{b_{\text {abundant }} \text { weakly }}$ positive cells; ${ }^{c_{\text {possible }}}$ tdTomato ${ }^{+}$tumor noted; $\mathrm{d}_{\text {weakly positive }}$

\begin{tabular}{|c|c|c|c|c|c|c|c|}
\hline Cell type & $\begin{array}{c}1.5 \text { mon } \\
\text { post- } \\
\text { TAM }\end{array}$ & $\begin{array}{c}3 \text { mon } \\
\text { post- } \\
\text { TAM } \\
\end{array}$ & $\begin{array}{l}6 \text { mon } \\
\text { post- } \\
\text { TAM } \\
\end{array}$ & Cell type & $\begin{array}{c}1.5 \text { mon } \\
\text { post- } \\
\text { TAM }\end{array}$ & $\begin{array}{l}3 \text { mon } \\
\text { post- } \\
\text { TAM } \\
\end{array}$ & $\begin{array}{c}6 \text { mon } \\
\text { post- } \\
\text { TAM }\end{array}$ \\
\hline \multicolumn{4}{|c|}{$\begin{array}{l}\text { A. Vascular endothelial cadherin cell lineage } \\
\text { tracing }\end{array}$} & \multicolumn{4}{|c|}{ B. Nestin cell lineage tracing } \\
\hline $\begin{array}{c}\text { Olfactory } \\
\text { bulb granule } \\
\text { neurons }\end{array}$ & - & - & - & $\begin{array}{c}\text { Olfactory } \\
\text { bulb granule } \\
\text { neurons }\end{array}$ & - & +++++ & $\begin{array}{c}+++++ \\
+\end{array}$ \\
\hline $\begin{array}{c}\text { Olfactory } \\
\text { bulb } \\
\text { juxtaglomer } \\
\text { ular } \\
\text { projections }\end{array}$ & ++ & ++++ & ++ & $\begin{array}{c}\text { Olfactory } \\
\text { bulb } \\
\text { juxtaglomer } \\
\text { ular } \\
\text { projections }\end{array}$ & + & ++++ & ++ \\
\hline $\begin{array}{c}\text { Midbrain } \\
\text { neurons }\end{array}$ & - & - & - & $\begin{array}{l}\text { Midbrain } \\
\text { neurons }\end{array}$ & - & - & - \\
\hline $\begin{array}{l}\text { Midbrain } \\
\text { ventricular } \\
\text { cells } \\
\end{array}$ & - & - & - & $\begin{array}{c}\text { Midbrain } \\
\text { ventricular } \\
\text { cells } \\
\end{array}$ & - & +++++ & ++ \\
\hline $\begin{array}{l}\text { Cortical } \\
\text { neurons }\end{array}$ & - & - & - & $\begin{array}{l}\text { Cortical } \\
\text { neurons }\end{array}$ & - & - & - \\
\hline $\begin{array}{l}\text { Hippocamp } \\
\text { al neurons }\end{array}$ & - & - & - & $\begin{array}{l}\text { Hippocampa } \\
1 \text { neurons }\end{array}$ & - & + & ++ \\
\hline $\begin{array}{l}\text { Cerebellar } \\
\text { granule } \\
\text { neurons }\end{array}$ & ++++ & $\begin{array}{c}+++++ \\
+\end{array}$ & - & $\begin{array}{l}\text { Cerebellar } \\
\text { granule } \\
\text { neurons }\end{array}$ & - & ++ & + \\
\hline $\begin{array}{l}\text { Choroid } \\
\text { plexus }\end{array}$ & +++ & +++++ & +++ & $\begin{array}{l}\text { Choroid } \\
\text { plexus }\end{array}$ & ++ & - & +++ \\
\hline $\begin{array}{r}\text { Striatal } \\
\text { neurons }\end{array}$ & - & - & - & $\begin{array}{c}\text { Striatal } \\
\text { neurons }\end{array}$ & - & - & - \\
\hline $\begin{array}{c}\text { Subventricu } \\
\text { lar zone } \\
\text { layer I }\end{array}$ & - & ++ & - & $\begin{array}{c}\text { Subventricul } \\
\text { ar zone } \\
\text { layer I }\end{array}$ & - & $\begin{array}{c}++++++ \\
++\end{array}$ & ++++++ \\
\hline $\begin{array}{l}\text { Subventricu } \\
\text { lar zone } \\
\text { layers } 2-4\end{array}$ & - & - & - & $\begin{array}{c}\text { Subventricul } \\
\text { ar zone } \\
\text { layers } 2-4\end{array}$ & - & - & - \\
\hline $\begin{array}{l}\text { Pancreatic } \\
\text { islet cells }\end{array}$ & $\begin{array}{c}++++++ \\
+\end{array}$ & ++++ & - & $\begin{array}{l}\text { Pancreatic } \\
\text { islet cells }\end{array}$ & - & - & - \\
\hline
\end{tabular}




\begin{tabular}{|c|c|c|c|c|c|c|c|}
\hline Cell type & $\begin{array}{c}1.5 \text { mon } \\
\text { post- } \\
\text { TAM } \\
\end{array}$ & $\begin{array}{c}3 \text { mon } \\
\text { post- } \\
\text { TAM } \\
\end{array}$ & $\begin{array}{l}6 \text { mon } \\
\text { post- } \\
\text { TAM } \\
\end{array}$ & Cell type & $\begin{array}{c}1.5 \text { mon } \\
\text { post- } \\
\text { TAM } \\
\end{array}$ & $\begin{array}{c}3 \text { mon } \\
\text { post- } \\
\text { TAM } \\
\end{array}$ & $\begin{array}{l}6 \text { mon } \\
\text { post- } \\
\text { TAM } \\
\end{array}$ \\
\hline \multicolumn{4}{|c|}{$\begin{array}{l}\text { A. Vascular endothelial cadherin cell lineage } \\
\text { tracing }\end{array}$} & \multicolumn{4}{|c|}{ B. Nestin cell lineage tracing } \\
\hline $\begin{array}{l}\text { Pancreatic } \\
\text { acinar cells }\end{array}$ & $\begin{array}{c}++++++ \\
+\end{array}$ & ++++ & - & $\begin{array}{l}\text { Pancreatic } \\
\text { acinar cells }\end{array}$ & - & - & + \\
\hline $\begin{array}{c}\text { Skeletal } \\
\text { myocytes } \\
\text { (hamstring) }\end{array}$ & ++ & ++++++ & $++^{\mathrm{a}}$ & $\begin{array}{c}\text { Skeletal } \\
\text { myocytes } \\
\text { (hamstring) }\end{array}$ & - & +++ & + \\
\hline $\begin{array}{c}\text { Skeletal } \\
\text { myocytes } \\
\text { (underlying } \\
\text { dorsal skin) }\end{array}$ & - & - & ++++ & $\begin{array}{c}\text { Skeletal } \\
\text { myocytes } \\
\text { (underlying } \\
\text { dorsal skin) }\end{array}$ & - & + & +++ \\
\hline $\begin{array}{c}\text { Cardiac } \\
\text { myocytes }\end{array}$ & - & - & - & $\begin{array}{c}\text { Cardiac } \\
\text { myocytes }\end{array}$ & - & - & - \\
\hline Hepatocytes & - & - & - & Hepatocytes & - & - & - \\
\hline $\begin{array}{c}\text { Renal cells } \\
\text { (cortical } \\
\text { layer) }\end{array}$ & +++++ & +++ & - & Renal cells & - & - & - \\
\hline $\begin{array}{l}\text { Hair follicle } \\
\text { cells }\end{array}$ & $\overline{+++++}$ & ++ & - & $\begin{array}{l}\text { Hair follicle } \\
\text { cells }\end{array}$ & - & - & - \\
\hline $\begin{array}{l}\text { Splenic } \\
\text { follicles }\end{array}$ & - & - & - & $\begin{array}{l}\text { Splenic } \\
\text { follicles }\end{array}$ & - & - & - \\
\hline $\begin{array}{l}\text { Gastric pit } \\
\text { cells }\end{array}$ & - & - & - & $\begin{array}{l}\text { Gastric pit } \\
\text { cells }\end{array}$ & - & - & - \\
\hline $\begin{array}{l}\text { Duodenal } \\
\text { crypt cells }\end{array}$ & +++ & - & - & $\begin{array}{l}\text { Duodenal } \\
\text { crypt cells }\end{array}$ & - & - & - \\
\hline $\begin{array}{l}\text { Ileal crypt } \\
\text { cells }\end{array}$ & $\begin{array}{c}++++++ \\
++++\end{array}$ & - & - & $\begin{array}{l}\text { Ileal crypt } \\
\text { cells }\end{array}$ & $\begin{array}{c}++++++ \\
++^{\mathrm{b}}\end{array}$ & $-^{c}$ & $++^{\mathrm{d}}$ \\
\hline $\begin{array}{l}\text { Colonic } \\
\text { crypt cells }\end{array}$ & & & & $\begin{array}{l}\text { Colonic } \\
\text { crypt cells }\end{array}$ & - & - & - \\
\hline
\end{tabular}

\section{Central Nervous System}

Examination of the central nervous system (CNS) following TAM treatment revealed tdTomato-positive cells in several cell types not previously reported to share embryonic origins with endothelial cells. Positive cellular projections were observed in the olfactory bulb adjacent to but outside of glomeruli, suggesting that these cells are not olfactory neurons (Figure A2). These projections were noted in a juxtaglomerular region within all levels of 
olfactory bulb sections and at all post-TAM treatment time points. No associated somas were observed, suggesting that these projections originated outside of the olfactory bulb.

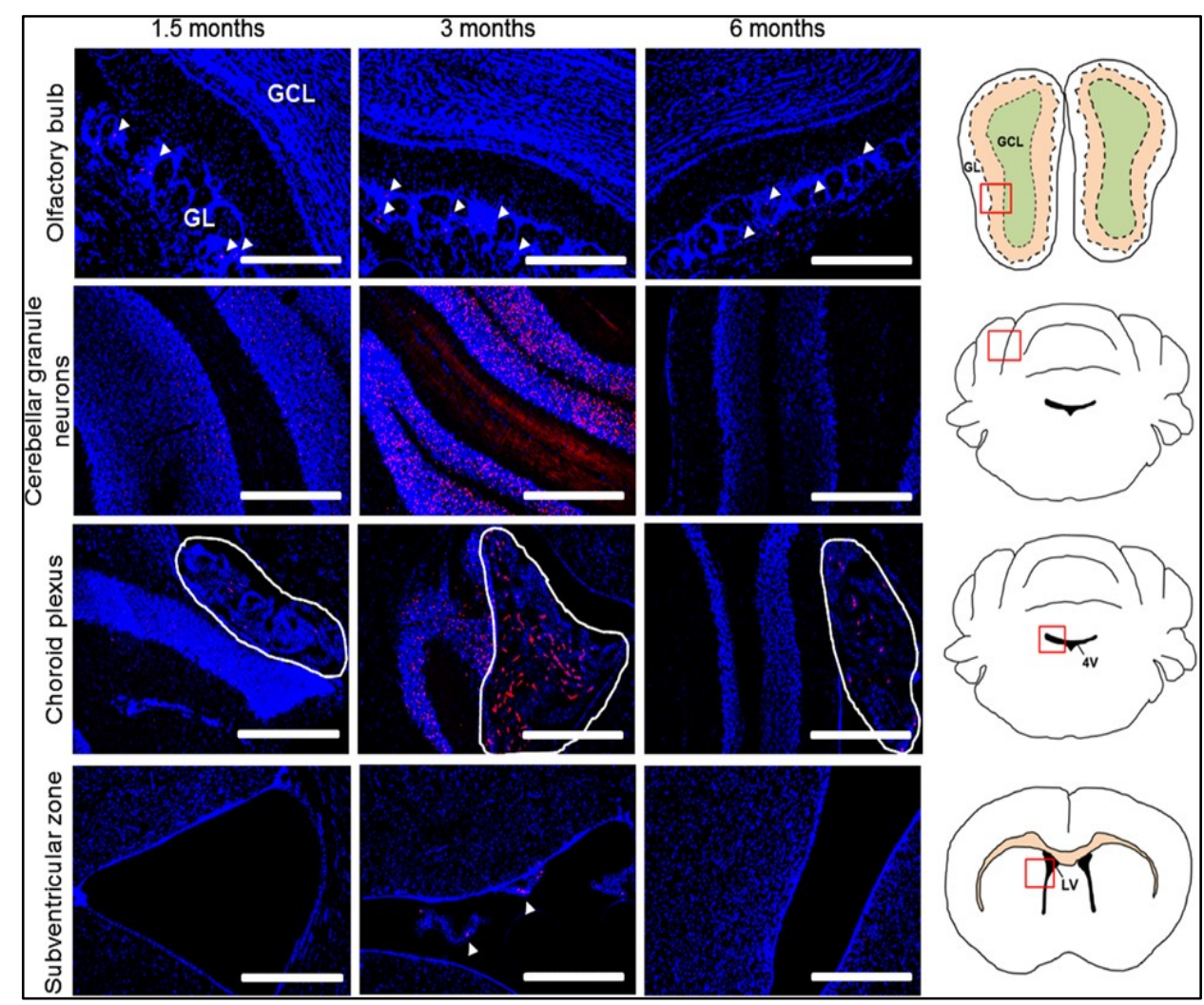

Figure A2 Vascular endothelial-cadherin cell fate mapping in the central nervous system. Adult mice possessing VEcad-CreERT2/Rosa-flox-STOP-tdTomato were fed tamoxifen-containing chow for $\mathbf{1 . 5}$ months and mice sacrificed at the indicated times following initiation of treatment. Representative channel merged images from fixed and Hoechst labeled tissues. Nuclei are labeled by Hoechst (blue) and cell lineage tracing is indicated by tdTomato (red). The areas shown in micrographs are indicated by highlighted regions (red boxes) in diagrams to the right where the granule cell layer (GCL), glomerular layer (GL), lateral ventricle (LV) and fourth ventricle (4V) are shown. Arrowheads highlight labeled cells and cellular appendages (white scale bars: $400 \mu \mathrm{m}$ ).

Interestingly, numerous tdTomato + cells were detected in the granule layer of the cerebellum (Figure A2). Positive cellular projections within the molecular layer were detected and confocal microscopic examination (Figure A3A, AS2, and AS4) confirmed that the abundant positive cells in this region were cerebellar granule neurons (CGNs). A clear 
gradation of positive CGN expression was observed, with a peak at 3 months post-TAM treatment and with complete absence observed at 6 months. The choroid plexus lining the ventricle ventral to the cerebellum was tdTomato + at all time points, with peak frequency of tdTomato expression occurring 3 months following TAM treatment. The subventricular zone (SVZ), a region possessing adult olfactory bulb neuronal progenitors, exhibited positive labeling only in the ependymal layer (SVZ layer I) at 3 months of age (Figure A2).

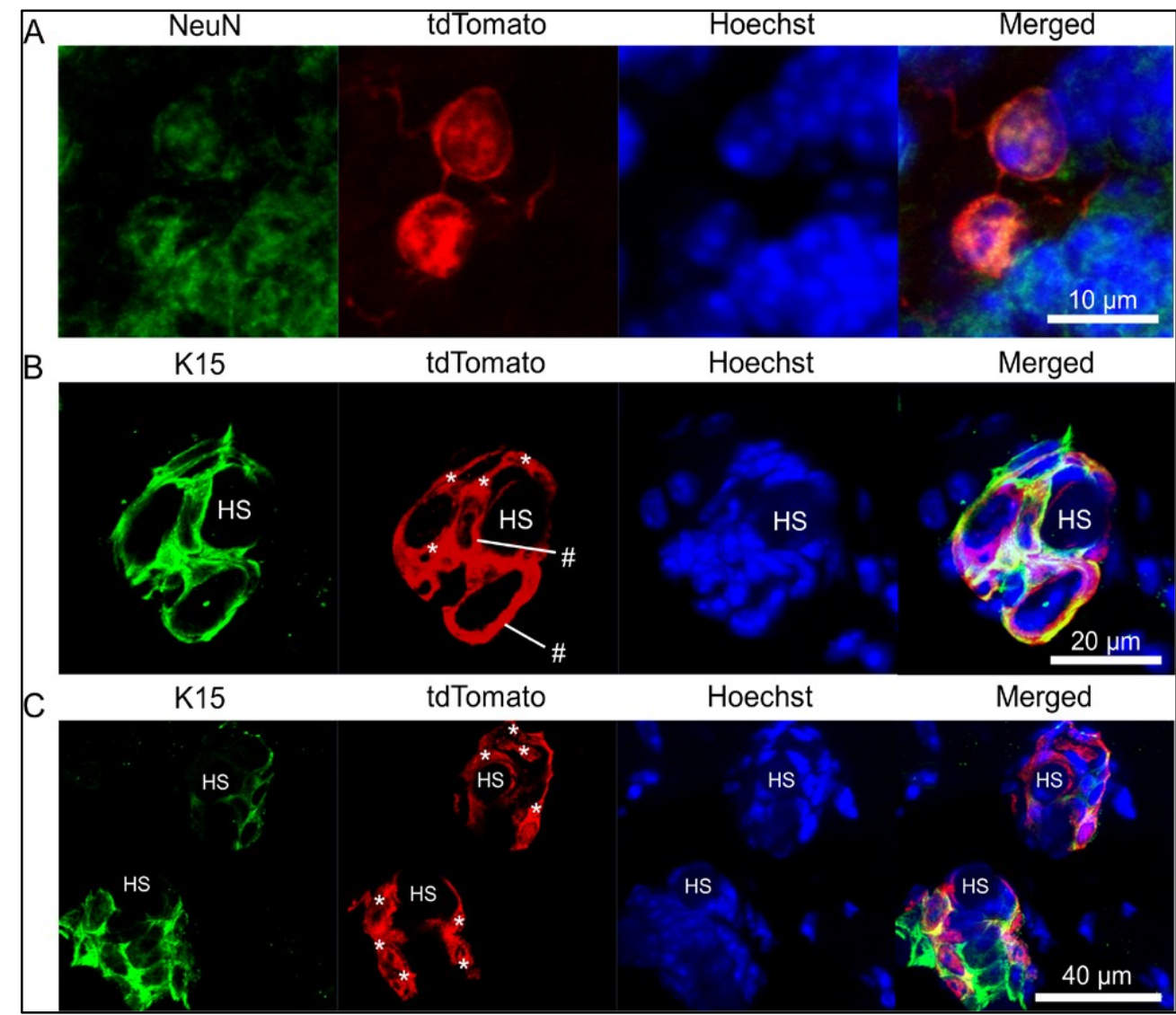

Figure A3 Cerebellar granule neurons and hair follicle stem cells express vascular endothelial-cadherin fate mapping reporter. Representative confocal microscopy images from adult $\mathrm{VEcad}$-CreER $\mathrm{R}^{\mathrm{T}} / \mathrm{Rosa}$-flox-STOP-tdTomato transgenic mouse cerebellum

(A) and skin $(B, C)$ at three months following the start of tamoxifen treatment. Immunohis- tochemical labeling for (A) NeuN (green) or (B, C) K15 (green) with tdTomato (red) and nucleus (Hoechst; blue) are shown at two magnifications to demonstrate the rare occurrence of K15-tdTomato colocalization. Images captured using a Zeiss

Laser Scanning Confocal Microscope Meta 510 sys- tem. Orthogonal projections provided in the supplementary data indicate cells exhibiting (\#) red/green colocalization. Asterisks (*) signify cells with- out co-localization, hair shafts (HS) are also shown. 


\section{$\underline{\text { Hair follicle stem cells }}$}

Inspection of integumentary tissue obtained from the dorsal pelt of adult transgenic mice revealed tdTomato expression within hair root tissue (Figure A4). The most robust labeling was observed 1.5 months after TAM treatment, with labeling decreasing at 3 months and reaching undetectable levels at 6 months. Confocal microscopic assessment (Figure $3 \mathrm{~A}-\mathrm{C}$ and AS2B-D) revealed that a small portion of tdTomato-expressing cells co-label with the hair root stem cell marker $\mathrm{K} 15$. It is interesting to note the numerous instances in which tdTomato-labeled cells were observed in close proximity to K15-expressing cells without exhibiting co-localization (Figure AS2B-D). 


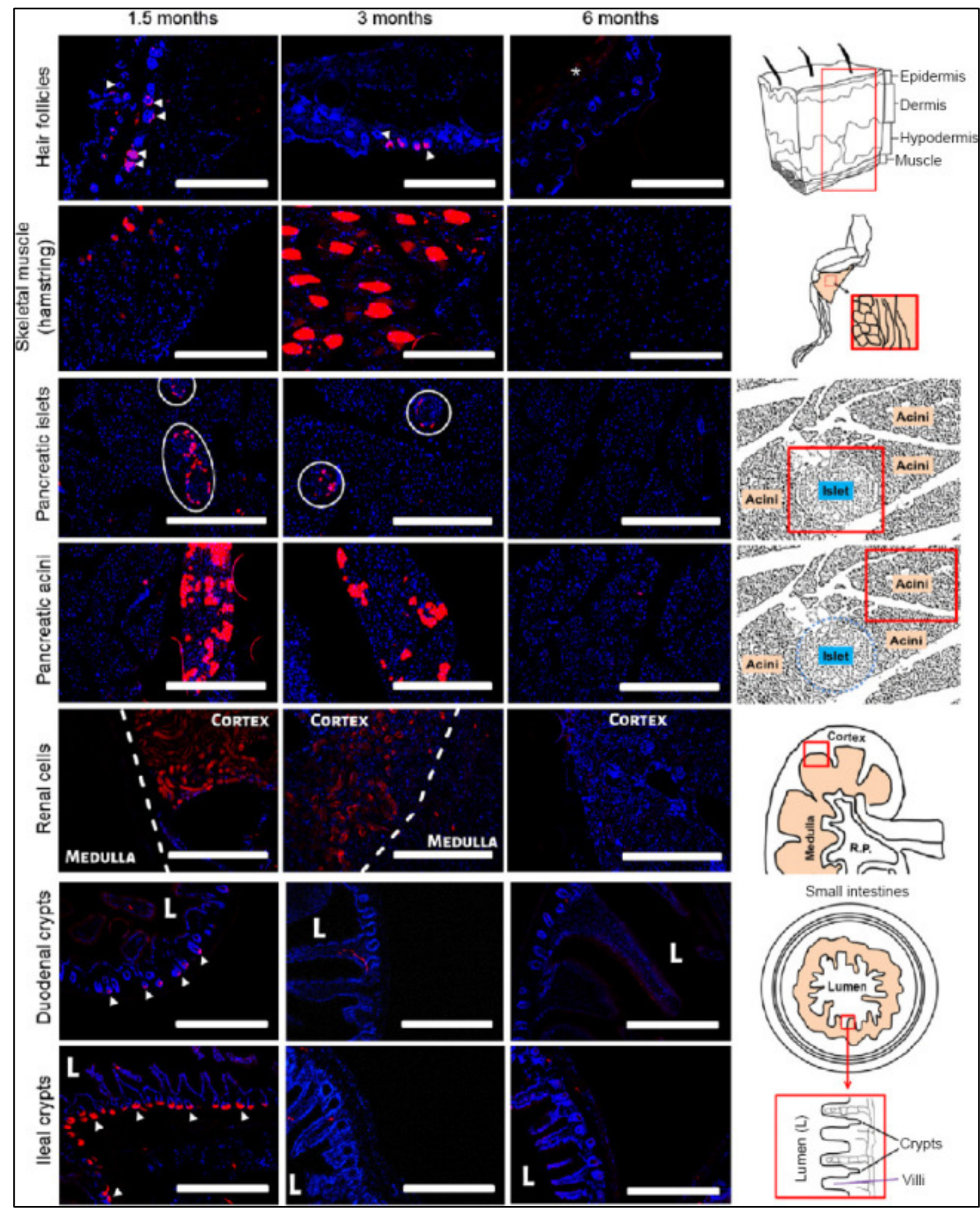

Figure A4 Vascular endothelial-cadherin cell fate mapping in the periphery. 4-5-

Month-old VEcad-CreERT2/Rosa-flox-STOP-tdTomato mice were provided tamoxifen chow for 1.5 months and sacrificed at the times shown. Images were obtained from fixed and Hoechst stained tissues. Cell lineage tracing is evidenced by tdTomato (red) and nuclei are indicated by Hoechst (blue) labeling. Arrowheads highlight labeled cells (white scale bar $=400 \mu \mathrm{m}$ ). Red boxes displayed in diagrams indicate region shown in the associated micrographs. Asterisk (*) indicates tdTomato+ muscle fibers underlying skin; Intestinal lumen (L) and tdTomato+ crypt cells (arrowheads) are labeled. Renal tissue diagram indicates the location of the renal pelvis (R.P.). 


\section{$\underline{\text { Skeletal muscle }}$}

The hamstrings of adult mice were also inspected for tdTomato labeling (Figures A4 and AS3). Surprisingly, extensive labeling was observed at 1.5 months, with maximum labeling observed 3 months after TAM treatment. After 6 months, faint fluorescence was still evident surrounding muscle fibers, indicating labeling within the endomysium region with possible satellite cell involvement. No strongly labeled muscle fibers were observed for that time point. Labeling was concentrated in localized regions of muscle whenever robust tdTomato expression was observed (1.5 and 3 months post-TAM), leaving most areas of muscle with no labeled fibers. In addition, muscle residing beneath the subcutaneous layer of dorsal skin was found to be weakly tdTomato + at 6 months post-TAM with no detectable fluorescence at 1.5 or 3 months (Figure A4).

\section{$\underline{\text { Pancreas }}$}

Another unexpected finding was the observed labeling of pancreatic islets (Figure A4). There was a well-defined gradation of labeling, with all observed islets possessing tdTomato-positive cells 1.5 months post-TAM and with $83 \%$ of islets containing one or more positive cells 3 months post-TAM. No tdTomato+ cells were observed 6 months post-TAM. Confocal microscopic analysis (Figures A5A and AS5A) revealed that some of the positive cells were beta cells ( $3 \%$ insulin+). It is worthwhile to note the extremely close proximity of the tdTomato-positive cells with insulin-positive cells in an interwoven configuration (Figures A5A, B and AS5A, B). Co-labeling with glucagon (Figure A5C and Additional Figure AS5C) was not observed, indicating that none of the tdTomato-expressing cells at these time points were alpha cells. 


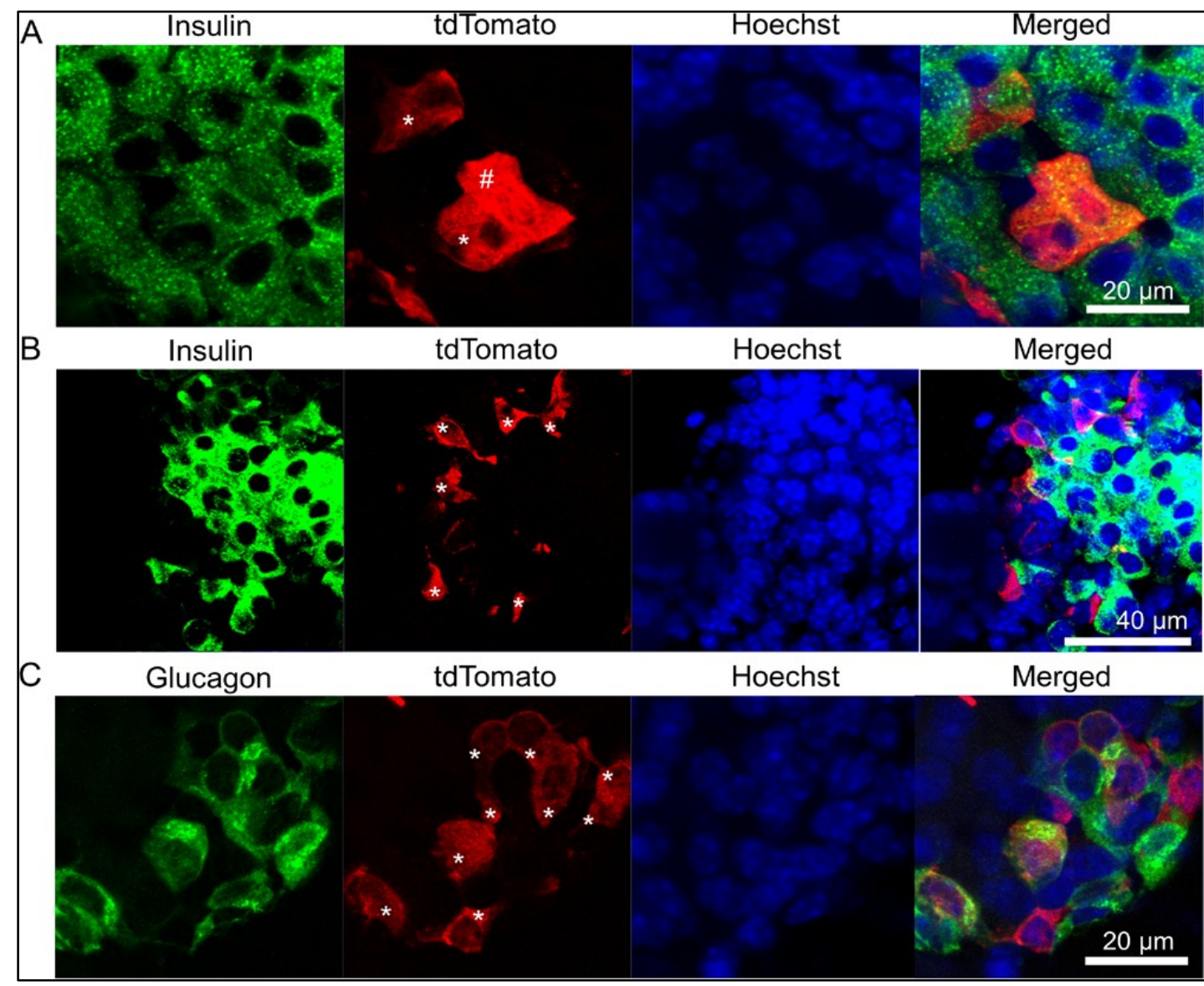

Figure A5 Vascular endothelial-cadherin fate mapping labels pancreatic $\beta$ cells.Immunohistochemistry was performed for pancreatic insulin $(A, B)$ and tdTomato reporter expression at three months post-tamoxifen treatment in adult VEcad$\mathrm{CreER}^{T 2} /$ Rosa-flox-STOP-tdTomato transgenic mice. Representative images were obtained by confocal microscopy for insulin (A, B; green), glucagon (C; green), tdTomato (red) and the nu- cleus (Hoechst; blue). Two magnifications of separate fields are shown to demonstrate the rare instances of Insulin-tdTomato colocalization. A Zeiss Laser Scanning Confocal Microscope Meta 510 system was used to capture and analyze the images shown. Confocal orthogonal projections presented in the supplemental data section were utilized to confirm cells with (\#) or without (*) green/red co-localization

TdTomato labeling was also detected in the acini of the exocrine pancreas (Figures A4 and AS6). The most robust labeling was observed at 1.5 months and decreasing thereafter. Only a small minority of pancreatic acini exhibited positive labeling. Similar to the pattern witnessed in skeletal muscle, positive cells were most often grouped in close 
proximity to each other. Likewise, only a very faint fluorescent signal in acini was detected 6 months post-TAM.

\section{$\underline{\text { Renal Cells }}$}

Kidney sections were assessed and found to exhibit strong tdTomato expression 1.5 months after TAM administration (Figure A4). Labeling was restricted to the cortical layer and markedly absent from the medulla. This suggests that tdTomato labeling was likely confined to proximal and distal convoluted tubules. Expression intensity diminished significantly 3 months post-TAM and was only very weakly detectable in the cortex at 6 months. It should be noted that residual urea would have been flushed from the tissue sections by numerous washes associated with sample preparation before imaging. Therefore, it is not expected that filtrate or urine (which might exhibit tdTomato fluorescence) contributed to the observed signal.

\section{$\underline{\text { Small Intestines }}$}

Examination of the intestinal tract revealed positive tdTomato labeling for crypt cells (crypt of Lieberkühn) found in both the duodenum and ileum (Figure A4). These labeled cells were concentrated within the most basal portion of the intestinal gland. The most intense labeling was observed 1.5 months post-TAM and labeling was completely absent (or very indistinct) 3 and 6 months following TAM treatment. No fluorescence was observed for colonic cells at any time point.

Nestin Cell Lineage Tracing Labels for Canonical and Non-Canonical Targets in Adult Mice

The intermediate filament Nestin is known to exhibit a promiscuous expression pattern. However, it has been reported that some endothelial cells (as well as specialized 
endothelial progenitor cells) express Nestin in adult mice (Mokrý et al., 2008; Suzuki et al., 2010; Ding and Morrison, 2013; Itkin et al., 2016; Dusart et al., 2018). Therefore, Nestin cell lineage tracing (Nes-CreERT2), as employed here (Figure A1), could provide additional information with respect to endothelial cell progeny. We found both canonical Nestin cell progeny as well as novel cell labeling events show some correlation with VE-cadherin cell lineage tracing. A detailed summary of Nestin cell lineage tracing results can be found in Table Aland additional image sets are provided in Additional file 1.

\section{$\underline{\text { Central Nervous System }}$}

Analysis of adult mouse brain (Figure A6) showed tdTomato labeling of neurons in the granule cell layer of the olfactory bulb at 3 months, which was maintained at a similar level 6 months post-TAM. This is consistent with previous reports of Nestin-positive progenitors migrating from the SVZ to establish adult-born olfactory bulb neurons (Altman, 1969; Menezes et al., 1995; Zerlin et al., 1995). Labeled olfactory neurons were not present at 1.5 months, which indicates that the tdTomato-expressing cells at 3 months were indeed derived outside of the olfactory bulb. Interestingly, we observed juxtaglomerular projections in the olfactory bulb at all time points, as was witnessed in VE-cadherin lineage tracing experiments described above. No associated cell somas were noted, which indicates that these projections originated outside of the olfactory bulb. Similar to adult olfactory bulb granule neurons, populations of hippocampal cells are known to undergo adult neurogenesis from Nestin+ progenitors (Palmer et al., 1995). Supporting this notion, we find tdTomatopositive cells in the hippocampus beginning 3 months following TAM administration and increasing at 6 months. Interestingly, we find only a relatively small number of labeled cells 
in this region, despite numerous reports in the literature of abundant neuronal regeneration in the hippocampus of adult mice (Altman and Das, 1965; Bayer et al., 1982).

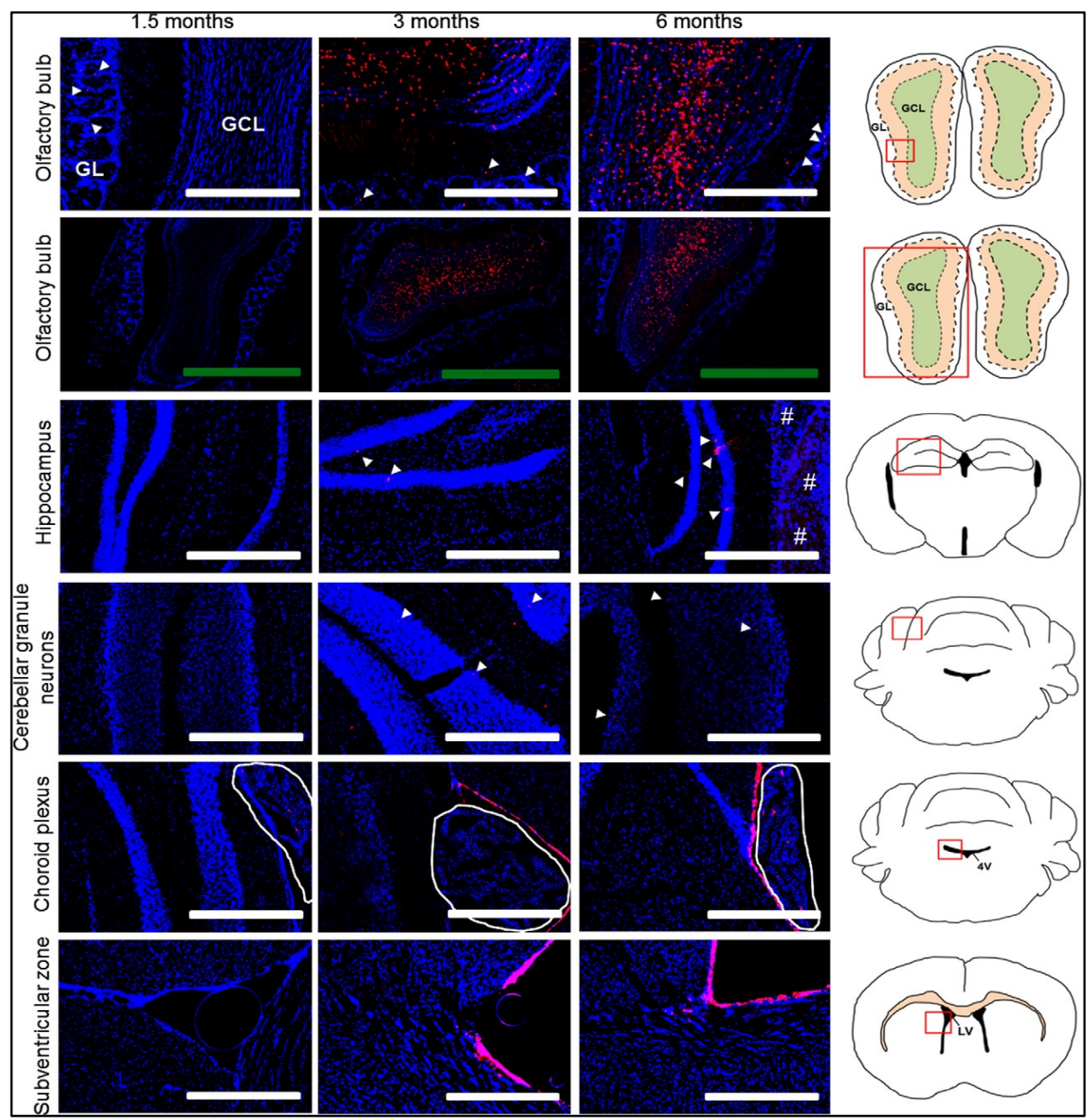

Figure A6 Nestin cell fate mapping in the central nervous system. Transgenic Nes$\mathrm{CreER}^{T 2} /$ Rosa-flox-STOP-tdTomato mice at 4-5 months of age were fed tamoxifen-lad- en chow for 1.5 months and then sacrificed at thetimes shown post treatment initiation. Channel merged images were then acquired from fixed and Hoechst stained tissues. Nuclei are labeled by Hoechst (blue) and cell lineage tracing is indicated by tdTomato (red). Images shown were obtained from the regions indicated by the red box in the associated diagram to the right where the granule cell layer (GCL), glomerular layer (GL), lateral ventricle (LV) and fourth ventricle (4V) are shown. Arrow- heads highlight labeled cells and cellular append- ages; \# indicates autofluorescence from wrinkled tissue region (white scale bars: $400 \mu \mathrm{m}$; green scale bars: $1000 \mu \mathrm{m}$ ). 
Our study also detected tdTomato-labeled cerebellar granule neurons at 3 and 6 months following treatment with TAM (Figure A6). No tdTomato-positive cells were noted in the 1.5-month cohort. Both the 3- and 6-month groups exhibited far fewer positive cerebellar granule neurons compared to our VE-cadherin study. Assessment of the nearby choroid plexus showed sparse labeling at 1.5 and 6 months. Surprisingly, no labeled cells were observed 3 months after TAM treatment for the cohort of mice we examined. This correlates inversely with VE-cadherin labeling of the same structure at 3 months. Another interesting finding was positive labeling of ependymal cells lining the ventricular surfaces extending throughout the brain, which was absent at 1.5 months but robustly labeled 3 and 6 months post-TAM. The expression pattern also shows a clear disconnect between the choroid plexus and the ubiquitous ependymal layer indicating differential regulation for their production.

$\underline{\text { Skeletal Muscle }}$

Investigation of skeletal muscle obtained from hamstring found tdTomato-labeled muscle fibers 3 months after TAM treatment while being absent in the 1.5-month group (Figure A7). However, the 6-month cohort showed positive labeling for very few muscle fibers. It is evident that the majority of positive cells at 3 months have an elevated signal originating from the endomysium area in addition to signals originating from myocytes. Comparable to VE-cadherin tracing, Nestin-traced myocytes were found in close proximity to each other. We also observed robust Nestin tracing to muscle underlying the subcutaneous fat layer of dorsal skin at 3 and 6 months post-TAM but not at 1.5 months (Figure A7). 


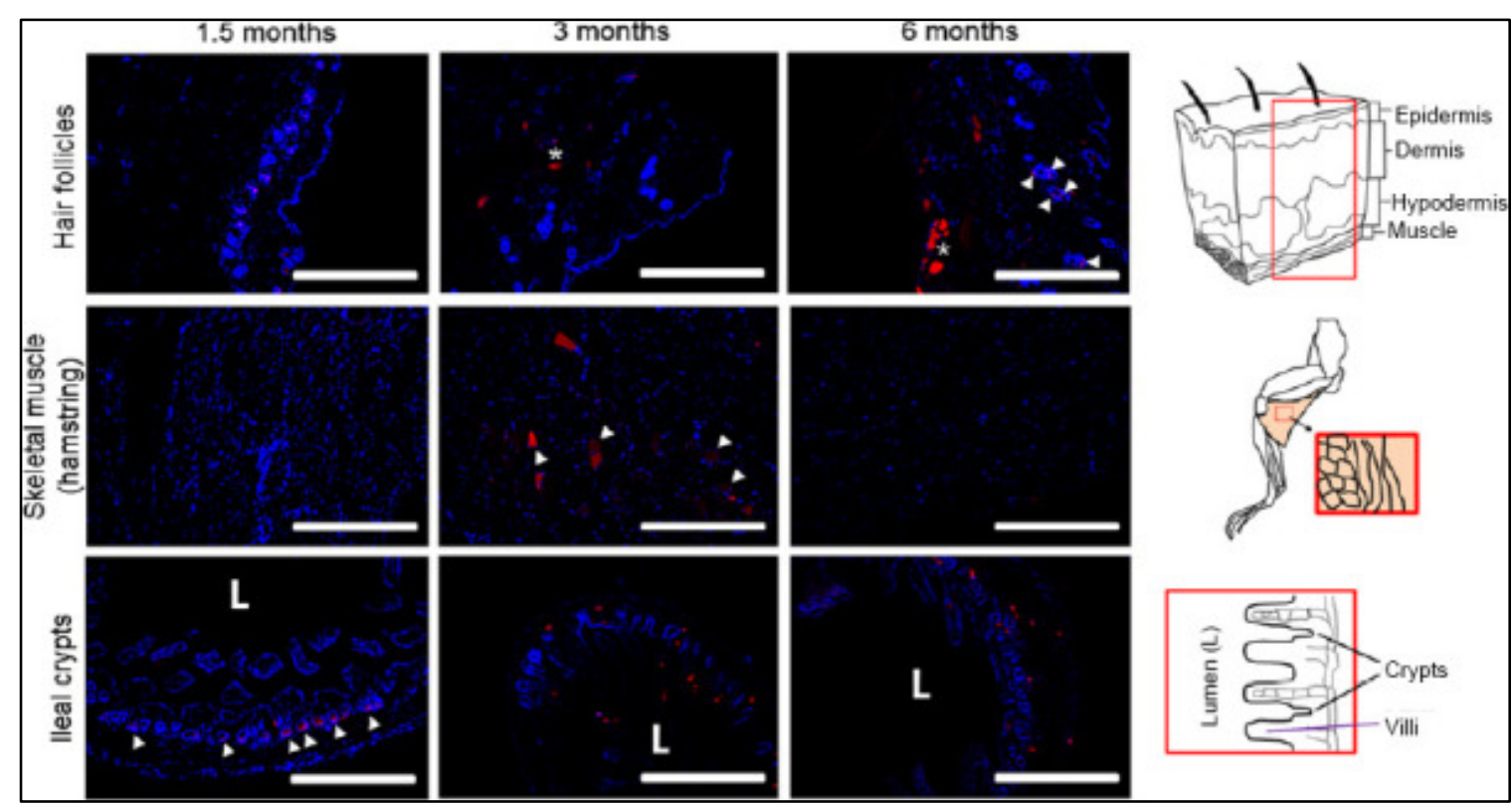

Figure A7 Nestin cell fate mapping in the periphery. Transgenic NesCreER $R^{T / R o s a-f l o x-S T O P-t d T o m a t o ~ m i c e ~ a t ~ 4-5 ~ m o n t h s ~ w e r e ~ f e d ~ t a m o x i f e n-l a d e n ~}$ chow and tissue har- vested at the indicated times following treatment initiation. The representative merged channel images were acquired from tissues that were fixed and Hoechst labeled. Cell lineage trac- ing is evidenced by tdTomato (red) and nuclei are indicated by Hoechst (blue) labeling. Note in hair follicles at 1.5 months after tamoxifen addition, the observed fluorescent signal is only found in hair shafts (no labeled cells). Labeled hair folli- cle cells observed at 6 months post-tamoxifen were confirmed K15negative. Red boxes displayed in diagrams on the right indicate region shown in the associated micrographs. Asterisk (*) indicates tdTomato ${ }^{+}$muscle fibers underlying skin. Arrow- heads highlight labeled cells and the ileal lumen (L) is indicat- ed [white scale bar: $400 \mu \mathrm{m}]$.

\section{$\underline{\text { Small Intestine }}$}

Nestin cell lineage tracing revealed positive labeling for ileal crypt cells at 1.5 months and absence at 3 and 6 months post-TAM treatment (Figure A7). Labeling was analogous to VEcadherin tracing for this tissue. Conversely, no labeling was observed for duodenal crypt cells, suggesting divergent mechanisms for these two tissues. Interestingly, a small mass of tdTomato-positive cells possessing irregular morphology (possible tumor) was noted in the ileum of a 3 month post-TAM mouse (Additional file 1). 


\section{$\underline{\text { Discussion }}$}

The non-endothelial tdTomato expression patterns observed in this study are likely due to differentiation events traced from endothelial cells. This is supported by previous work that examined the expression pattern of the VEcad-CreER ${ }^{T 2}$ mouse line used in this study (Monvoisin et al., 2006). Monvoisin et al. found that reporter gene expression by these mice is confined to endothelial cells following a 5-day intraperitoneal injection regimen of 4hydroxy-tamoxifen (4-OHT) when examined 7 days post-treatment. In addition, inspection of hematopoietic bone marrow cells revealed that $V E c a d-C r e E R^{T 2}$ mice exhibited very few reporter positive cells $(0.3 \%)$ compared to negative control mice $(0.2 \%)$ suggesting only a negligible contribution from these sources. This group also assessed skeletal muscle in adult mice (6-8 weeks of age) following 3-day tamoxifen injection at 2 days, 1 week and 6 weeks post-injection. No positive skeletal myocyte reporter expression, only endothelial cells (by morphology), was noted for any time point. This is consistent with our finding of tdTomato+ve skeletal muscle first appearing at 3 months post-treatment. It is important to note that the reporter used by Monvoisin and colleagues to examine $V E c a d-C r e E R^{T 2}$ mouse line expression utilized a $\beta$-galactosidase cassette (activated by CRE activity) residing in the ROSA26 locus and driven by native ROSA26 elements (Soriano, 1999). Our study utilizes a flox-STOP-tdTomato reporter also residing in the ROSA26 locus (Madisen et al., 2010). However, this reporter is driven by a CAG promoter that could exhibit differing expression (cell type, intensity and silencing) properties. We observe endothelial cell expression robustly in some tissues while it is markedly reduced in others. This could be due to properties conveyed by the CAG promoter and a possibility that not all endothelial cells exhibit the same expression profile in all tissues. Given the strong endothelial cell fidelity 
reported by Monvoisin et al. and numerous studies of VE-cadherin immunohistochemistry, the VEcad-CreER ${ }^{T 2}$ cassette portion of this dual transgenic system appears to be functioning as intended.

The robust labeling of large numbers of cerebellar granule neurons by VE-cadherin tracing was a notable finding. Unlike other regions of the brain, development of the cerebellum begins postnatally, which may represent an alternative development strategy for CGNs. It has been shown that despite elimination of canonical CGN precursors in the early postnatal mouse brain by irradiation and genetic approaches, CGNs are nevertheless generated at high levels (Wojcinski et al., 2017). Ponti et al. (2008) reported that cells previously classified as astroglia and interneuron progenitors are in fact distinct from neonatal canonical CGN progenitors and that in peripubertal rabbits they give rise to large numbers of adult-produced CGNs. A separate group of researchers has also presented evidence indicating that a Nestin-expressing progenitor pool is reprogrammed to contribute to CGN repopulation following acute depletion in postnatal mice (Wojcinski et al., 2017). Interestingly, we also observed Nestin ${ }^{+}$progenitors tracing to CGNs. Our study suggests that only a very small number of CGNs are regenerated (absent of injury) from a Nestinexpressing progenitor in adult mice. VE-cadherin ${ }^{+}$progenitor cells contribute to the vast majority of labeled CGNs, indicating at least two distinct pools of progenitor cells $\left(\mathrm{Nestin}^{+}\right.$and VE-cadherin $\left.{ }^{+}\right)$for the homeostasis of adult mouse cerebellum. These findings suggest an opportunity to deliver gene therapy intravenously for direct effects on CGN physiology.

Ependymal cells have been described as a source of CNS progenitor cells in the adult brain (Johansson et al., 1999) in addition to performing other important homeostatic 
functions such as cerebrospinal fluid production (Becht, 1920; Bruni et al., 1985). Here we report that the ependymal layer is sparsely labeled by VE-cadherin tracing throughout ventricular surfaces 3 months post-TAM (and including the choroid plexus just ventral to the cerebellum). Ependymal labeling was only visible in the choroid plexus adjacent to the cerebellum in the 1.5- and 6-month cohorts. We also observed intense Nestin tracing for the ubiquitous ventricle-lining ependymal layer throughout the adult mouse brain. These results suggest the possibility of a Nestin ${ }^{+} / \mathrm{VE}$-cadherin ${ }^{+}$choroid plexus progenitor in adult mice, which is consistent with an endothelial subtype.

This study also revealed VE-cadherin tracing to hair follicle cells. Examination by confocal microscopy revealed that a very small proportion of these cells were positive for a hair follicle stem cell marker (K15). This is a surprising finding, given that hair follicle stem cells are believed to be of mesenchymal origin. As such, $\mathrm{K} 15^{+}$stem cells would not be expected to be derived from a progenitor that expresses an endothelial marker. The highest level of labeling was observed 1.5 months post-TAM where most hair follicles had at least one positive cell per section; significantly reduced labeling was observed at 3 months and labeling was reduced to undetectable levels at 6 months. This implies a rapid turnover rate consistent with hair growth in mice. Unlike VE-cadherin, Nestin did not trace to hair follicle stem cells. We did, however, observe an enrichment of Nestin-traced hair follicle-associated capillaries.

Inspection of skeletal muscle from hamstring showed VE-cadherin tracing at 1.5 months post-TAM treatment followed by a peak in $\operatorname{tdTomato}^{+}$muscle fiber number at 3 months. No strongly positive myocytes were observed by 6 months and only faintly labeled fibers remained. This suggests that the signal resulting from the fusion of VE-cadherin-traced 
progenitors with myocyte cytosol had been diluted out through fusion with non-labeled progenitors after the 3 month post-TAM time point. We also observed Nestin tracing in hamstring at 3 months post-TAM (no labeling in the 1.5- or 6-month cohorts). Additionally, we found VE-cadherin and Nestin tracing to muscle underlying subcutaneous dorsal pelt. This indicates the possible existence of a Nestin ${ }^{+}$and VE-cadherin ${ }^{+}$skeletal muscle progenitor in adult mice. Previous investigations have reported the existence of skeletal muscle satellite cells in adult animals termed myoendothelial cells that express endothelial cell markers including VE-cadherin (Zheng et al., 2007). Whether these cells are of endothelial origin remains to be determined. Supporting the notion of endothelial-skeletal muscle transdifferentiation are reports of extravascular expression following intravenous delivery of transgenes. A group reported that intravenous delivery of adeno-associated virus (AAV9) containing CRISPR/CAS9 editing constructs, carried out in a canine model of Duchenne muscular dystrophy (Amoasii et al., 2018), resulted in successful editing of a mutant dystrophin gene in skeletal muscle. Interestingly, this group found an extremely high percentage of successfully edited/transduced skeletal myocytes at 2 months post-systemic AAV injection, even far surpassing what was observed in this study. Taken together, these findings imply that intravenous delivery of gene therapy could be undertaken to correct a defined mutation or provide a trophic factor to improve outcomes for skeletal muscle disease.

Investigation of VE-cadherin cell lineage tracing within the pancreas uncovered tdTomato labeling of islets. All islets examined at 1.5 months post-TAM contained tdTomato $^{+}$cells and $83 \%$ of islets at 3 months post-TAM displayed labeling. No labeling was evident at 6 months, suggesting complete turnover of labeled cells by that time. Confocal assessment confirmed that a small proportion (3\%) of tdTomato ${ }^{+}$islet cells were insulin- 
producing $\beta$ cells. No tdTomato-expressing cells were found to be glucagon ${ }^{+} \alpha$-cells. The presence of VE-cadherin-traced $\beta$ cells suggests the possible existence of a $\beta$ cell precursor cell in adult mice with endothelial origins. The evident decrease in tdTomato ${ }^{+}$islet cells over time provides support for the existence of regenerative mechanisms of $\beta$ cells in adult mice, which is currently a subject of controversy (Yu et al., 2016). The existence of a natural endothelial-derived $\beta$ cell progenitor could offer new intravenous therapy options for prolonged treatment of diabetes. In addition, this knowledge could inform strategies to stimulate $\beta$ cell regeneration in vivo.

Exocrine pancreatic acini were also found to contain tdTomato-labeled cells following VE-cadherin tracing. Only a few acini lobes per section exhibited the fluorescent marker, though when present, positive cells were grouped in close proximity. The highest abundance of labeled acini was found 1.5 months post-TAM, which decreased markedly 3 months after treatment. The 6-month group showed only rare and individual tdTomato $^{+}$acinar cells. This labeling pattern suggests the existence of an endothelial-derived progenitor and that near-complete regeneration of acini cells occurred over this 6-month period.

Renal tissue was also found to exhibit VE-cadherin tracing. Specifically, cells comprising the convoluted tubules within the renal cortex had pronounced labeling at 1.5 months post-TAM, with a gradual loss observed at 3 months and only a very faint signal detected at 6 months. The progenitor source for adult proximal convoluted tubule cells is still highly debated and our finding adds to a substantial body of evidence regarding extra-renal and atypical sources (Poulsom et al., 2001; Gupta et al., 2002; Sugimoto et al., 2006; Castrop, 2019). The observed fluorescent signal in this study likely does not arise from 
filtrate since the tissues were thoroughly flushed during multiple washes following sectioning onto slides. In addition, tdTomato labeling is completely absent from the renal medulla, excluding fluorescence contributed directly by filtrate cells in the loop of Henle or medullar collecting tubules. However, it cannot be ruled out that label-positive proximal convoluted tubule cells obtained tdTomato from early filtrate via their native protein-recovery mechanisms and thereby obtained cytosolic fluorescence independent of a transdifferentiation event. An alternative interpretation of this result could be that cell fusion events of proximal convoluted tubule cells with other tdTomato ${ }^{+}$cells decreased and that contributed cytoplasm was gradually turned over (similar to myocytes). Another possibility is that the weak signal at 6 months is due to activation of CRE by tamoxifen that has persisted. Further studies are needed to explore these possibilities as the prospect for renewal of these important cell types could be useful in the development of therapeutic strategies for the treatment of kidney disease.

Our assessment of the digestive tract revealed VE-cadherin cell lineage tracing of duodenal and ileal gland crypts. Labeling was confined to the base of the intestinal crypts and was robust at 1.5 months but disappeared 3 months after TAM treatment began. This suggests a rapid turnover of these cells, which is consistent with that of cells found within a highly proliferative epithelial tissue. A weaker fluorescent signal was observed for more mature cells emanating from the crypts that form the intestinal villi. Interestingly, we did not observe any tdTomato labeling within colonic crypts, which suggests a different etiology in adult mice. We also found that Nestin traced to Ileal crypts at 1.5 months post-TAM (and disappeared by 3 months) but did not trace to the duodenum suggesting divergent origins for these adult tissues. 


\section{Conclusion}

There is evidence of tissue homeostasis by actively dividing stem cells and one or more pools of quiescent stem cells working in concert to maintain cell replacement needs (Li and Clevers, 2010; Blanpain and Fuchs, 2014). In such cases, it is possible that the selector of the donating quiescent progenitor is the tissue environment rather than the cell type classification of the progenitor itself. For example, a growing body of evidence indicates that some quiescent brain regions possess the ability to foster the differentiation of transplanted progenitor cells from varied sources into functional neurons (Lindvall et al., 1989; Volkman and Offen, 2017). Moreover, experimental ablation of one type of progenitor can cause restoration by another type (Rompolas et al., 2013; Blanpain and Fuchs, 2014). If indeed this form of plasticity occurs broadly in adult mammals, endothelial cells would represent the most readily available cell type for "recruitment" as a progenitor across tissues. Evidence presented in this study supports the notion of an endothelial population that transdifferentiates into other embryonically distinct cell types and which is also a substantial contributor to adult mammalian tissue homeostasis. Confirmation of such a finding would offer new insights into the pathology of human disease and present potential therapeutic avenues.

\section{Limitations of the CreERT2/Rosa-floxed-STOP-tdTomato System}

- a) Cell fusion events cannot be completely ruled out. However, Hoechst labeling of nuclei did not reveal any multinucleated cells (with the exception of myocytes) by conventional or confocal microscopy. In addition, previous studies involving bone marrow-derived stem cell transplantation (Alvarez-Dolado et al., 2003) did not reveal 
cell fusion events for any of the tdTomato ${ }^{+}$cells observed in this study, indicating that tdTomato-expressing cells are likely not derived from fusion events with hematopoietic cells (e.g., B-cells).

- b)The genomic location (Rosa26) of the reporter cassette, in concert with the exogenous promoter employed (CAG promoter-driven-tdTomato), dictates the celltype expression pattern of tdTomato following the initial activation from flox-STOP removal by $\mathrm{CreER}^{\mathrm{T} 2}$. Therefore, genomic editing by activated $\mathrm{CreER}^{\mathrm{T} 2}$ may have occurred in a given cell without the production of a fluorescent signal if the Rosa26 locus or CAG promoter is silenced by that cell type.

- $\quad$ c) CRE activity can result in two outcomes. Correct editing creates a tdTomato sequence with the removal of a stop-codon-containing cassette and the subsequent expression of a functional fluorescent reporter. However, CRE activity can also result in translocation of cut DNA fragments to other locations in the genome or other rearrangements that do not produce a functional reporter. In these instances, cell lineage tracing mechanisms would be activated but not observed.

- d) Promoter silencing is a reported cellular response to numerous constitutive promoter systems (e.g., CMV, CAG) (Choi et al., 2005; Xia et al., 2007; Herbst et al., 2012). Reporter gene silencing could also confound the disappearance of the tdTomato signal at later time points. Other approaches would be necessary to determine if a loss in signal is due to cell turnover or CAG promoter silencing.

- $\quad$ e) The observation of fewer reported tracing events could be the result of cell death. Double-stranded cuts directed by CRE in target cells initiate DNA repair systems. If 
repairs are not made correctly or in a timely fashion, cells are susceptible to apoptosis. In addition, cell death could be directed by the immune system against tdTomato-derived peptides presented in MHC class I complexes. However, this prospect is unlikely, given that tdTomato is observed in some tissues in both VEcadherin and Nestin mouse lines at 6 months post-TAM.

- $\quad$ f) Contributions by extracellular vesicles emitted from tdTomato-expressing cells cannot be determined in this system. It is possible that extracellular vesicles containing tdTomato are released by edited/labeled cells and accumulated in target cells, thereby leading to fluorescence in the target cell that is not associated with a transdifferentiation event. This is an inherent limitation of the Rosa-floxed-STOPtdTomato system. 
Additional Figures

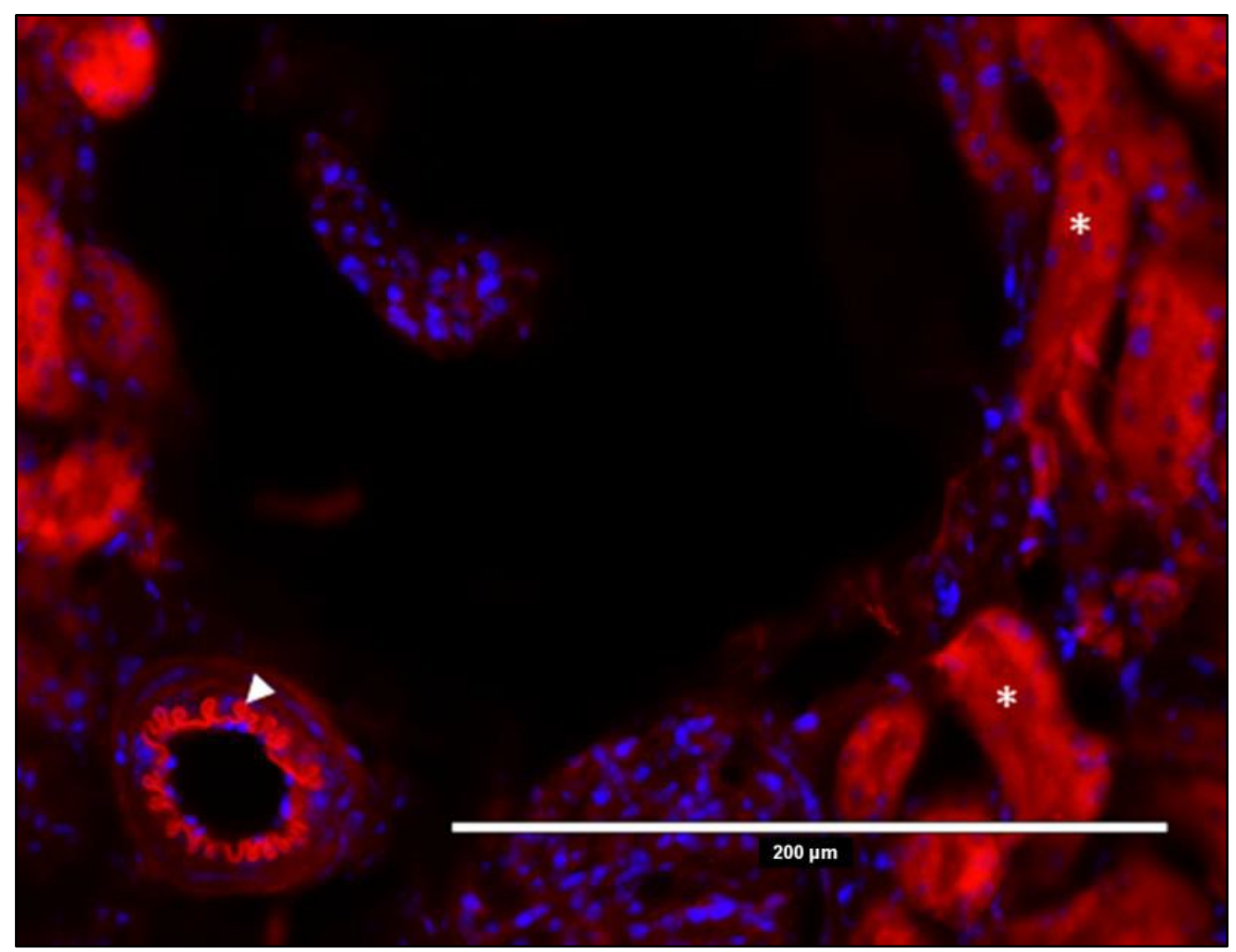

Figure AS1. Prototypical arterial endothelila cell expression of dTomato following VE-cadherin cell-lineage tracing in real tissue. Renal tissue was harvested from VEcad$\mathrm{CreER}^{T 2} / \mathrm{Rosa}$-flox-STOP-tdTomato transgenic mice 1.5 months post-tamoxifen treatment initiation. Tissue samples were fixed, cryosectioned $(15 \mu \mathrm{m})$ onto slides, stained with Hoechst dye (nuclear label) and then mounted with coverslips. The displayed imagewas captured by an EVOS M7000 conventional fluorescence microscopy system. The merged image shows tdTomato (red) and Hoechst (blue) channels. Arrowhead indicates tdTomato+ endothelial cells of the internal elastic lamina of a renal glomerulus arteriole. Asterisks (*) show tdTomato+ convoluted tubules also present in this micrograph. 


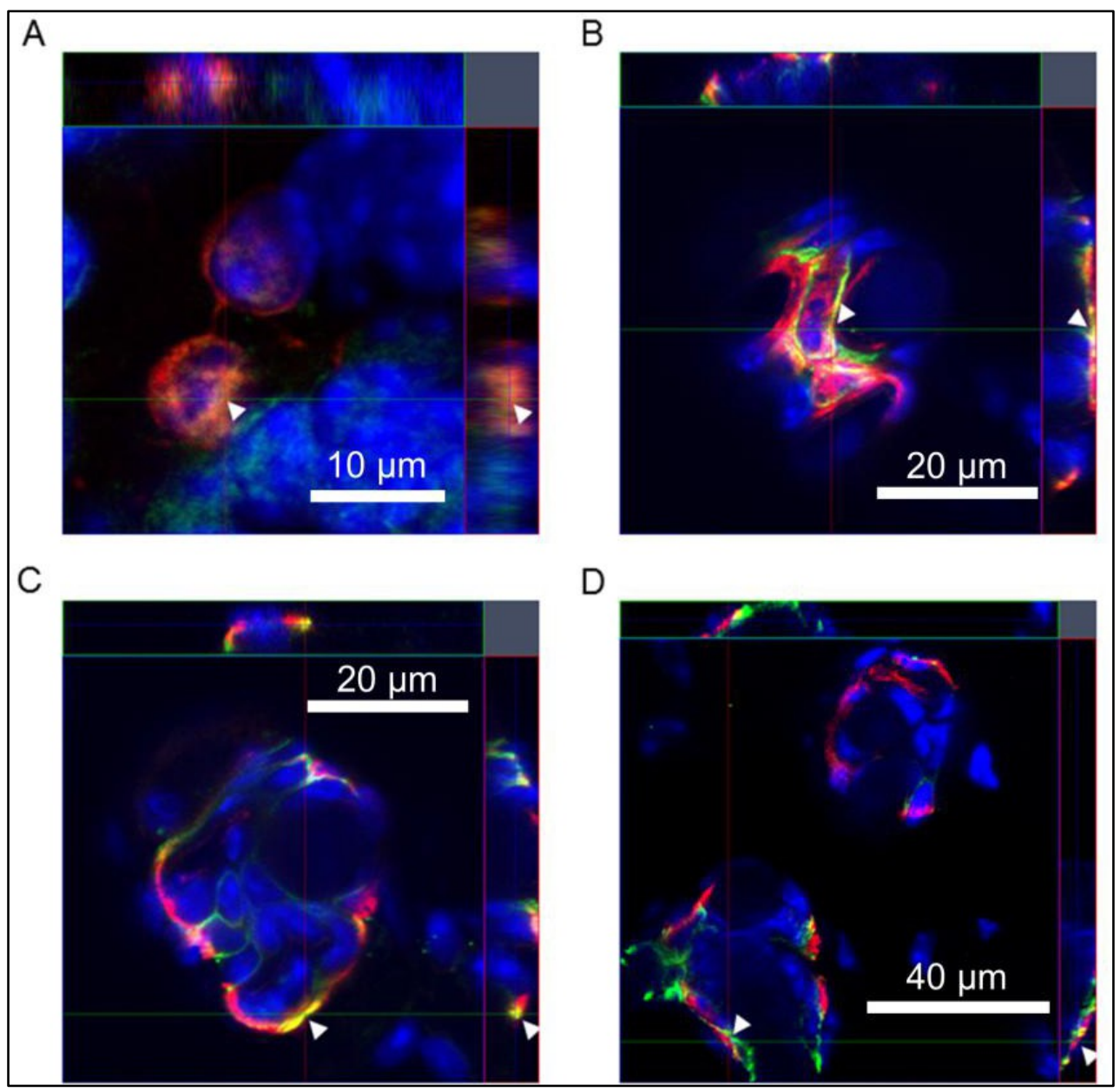

Figure AS2. Confocal microscopy orthogonal analysis of VE-cadherin-traced cerebellargranule neurons and hair follicle cells presented in Figure A3. (A) NeuN (green) /tdTomato (red) co-labeling (arrowheads) by IHC confirmed for cerebellar granule neurons through orthogonal reconstruction from confocal image stacks. $(B, C)$

Rare hair follicular cells exhibit K15 (green)/tdTomato (red) co-localization (arrowheads). (D) The vast majority of tdTomato hair follicle cells do not exhibit colabeling with K15 (green) despite often being in very close proximity. Images captured using a Zeiss Laser Scanning Confocal Microscope Meta 510 system. 


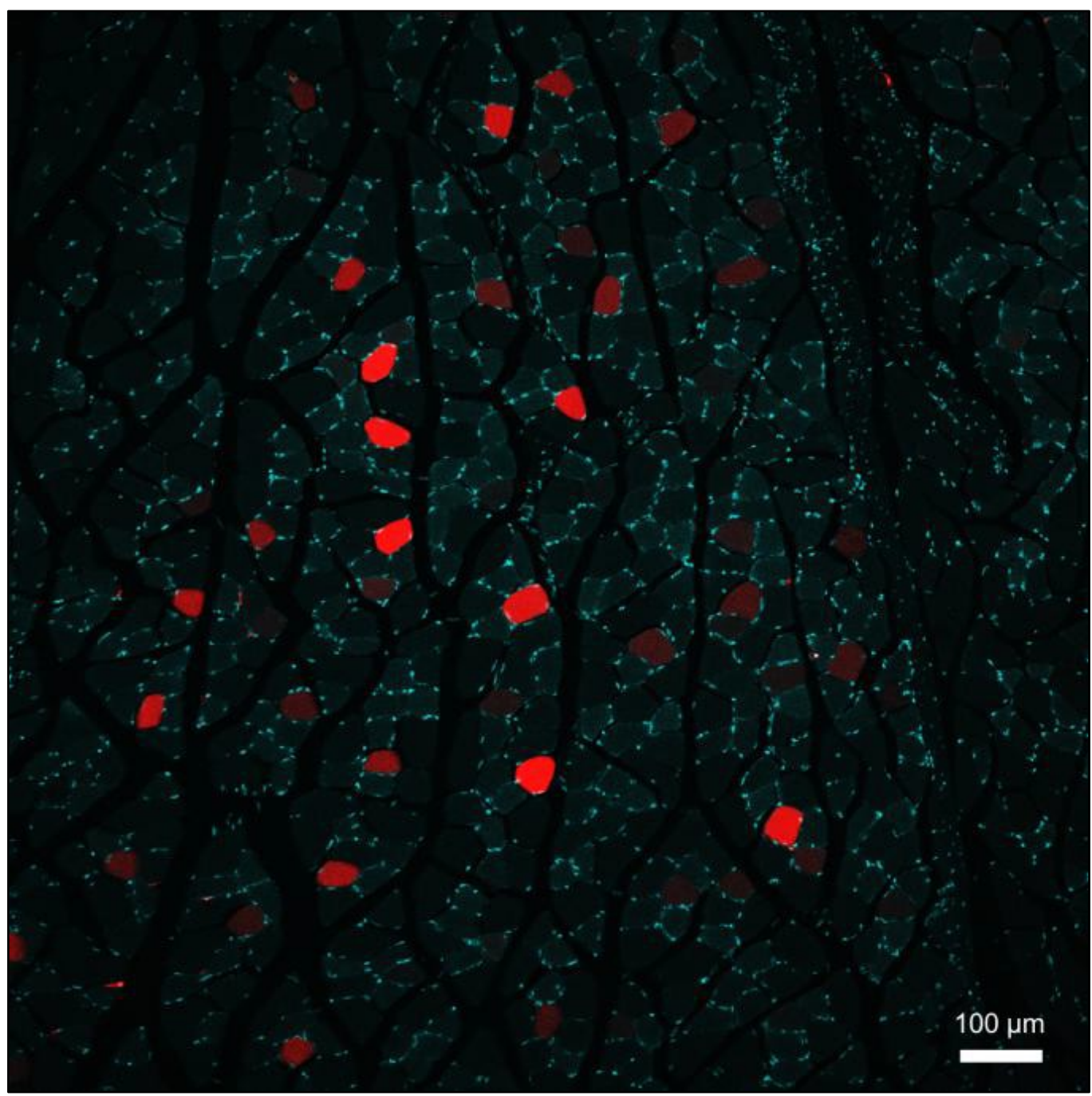

Figure AS3. Examination of skeletal myocytes following VE-cadherin cell lineage tracinghigh resolution confocal image. Hamstring tissue was harvested from $\mathrm{VEcad}$ $\mathrm{CreER}^{T 2} /$ Rosa-flox-STOP-tdTomato transgenic mice 3 months post-tamoxifen treatment initiation. Fixed samples were sectioned $(15 \mu \mathrm{m})$ onto slides, labeledwith Hoechst dye, mounted with coverslips, and imaged using an Olympus FV3000 Laser Scanning Confocal Microscope system. The displayed image was obtained from a series of $10 \mu \mathrm{m}$ z-stacked planes compressed into a maximum intensity projection image showing tdTomato (red) and Hoechst (blue) channels. 


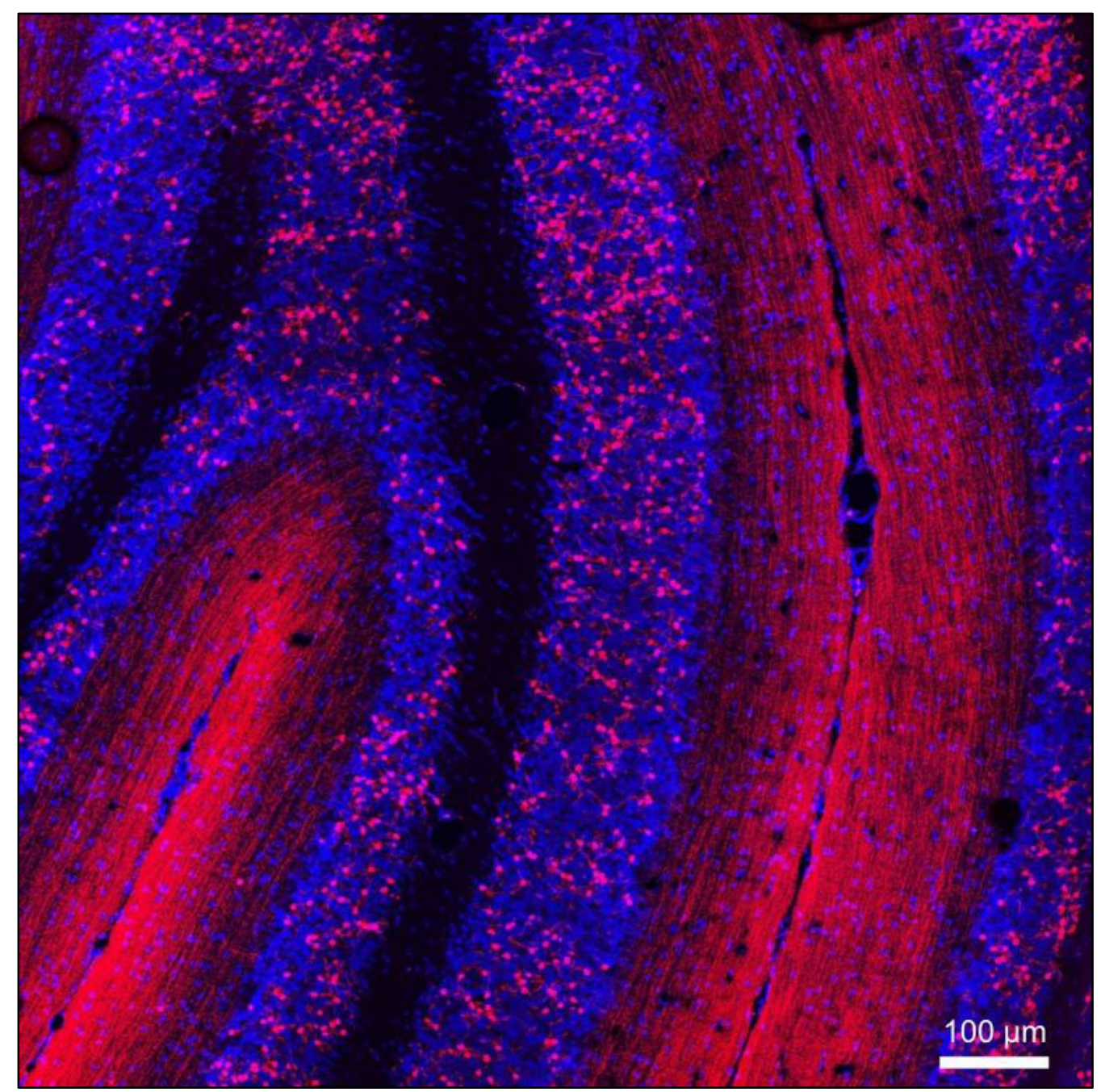

Figure AS4. VE-cadherin-traced cerebellar granule neurons confocal microscopy high resolution tiled image.Tissue was harvested from $\mathrm{VEcad}-\mathrm{CreER} \mathrm{R}^{T 2} / \mathrm{Rosa}$-flox-STOPtdTomato transgenic mice 3 months post-tamoxifen treatment initiation. The cryosectioned tissue $(15 \mu \mathrm{m})$ was fixed, labeled with Hoechst dye, and mounted with a coverslip. A tiled image was captured for $10 \mu \mathrm{m}$ z-stacked planes and stitched using a Zeiss 880 Airy Scan system. Hoechst dye (blue) and tdTomato (red) channels are displayed in the maximum intensity projection image shown. 


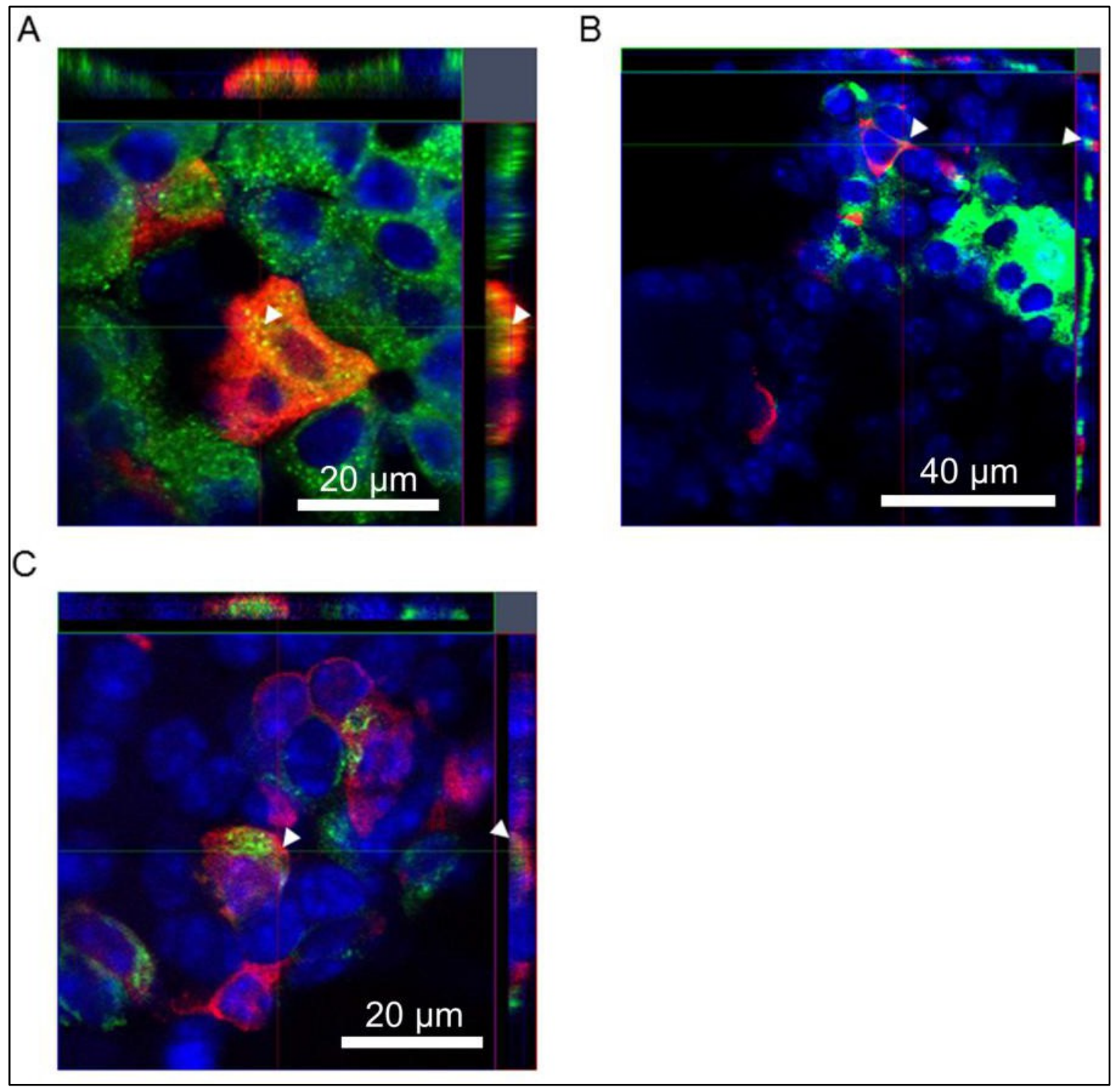

Figure AS5 Confocal microscopy orthogonal analysis of VE-cadherin-traced pancreaticislet cells presented in Figure A5. (A) Immunohistochemistry for insulin/tdTomato-co-labeled cell (arrowhead) assessed by orthogonal reconstruction from confocal stacked images. (B) Almost all tdTomato positive islet cells examined are insulin (green)-negative despite very close physical proximity. (C) No glucagon (green)/tdTomato co-labeled cells were observed in any pancreatic section examined. Arrowheads indicate a point of assessment. A Zeiss Laser Scanning Confocal Microscope Meta 510 system was used to capture and analyze the images shown. 


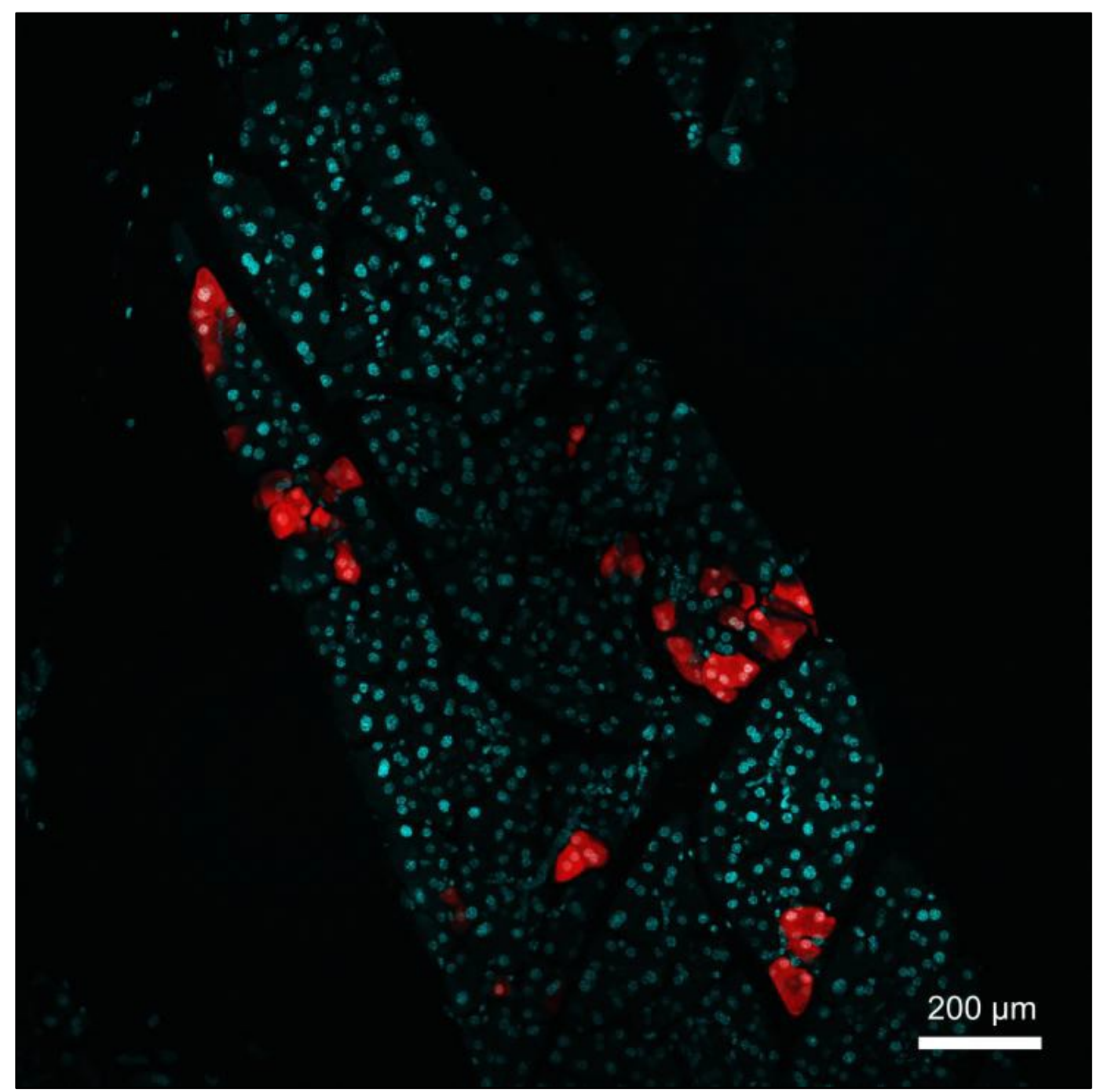

Figure AS6 VE-cadherin cell lineage tracing high-resolution confocal image of pancreatic acini.Pancreatic tissue was harvested from $\mathrm{VEcad}-\mathrm{CreER} \mathrm{R}^{T 2} / \mathrm{Rosa}$-flox-STOPtdTomato transgenic mice 3 months post-tamoxifen treatment initiation. Tissue samples were fixed, cryosectioned $(15 \mu \mathrm{m})$ onto slides, stained with Hoechst dye and then mounted with coverslips. The displayed image was captured by an Olympus FV3000

Laser Scanning Confocal Microscope system from $10 \mu \mathrm{m}$ z-stacked planes and represented as a maximum intensity projection. The merged image shows tdTomato (red) and Hoechst (blue) channels. 


\section{References}

Albright JE, Stojkovska I, Rahman AA, Brown CJ, Morrison BE (2016) Nes- tinpositive/SOX2-negative cells mediate adult neurogenesis of nigral dopa- minergic neurons in mice. Neurosci Lett 615:50-54.

Altman J (1969) Autoradiographic and histological studies of postnatal neuro- genesis. IV. Cell proliferation and migration in the anterior forebrain, with special reference to persisting neurogenesis in the olfactory bulb. J Comp Neurol 137:433-457.

Altman J, Das GD (1965) Autoradiographic and histological evidence of postna- tal hippocampal neurogenesis in rats. J Comp Neurol 124:319-335.

Alvarez-Dolado M, Pardal R, Garcia-Verdugo JM, Fike JR, Lee HO, Pfeffer K, Lois C, Morrison SJ, Alvarez-Buylla A (2003) Fusion of bone-marrow-derived cells with Purkinje neurons, cardiomyocytes and hepatocytes. Nature 425:968- 973.

Amoasii L, Hildyard JCW, Li H, Sanchez-Ortiz E, Mireault A, Caballero D, Harron R, Stathopoulou T-R, Massey C, Shelton JM, Bassel-Duby R, Piercy RJ, Olson EN (2018) Gene editing restores dystrophin expression in a canine model of Duchenne muscular dystrophy. Science 362:86-91.

Battiste J, Helms AW, Kim EJ, Savage TK, Lagace DC, Mandyam CD, Eisch AJ, Miyoshi G, Johnson JE (2007) Ascll defines sequentially generated lineage-re- stricted neuronal and oligodendrocyte precursor cells in the spinal cord. De- velopment 134:285-293.

Bayer SA, Yackel JW, Puri PS (1982) Neurons in the rat dentate gyrus granular layer substantially increase during juvenile and adult life. Science 216:890-892. Becht FC (1920) Studies on the cerebrosp inal fluid. American Journal of Physiology-Legacy Content 51:1-125.

Bernanke DH, Markwald RR (1982) Migratory behavior of cardiac cushion tis- sue cells in a collagen-lattice culture system. Dev Biol 91:235-245. 
Bifari F, Decimo I, Pino A, Llorens-Bobadilla E, Zhao S, Lange C, Panuccio G, Boeckx B, Thienpont B, Vinckier S, Wyns S, Bouche A, Lambrechts D, Giug- liano M, Dewerchin M, Martin-Villalba A, Carmeliet P (2017) Neurogenic radial glia-like cells in meninges migrate and differentiate into functionally integrated neurons in the neonatal cortex. Cell Stem Cell 20:360-373.

Blanpain C, Fuchs E (2014) Stem cell plasticity. Plasticity of epithelial stem cells in tissue regeneration. Science 344:1242281.

Bruni JE, Del Bigio MR, Clattenburg RE (1985) Ependyma: normal and patho- logical. A review of the literature. Brain Res 356:1-19.

Camenisch TD, Molin DGM, Person A, Runyan RB, Gittenberger-de Groot AC, McDonald JA, Klewer SE (2002) Temporal and distinct TGFbeta ligand re- quirements during mouse and avian endocardial cushion morphogenesis. Dev Biol 248:170-181.

Castrop H (2019) The role of renal interstitial cells in proximal tubular regenera- tion. Nephron 141:265-272.

Chen PY, Qin L, Baeyens N, Li G, Afolabi T, Budatha M, Tellides G, Schwartz MA, Simons M (2015) Endothelial-to-mesenchymal transition drives athero- sclerosis progression. J Clin Invest 125:4514-4528.

Choi KH, Basma H, Singh J, Cheng P-W (2005) Activation of CMV promot- er-controlled glycosyltransferase and beta-galactosidase glycogenes by butyr- ate, tricostatin A, and 5-aza-2'-deoxycytidine. Glycoconj J 22:63-69.

de Lange FJ, Moorman AFM, Anderson RH, Männer J, Soufan AT, de Gier-de Vries C, Schneider MD, Webb S, van den Hoff MJB, Christoffels VM (2004) Lineage and morphogenetic analysis of the cardiac valves. Circ Res 95:645- 654.

Ding L, Morrison SJ (2013) Haematopoietic stem cells and early lymphoid pro- genitors occupy distinct bone marrow niches. Nature 495:231-235.

Donato AJ, Morgan RG, Walker AE, Lesniewski LA (2015) Cellular and Molecu- lar Biology of Aging Endothelial Cells. J Mol Cell Cardiol 89(Pt B):122-135 
Dusart P, Fagerberg L, Perisic L, Civelek M, Struck E, Hedin U, Uhlén M, Trégouët D-A, Renné T, Odeberg J, Butler LM (2018) A systems-approach reveals human nestin is an endothelial-enriched, angiogenesis-independent intermediate filament protein. Sci Rep 8:14668.

Trégouët D-A, Renné T, Odeberg J, Butler LM (2018) A systems-approach reveals human nestin is an endothelial-enriched, angiogenesis-independent intermediate filament protein. Sci Rep 8:14668.

Eisenberg LM, Markwald RR (1995) Molecular regulation of atrioventricular val- vuloseptal morphogenesis. Circ Res 77:1-6.

Gupta S, Verfaillie C, Chmielewski D, Kim Y, Rosenberg ME (2002) A role for extrarenal cells in the regeneration following acute renal failure. Kidney Int 62:1285-1290.

Heimark RL, Degner M, Schwartz SM (1990) Identification of a Ca2(+)-depen- dent cell-cell adhesion molecule in endothelial cells. J Cell Biol 110:1745-1756.

Herbst F, Ball CR, Tuorto F, Nowrouzi A, Wang W, Zavidij O, Dieter SM, Fessler S, van der Hoeven F, Kloz U, Lyko F, Schmidt M, von Kalle C, Glimm H (2012) Extensive methylation of promoter sequences silences lentiviral transgene expression during stem cell differentiation in vivo. Mol Ther 20:1014-1021.

Hussaini SMQ, Jun H, Cho CH, Kim HJ, Kim WR, Jang MH (2013) Heat-induced antigen retrieval: an effective method to detect and identify pro- genitor cell types during adult hippocampal neurogenesis. J Vis Exp doi: 10.3791/50769.

Itkin T, Gur-Cohen S, Spencer JA, Schajnovitz A, Ramasamy SK, Kusumbe AP, Ledergor G, Jung Y, Milo I, Poulos MG, Kalinkovich A, Ludin A, Kollet O, Shakhar G, Butler JM, Rafii S, Adams RH, Scadden DT, Lin CP, Lapidot T (2016) Distinct bone marrow blood vessels differentially regulate haematopoi- esis. Nature 532:323-328.

Johansson CB, Momma S, Clarke DL, Risling M, Lendahl U, Frisén J (1999) Identification of a neural stem cell in the adult mammalian central nervous system. Cell 96:25-34.

Kretzschmar K, Watt FM (2012) Lineage tracing. Cell 148:33-45. 
Li L, Clevers H (2010) Coexistence of quiescent and active adult stem cells in mammals. Science 327:542-545.

Li Y, Lui KO, Zhou B (2018) Reassessing endothelial-to-mesenchymal transition in cardiovascular diseases. Nat Rev Cardiol 15:445-456.

Lindvall O, Rehncrona S, Brundin P, Gustavii B, Astedt B, Widner H, Lindholm T, Björklund A, Leenders KL, Rothwell JC, Frackowiak R, Marsden D, Johnels B, Steg G, Freedman R, Hoffer BJ, Seiger A, Bygdeman M, Strömberg I, Olson L (1989) Human fetal dopamine neurons grafted into the striatum in two pa- tients with severe Parkinson's disease. A detailed account of methodology and a 6-month follow-up. Arch Neurol 46:615-631.

Madisen L, Zwingman TA, Sunkin SM, Oh SW, Zariwala HA, Gu H, Ng LL, Palmiter RD, Hawrylycz MJ, Jones AR, Lein ES, Zeng H (2010) A robust and high-throughput Cre reporting and characterization system for the whole mouse brain. Nat Neurosci 13:133-140.

Menezes JR, Smith CM, Nelson KC, Luskin MB (1995) The division of neuronal progenitor cells during migration in the neonatal mammalian forebrain. Mol Cell Neurosci 6:496-508.

Merrell AJ, Stanger BZ (2016) Adult cell plasticity in vivo: trans-differentiation is back in style. Nat Rev Mol Cell Biol 17:413-425.

Michalopoulos GK, Barua L, Bowen WC (2005) Transdifferentiation of rat hepatocytes into biliary cells after bile duct ligation and toxic biliary injury. Hepatology 41:535-544.

Mjaatvedt CH, Lepera RC, Markwald RR (1987) Myocardial specificity for initi- ating endothelial-mesenchymal cell transition in embryonic chick heart cor- relates with a particulate distribution of fibronectin. Dev Biol 119:59-67.

Mokrý J, Ehrmann J, Karbanová J, Cízková D, Soukup T, Suchánek J, Filip S, Kolár Z (2008) Expression of intermediate filament nestin in blood vessels of neural and nonneural tissues. Acta Medica (Hradec Kralove) 51:173-179. 
Monvoisin A, Alva JA, Hofmann JJ, Zovein AC, Lane TF, Iruela-Arispe ML (2006) VEcadherin-CreERT2 transgenic mouse: a model for inducible recombina- tion in the endothelium. Dev Dyn 235:3413-3422.

Moonen JR, Lee ES, Schmidt M, Maleszewska M, Koerts JA, Brouwer LA, van Kooten TG, van Luyn MJ, Zeebregts CJ, Krenning G, Harmsen MC (2015) Endothelial-tomesenchymal transition contributes to fibro-proliferative vas- cular disease and is modulated by fluid shear stress. Cardiovasc Res 108:377- 386.

Oh M, Nör JE (2015) The perivascular niche and self-renewal of stem cells. Front Physiol $6: 367$.

Palmer TD, Ray J, Gage FH (1995) FGF-2-responsive neuronal progenitors reside in proliferative and quiescent regions of the adult rodent brain. Mol Cell Neu- rosci 6:474-486.

Ponti G, Peretto P, Bonfanti L (2008) Genesis of neuronal and glial progenitors in the cerebellar cortex of peripuberal and adult rabbits. PLoS One 3:e2366.

Poulsom R, Forbes SJ, Hodivala-Dilke K, Ryan E, Wyles S, Navaratnarasah S, Jef- fery R, Hunt T, Alison M, Cook T, Pusey C, Wright NA (2001) Bone marrow contributes to renal parenchymal turnover and regeneration. J Pathol 195:229- 235.

Rahman AA, Lai NK, Albright JE, Urquhart PE, Webb AR, Morrison BE (2017) Nigral dopaminergic neuron replenishment in adult mice through VE-cad- herin-expressing neural progenitor cells. Neural Regen Res 12:1865-1869.

Rompolas P, Mesa KR, Greco V (2013) Spatial organization within a niche as a determinant of stem-cell fate. Nature 502:513-518.

Soriano P (1999) Generalized lacZ expression with the ROSA26 Cre reporter strain. Nat Genet 21:70-71.

Sugimoto H, Mundel TM, Sund M, Xie L, Cosgrove D, Kalluri R (2006) Bone-marrowderived stem cells repair basement membrane collagen defects and reverse genetic kidney disease. Proc Natl Acad Sci U S A 103:7321-7326. 
Suzuki S, Namiki J, Shibata S, Mastuzaki Y, Okano H (2010) The neural stem/ progenitor cell marker nestin is expressed in proliferative endothelial cells, but not in mature vasculature. J Histochem Cytochem 58:721-730.

Tamplin OJ, Durand EM, Carr LA, Childs SJ, Hagedorn EJ, Li P, Yzaguirre AD, Speck NA, Zon LI (2015) Hematopoietic stem cell arrival triggers dynamic remodeling of the perivascular niche. Cell 160:241-252.

Tang Z, Wang A, Yuan F, Yan Z, Liu B, Chu JS, Helms JA, Li S (2012) Differenti- ation of multipotent vascular stem cells contributes to vascular diseases. Nat Commun 3:875.

Tarlow BD, Pelz C, Naugler WE, Wakefield L, Wilson EM, Finegold MJ, Grompe M (2014) Bipotential adult liver progenitors are derived from chronically in- jured mature hepatocytes. Cell Stem Cell 15:605-618.

Volkman R, Offen D (2017) Concise review: mesenchymal stem cells in neurode- generative diseases. Stem Cells 35:1867-1880.

Wojcinski A, Lawton AK, Bayin NS, Lao Z, Stephen DN, Joyner AL (2017) Cer- ebellar granule cell replenishment postinjury by adaptive reprogramming of Nestin+ progenitors. Nat Neurosci 20:1361-1370.

Wurmser AE, Nakashima K, Summers RG, Toni N, D'Amour KA, Lie DC, Gage FH (2004) Cell fusion-independent differentiation of neural stem cells to the endothelial lineage. Nature 430:350-356.

Xia X, Zhang Y, Zieth CR, Zhang SC (2007) Transgenes delivered by lentiviral vector are suppressed in human embryonic stem cells in a promoter-depen- dent manner. Stem Cells and Dev 16:167-176.

Yu K, Fischbach S, Xiao X (2016) Beta cell regeneration in adult mice: controver- sy over the involvement of stem cells. Curr Stem Cell Res Ther 11:542-546.

Zerlin M, Levison SW, Goldman JE (1995) Early patterns of migration, morpho- genesis, and intermediate filament expression of subventricular zone cells in the postnatal rat forebrain. J Neurosci 15:7238-7249. 
Zheng B, Cao B, Crisan M, Sun B, Li G, Logar A, Yap S, Pollett JB, Drowley L, Cassino T, Gharaibeh B, Deasy BM, Huard J, Peault B (2007) Prospective identification of myogenic endothelial cells in human skeletal muscle. Nat Biotechnol 25:1025-1034. 
APPENDIX B 


\section{Repurposing Drugs to Treat Heart and Brain Illness}

Citation: Cantrell, M, S, Soto-Avellaneda A, Wall, J, D, Ajeti, A, D, Morrison, B, E, McDougal, O,M, Warner, L, R. (2021). Repurposing drugs to treat heart and brain illness. Pharmaceuticals, 14(6), 573. https://doi.org/10.3390/ph14060573

\section{$\underline{\text { Abstract }}$}

Drug development is a complicated, slow and expensive process with high failure rates. One strategy to mitigate these factors is to recycle existing drugs that have viable safety profiles and have gained Food and Drug Administration approval following extensive clinical trials. Cardiovascular and neurodegenerative diseases are quite difficult to treat, and there exist few effective therapeutics, necessitating the development of new, more efficacious drugs. Recent scientific studies have led to mechanistic understanding of heart and brain disease progression, which has led researchers to assess myriad drugs for their potential as pharmacological treatments for these ailments. The focus of this review is to survey strategies for selection of drug repurposing candidates and provide representative case studies where drug repurposing was used to discover therapeutics for cardiovascular and neurodegenerative disorders, wherein anti-inflammatory drugs have profound side-effects during chronic use and therefore new alternatives are needed.

\section{$\underline{\text { Introduction }}$}

Drug development is a long, labor-intensive process with no guarantee of success. On average, it takes 10 years and 2.6 billion dollars to develop a new drug, with success rates averaging only about $12 \%$ [1]. One way to mitigate the barriers of drug development is to repurpose Food and Drug Administration (FDA) approved drugs for the treatment of different diseases. Drug repurposing is the practice of finding new uses for existing drugs at any stage of development. The benefit of drug repurposing is that the 
risk of failure is significantly lower than drug development, which permits more effective use of resources to optimize drug efficacy for treatment of the desired ailment. Much of the primary research regarding repurposed drug safety, mechanism and dosage has already been completed at the time the drug was first studied [2]. For example, a drug that demonstrated efficacy in animal models with low side effects but failed in human clinical trials to perform its intended purpose, may be a good candidate for use as a therapeutic for a different disease. Drugs with potential to be repurposed have progressed through many of the steps required to meet regulatory safety and potency benchmarks, allowing for an accelerated and abbreviated path toFDA approval. A few representative examples of successful drugs that have been repurposed include amantadine, aspirin, mecamylamine, minoxidil and tamoxifen (Table B1) [3]. Repurposed drugs are generally screened by computer search algorithm from databases of therapeutics that may or may not have been sufficiently effective in providing treatment for the pathology that served as their original target. For example, some drugs lack sufficient drug-like qualities to warrant further investigation for treatment of an intended illness, but modification of the molecular scaffold or functional groups attached to the drug template may afford attributes and activity suitable for therapeutic viability. The therapeutic index for a repurposed drug requires investigation into dosage recommendations for maximal benefit to counter the illness, while minimizing detrimental side-effects to the patient. 
Table B1. Examples of repurposed drugs

\begin{tabular}{|c|c|c|c|}
\hline Drug & Original purpose & Repurposed use & Reference \\
\hline Amantadine & Parkinson's disease & $\begin{array}{c}\text { Influenza A, } \\
\text { COVID-19 } \\
\text { (hypothesized) }\end{array}$ & {$[4,5]$} \\
\hline Aspirin & $\begin{array}{c}\text { Inflammation, pain } \\
\text { relief }\end{array}$ & Anti-platelet & {$[6]$} \\
\hline Mecamylamine & Hypertension & Tourette's syndrome & {$[7]$} \\
\hline Minoxidil & Hypertension & Hair loss & {$[8]$} \\
\hline Tamoxifen & Cancer & Leishmaniasis & {$[9]$} \\
\hline
\end{tabular}

\section{Technology to repurpose drugs}

Computational strategies for determining potential drug repurposing candidates are a great starting place for researchers to find drugs that have previously been on the market to take to new clinical trials. Multiple computational strategies have emerged to identify potential candidates for drug repurposing. Of these, four strategies have gained popularity in recent years and will be discussed in brief. These strategies are side effect similarity mapping, genome-wide association studies (GWAS), small-molecule peptideinfluenced drug repurposing (SPIDR), and computational high throughput screening (HTS) screening [10,11].

In side-effect similarity mapping, existing drugs are categorized by their side effects using the Unified Medical Language System (UMLS) ontology for medical symptoms. Developed by Campillos et al. (2008), 746 marketed drugs were organized according to their listed phenotypic side effect similarities rather than a chemical similarity, and 20 of these marketed drugs were confirmed through in vitro assays to indicate that side effect similarity may be indicative of common protein targets. Using this strategy, prediction of the likelihood that two drugs had the same target based on 
their side effects was demonstrated [12]. This study describes a process by which drugs marketed for different targets could be identified as having potential for repurposing.

GWAS identify single nucleotide polymorphisms (SNPs) and their associated phenotypes for individuals with a given disease. GWAS is performed by genotyping individuals which share a common disease and determining whether a genetic variant is shared to statistical significance among this population. Experimental validation by chromatin precipitation is then performed and variants associated by phenotypic effects [13]. This technique has been used to identify many novel variant to trait associations in a wide variety of diseases such as macular degeneration, anorexia nervosa, depression, coronary artery disease and diabetes mellitus, and can be used to determine novel drug targets as well [14]. For example, GWAS have been used to identify SNPs for Parkinson's disease (PD) and determined that estradiol may protect dopaminergic neurons in PD. This supported previous evidence that estradiol may be protective against PD because men are more likely to be diagnosed [15]. The neuroprotective role of estradiol was later confirmed in in vivo studies. At present, estradiol is still not an approved treatment for PD, but efficacy studies are ongoing [2,16,17]. Another example of GWAS being useful for determining novel drug targets was the identification of a gene encoding a disintegrin and metalloproteinase with thrombospondin motifs TS7

(ADAMTS7), a gene implicated in coronary artery disease (CAD) for its presence in angiographic $\mathrm{CAD}$ patients and role in smooth muscle cell migration, a finding that supports the idea of genetically inherited CAD $[18,19]$. Using GWAS, it is possible to identify new potential targets based on SNPs. 
SPIDR is a recently developed computational screening technique to identify drugs that can target a specific receptor by looking at its peptide ligand conformational space [20]. This method was integrated into the free program DockoMatic v 2.1, making this process accessible to lower resourced institutions [21]. This technique works by first utilizing the genetic algorithm managed peptide mutant screening (GAMPMS) to screen for peptide mutants with high binding affinity to the receptor, and then this population of peptides is moved over to the SimSearcher utility to search for the top small molecules analogous to the peptide population for binding to the isoform [20]. SPIDR was developed and used to find 12 small molecules computationally predicted to bind with high affinity to a nicotinic acetylcholine receptor (nAChR) isoform associated with Alzheimer's and PD, by looking at alpha conotoxin MII peptide analogs that would bind favorable to the receptor isoform specifically [20]. Following this publication, a qualitative assay to detect dopamine release by ligand action on nAChRs was developed with the intent to assess bioactivity of molecules that may act on different nAChR isoforms for drug development. This assay was developed using luminescence-based assay and validated with known nAChR binders acetylcholine, nicotine and cytisine [22]. This assay is intended to be used to test the small molecules found in the SPIDR development publication for $\mathrm{AAChR}$ activity as an in vitro validation for the SPIDR methodology.

Additionally, small molecule compound screens can be used to identify drugs amenable to being repurposed. Computational programs such as AutoDock Vina are often used to screen molecular library databases like ZINC, chEMBL, pubchem, DrugBank, Chem Spider, and others [23]. Computational screens, in silico, are an ideal 
starting point for any drug discovery project to narrow down a list of molecules that may bind your target with high affinity. However, wet lab experimentation needs to be conducted, which can be done with commercially available libraries of drugs using in vitro cell-based assays. Researchers study whether a drug being tested induces a phenotypic effect in cell-based assays, which are usually performed in cell culture or on a model organism whereas a target-based screen can be performed in vitro using techniques like ELISA, Surface Plasmon Resonance (SPR) or Nuclear Magnetic Resonance (NMR) spectroscopy. In this article we will briefly discuss strategies for repurposing drugs and examples of drugs that are being repurposed for treatment of cardiovascular disease and neurodegenerative disorders. Specifically, we will cover those conditions that affect both cardiovascular and neurodegenerative disease such as inflammatory pathways, as these conditions connect heart and brain disease.

\section{Heart and brain interconnectedness in disease}

There are currently few therapeutics for cardiovascular and neurodegenerative diseases that provide suitable efficacy and minimal side effects, creating ideal conditions for drug repurposing. Not only are cardiovascular and neurodegenerative diseases difficult to treat, but treatment options remain limited, and many drugs do not cease progression nor achieve remission of the disease state. Considerable progress has been made in recent years to understand the molecular determinants of cardiovascular disease, which encompasses a wide variety of disorders such as endothelial dysfunction, atherosclerosis and pulmonary arterial hypertension, and neurodegenerative disorders, such as Parkinson's and Alzheimer's [24,25] Cardiovascular disease and neurodegenerative disorders share a common trait in that inflammation is a component to 
the pathogenic process. Targeting inflammatory processes with repurposed drugs can have a multitude of beneficial effects for patients with diseases of both the heart and brain. Currently, repurposed drugs are being used in studies associated with two cardiovascular related diseases that include endothelial dysfunction and pulmonary arterial hypertension. As for neurodegenerative diseases, repurposed drugs are being studied for the treatment of autophagy and neuroinflammation.

\section{Targeting endothelial dysfunction in cardiovascular disease}

Cardiovascular diseases including atherosclerosis, coronary artery disease and arrhythmias that eventually lead to myocardial infarction and/or stroke. Ischemic events such as these are the leading cause of death in the world [26]. Pharmacological intervention of cardiovascular diseases is provided by statins, beta-blockers, and angiotensin converting enzyme inhibitors. In more advanced cases, surgical intervention may be necessary. The medications for cardiovascular disease target high cholesterol and low-density lipoprotein levels, and hypertension. There is an emerging field of research to discover small molecule inhibitors for alternative pathways aimed at the disruption of heart disease progression [27]. There is a robust market for pharmaceutical companies to synthesize drugs to treat disorders, but the potential for repurposing already FDAapproved drugs constitutes a desirable alternative to discovery since these medications have been deemed safe enough for patient use and thus make clinical trials for novel use non-trivial.

The endothelium is characterized by the cells of the tissue lining the various organs of the body, including the arteries and lymphatic system. The endothelium plays a 
major role in blood flow and constriction by facilitating the synthesis and degradation of vasodilating factors, such as nitric oxide (NO), arachidonic acid metabolites and reactive oxygen species (ROS) [28]. Endothelial dysfunction occurs when the endothelium fails to function properly. Namely, that the endothelium dysfunctions in the formation of vasodilating factors. Endothelial dysfunction is especially important in cardiovascular disease because it is a leading contributor to cardiac events. Endothelial dysfunction is a component of atherosclerosis, which is characterized by the hardening and narrowing of the arterial walls, and hyperlipidemia, or high lipid concentrations in the blood.

Three drugs investigated for their potential to treat diseases of endothelial dysfunction that will be discussed here include colchicine, which has been traditionally used to treat gout, methotrexate, an immunosuppressant and chemotherapy drug, and Tocilizumab (brand name Actrema), an immunosuppressant used to treat severe rheumatoid arthritis.

\section{Colchicine}

Colchicine is a secondary metabolite from the plants Gloriosa superba and Colchicum autumnale, that is known to be toxic when ingested [29]. Its current approved use is to treat gout, an inflammatory form of arthritis common in people with high levels of uric acid in their blood. Due to its anti-inflammatory properties, colchicine is currently under investigation as a potential endothelial dysfunction drug. Endothelial dysfunction is a characteristic of cardiovascular disease that leads to an increase in macrophages, $\mathrm{T}$ lymphocytes and growth factors that contribute to atherosclerotic lesion formation associated with atherosclerosis [30-33]. The same inflammation inhibition mechanism 
that permits colchicine to be effective against gout, may also be applicable to the treatment of patients with early stages of atherosclerosis [34,35].

Colchicine prevents inflammation by binding to tubulin, preventing tubulin polymerization and function (see Figure B1) [18]. As a result, colchicine halts mitotic cells in metaphase. Colchicine also concentrates in neutrophils and prevents chemotaxis via the release of crystal-derived chemotactic factor, and inhibits monosodium urate induced loss of myeloid inhibitory C-type lectin-like receptor in neutrophils and interleukin-8 formation [36] Myeloid inhibitory C-type lectin-like receptor and interleukin-8 are important in immune homeostasis and inflammatory response [37,38].

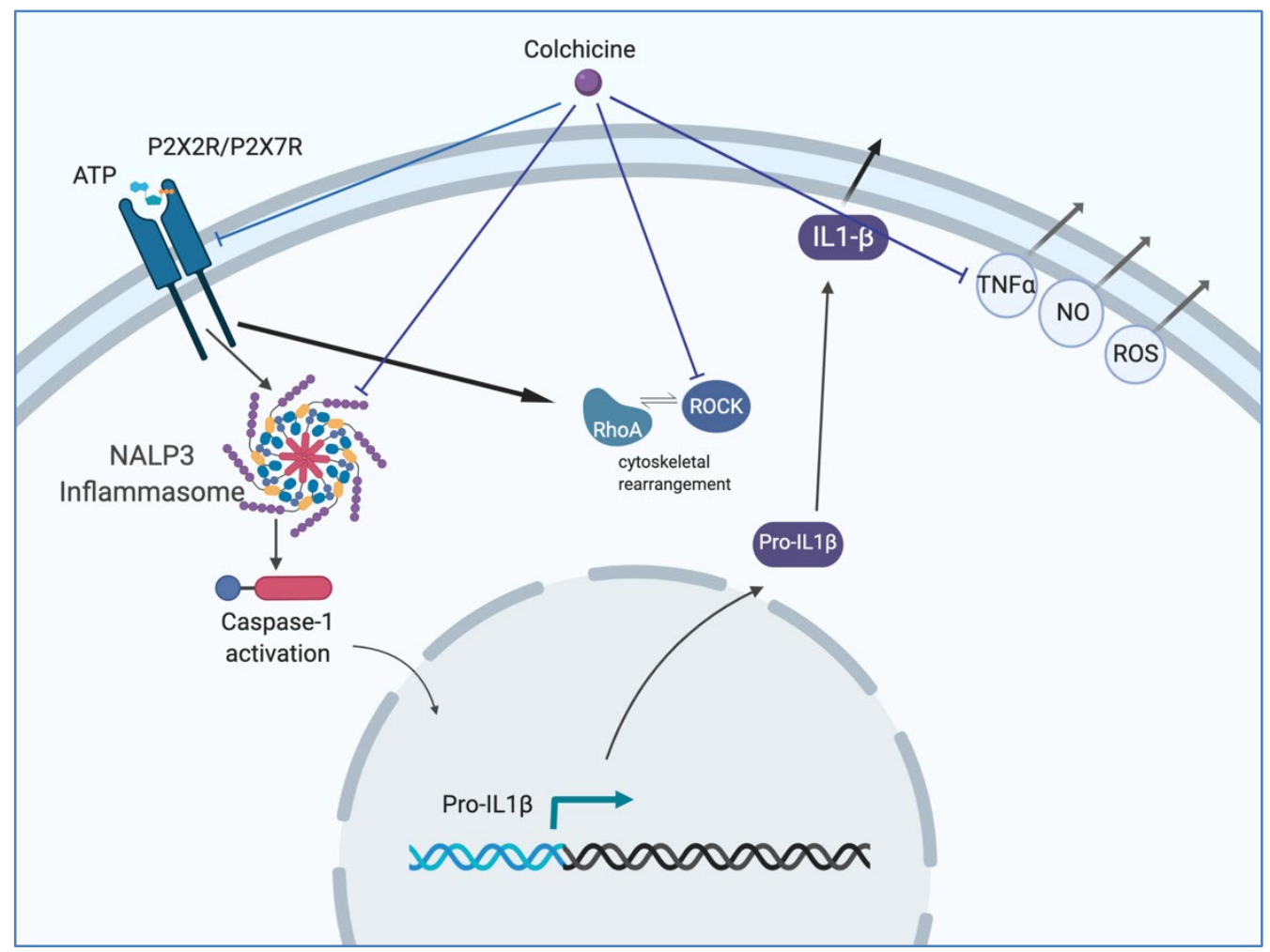

Figure B1. Inhibitory pathway of colchicine.Colchicine inhibits activation of purinergic P2X2/P2X7 receptors and blocks cation uptake and subsequent proinflammatory signaling cascades without affecting cell death. Colchicine also inhibits the NALP3 inflammasome pathway, the Rho/ROCK pathway via cytoskeleton rearrangement, and inhibits release of ROS, NO and TNFa. Figure 1 was created using BioRender.com (adapted from [36] . 
Cell adhesion is the process by which cells adhere to one another through the action of proteins, electrostatic interactions, and hydrophobic interactions. Adhesion is important because cells can use physical contact with one another to communicate via signal transduction. Colchicine interferes with cell adhesion by inducing the shedding of neutrophil adhesion molecules, which are important in neutrophil function [36]. These neutrophil adhesion molecules include selectins and counter receptors which partake in signal transduction. Selectins are single-chain transmembrane glycoproteins organized by leukocyte (L), endothelial (E) and platelet/endothelial (P) selectins. They are characterized by their similar homology in both sequence, structure and sugar ligands. Colchicine reduces endothelial expression of selectins by acting on leukocytes in coronary artery disease [35].

Nidorf et al. reviewed ongoing clinical trial results and experimental evidence that supported the use of colchicine as an anti-inflammatory drug with promising efficacy toward the treatment of atherosclerosis [34]. First, they describe the mechanisms by which colchicine acts on the various inflammatory pathways including the production of pro-inflammatory cytokines, such as interleukin (IL)-1ß, reduction of platelet leukocyte interactions that lead to atherothrombosis and suppressing the growth of fibroblasts and osteophytes. At lower doses, colchicine was found to have no reported side effects on patients with liver and/or kidney disease [34]. The authors concluded that colchicine could be repurposed for treatment of inflammation associated with atherosclerosis.

\section{Methotrexate}

Aminopterin, a precursor drug to methotrexate, has been used to treat childhood 
leukemia, dating back to the late 1940's and early 1950's. It was noted that the drug interfered with connective tissue proliferation in patients undergoing leukemia treatment, which led to studies into the effect of aminopterin on rheumatic disease [39]. A study in 1951 by Gubner et al. demonstrated that aminopterin was effective in alleviating signs and symptoms of the disease associated with rheumatoid arthritis, psoriasis and psoriatic arthritis. The synthesis of aminopterin proved to be a barrier to drug studies, inspiring creation of methotrexate, which was easier to synthesize, but equally as efficacious as aminopterin (Figure B2). In the 1960's, the National Institute of Health reported that methotrexate was effective at treating rheumatoid arthritis and psoriatic arthritis. However, the rheumatologist community at the time maintained a fidelity for corticosteroids to treat rheumatic syndromes, which stifled the use of methotrexate. Further, and larger studies were conducted that established irrefutable evidence that methotrexate was effective in the treatment of rheumatoid arthritis [40]. 
<smiles>CCC[C@H](CC(C)=O)NC(=O)c1ccc(NCC2=NC3C(=O)NC(N)=NC3N=C2)cc1</smiles><smiles>CN(Cc1cnc2nc(N)nc(N)c2n1)c1ccc(C(=O)N[C@@H](CCC(=O)O)C(=O)O)cc1</smiles><smiles>Nc1nc(N)c2nc(CNc3ccc(C(=O)N[C@@H](CCC(=O)O)C(=O)O)cc3)cnc2n1</smiles>

Figure B2. Structures of folic acid and its analogs methotrexate and aminopterin 
Methotrexate is a synthetic disease-modifying anti-rheumatic drug that is a structural analog of folic acid [25]. Methotrexate was originally used as a cancer drug but has recently been investigated for its use in inflammation associated with endothelial dysfunction. In a study of 673 patients, methotrexate was used for the treatment of rheumatoid arthritis, and was determined that under proper dosing and use, it could be a beneficial drug to treat arthritis with $1.7 \%$ adverse side effect frequency and $0.15 \%$ mortality [41]. Methotrexate was identified as a good candidate for use to treat inflammatory diseases, including endothelial dysfunction.

Methotrexate exerts a multitude of biochemical and biological perturbations when administered to patients. While the exact or primary mechanism of action is not completely understood, multiple modes of activity have been characterized that reveal how methotrexate functions (Figure B3). First, methotrexate diminishes the immune response by increasing the rate of $\mathrm{T}$ cell apoptosis. Methotrexate binds dihydrofolate reductase, causing an increase in nitric oxide synthase uncoupling through the prevention of the conversion of dihydrobiopterin to tetrahydrobiopterin (FigureB3a). Second, methotrexate suppresses inflammation and the immune response by promoting the release of adenosine. Metabolites of methotrexate inhibit aminoimidazole-4-carboxamide ribonucleotide (AICAR) transformylase, causing an increase in the concentration of intracellular AICAR and thus more adenosine release (Figure B3b). Third, methotrexate increases the expression of long intergenic non-coding RNA p21, a mediator and regulator of a variety of pro-apoptotic processes (Figure B3c) [42]. 


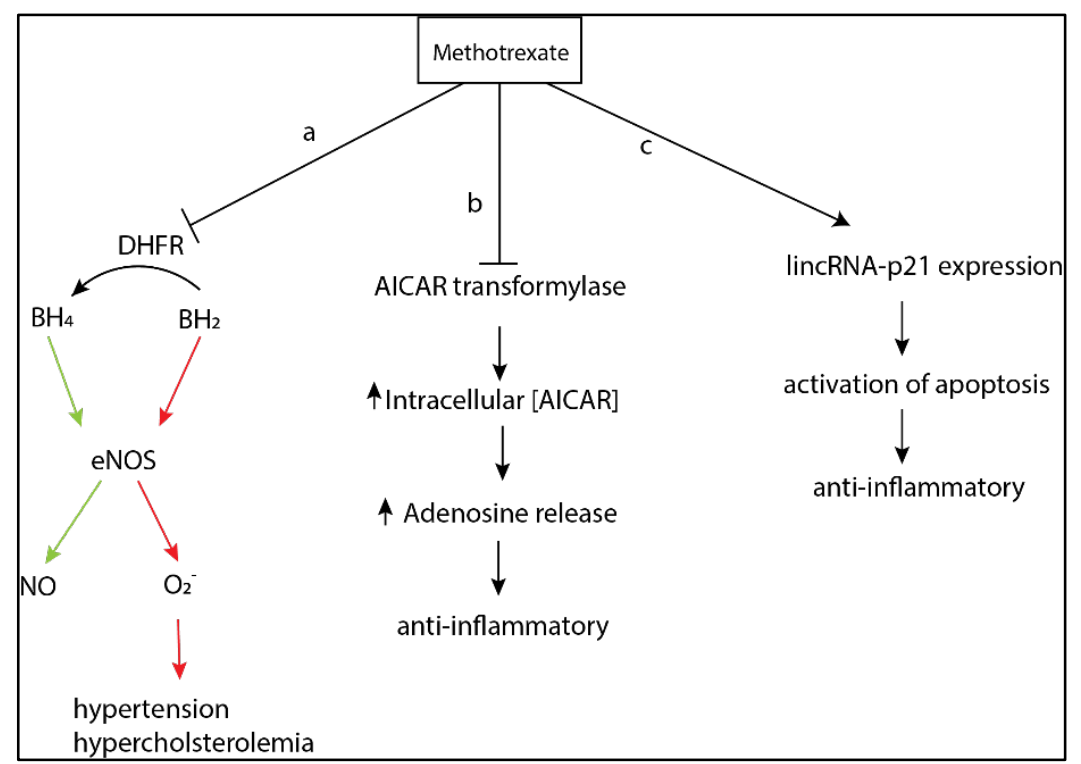

Figure B3. Metabolic pathways of methotrexate. a) Methotrexate inhibits dihydrofolate reductase (DHFR) and prevents conversion of dihydrobiopterin $\left(\mathrm{BH}_{2}\right)$ to tetrahydrobiopterin ( $\left.\mathrm{BH}_{4}\right)$, leading to uncoupling of NO synthase. b) Methotrexate inhibits AICAR transformylase, leading to increased adenosine levels and subsequent anti-inflammatory responses. c) Methotrexate stimulates lincRNAp21 expression, leading to increased apoptotic gene expression and subsequent antiinflammatory responses.

There is increasing evidence that the 5'-adenosine monophosphate-activated protein kinase (AMPK) is beneficial to the endothelium by protecting against apoptosis, oxidative stress and increases NO production in the endothelium, which reduces cholesterol efflux activity [25]. Stimulation of AMPK as a treatment for endothelial dysfunction is under investigation. One study showed that application of methotrexate on perivascular adipose tissue from Sprague-Dawley rats increased AMPK activity under basal and inflammatory conditions when treated with a negative control, palmitic acid [25]. A positive control using aminoimidazole-4-carboxamide ribonucleotide, a known AMPK agonist, was also able to stimulate AMPK activity [25]. This study similarly showed that methotrexate stimulated adiponectin mRNA expression and suppressed the 
pro-inflammatory molecules nuclear factor kappa $\mathrm{B}(\mathrm{NF \kappa B}) \mathrm{p}-65$, tumor necrosis factor alpha (TNF- $\alpha$ ), and interleukin-6 (IL-6) activity [25]. At the time of this review, only studies of methotrexate on perivascular adipose tissue taken from sacrificed mice and in vitro studies in cell culture have been performed, necessitating investigation into more physiologically relevant in vivo model systems.

Contrarily, other studies have indicated that methotrexate may contribute to vascular endothelial dysfunction by causing endothelial damage [43]. Methotrexate is a direct competitor with folic acid for the active site of human serum albumin, and indeed folic acid supplements are often provided in conjunction with methotrexate to prevent folic acid deficiency [44]. A study performed in Wistar rats, by Sankrityayan in 2016, showed that when administered with methotrexate, ex vivo vascular reactivity in the aorta was significantly reduced [43]. Vascular reactivity is the phenomenon responsible for both vasoconstriction and vasodilation, and those responses to various stimuli implicated in vasculature. Methotrexate has also been implicated in the reduction of NO, leading to increased oxidative stress and hindering of bioavailability of tetrahydrobiopterin by oxidizing it to dihydrobiopterin in the aorta $[45,46]$. This leads to hypertension, hyperlipidemia and induces endothelial dysfunction, because tetrahydrobiopterin is necessary as a cofactor required for synthesis of NO [47].

There is contradicting evidence for methotrexate use as an endothelial dysfunction drug. On one hand, there is data that suggests methotrexate reduces inflammation, while on the other hand, methotrexate appears to lead to endothelial dysfunction. Therefore, more studies must be performed to determine the true mechanism of action for 
methotrexate on the vascular endothelium if it is to be used to treat endothelial dysfunction. There are currently 2140 clinical trials listed under the National Library of Medicine's clinical trial database with methotrexate being evaluated for treatment of everything from ectopic pregnancy, to schizophrenia, to alopecia, and cancer. It appears that in many pathologies where inflammation is a principal concern, or where immunomodulation is of utility, methotrexate may provide benefit.

\section{Tocilizumab}

Tocilizumab (TCZ) is an IL-6 inhibitor and recombinant monoclonal antibody used in rheumatoid arthritis treatment since obtaining FDA approval in 2010 [48]. TCZ binds IL-6 and blocks JAK/STAT signaling to prevent the production of proinflammatory molecules $[48,49]$. It is currently being investigated for the treatment of endothelial dysfunction due to its anti-inflammatory properties. This is because RA is often listed as a co-morbidity in approximately $39-50 \%$ of atherosclerosis-related deaths, with endothelial dysfunction presented in many RA patients [50]. One hypothesis is that the release of networks of chromatin and granules (NETs) into the extracellular space, a common occurrence in both RA and endothelial function. TCZ was found to alleviate symptoms in endothelial dysfunction in a human clinical study [49].

\section{Targeting pulmonary arterial hypertension}

Pulmonary arterial hypertension $(\mathrm{PAH})$ is a relatively rare condition, affecting approximately 10-52 people per million population, characterized by the narrowing of the pulmonary arteries [51,52]. Left untreated, pulmonary arterial hypertension leads to the buildup of pressure on the right side of the heart when blood vessels in the lungs are 
diseased, causing the arteries to become increasingly narrow with time, and right-sided heart failure, often resulting in premature death $[53,54]$. While the direct cause of pulmonary arterial hypertension is unknown, a set of factors including liver disease, HIV infection, intravenous drug use, autoimmune disorders, and others are suspected to be culpable. For pulmonary arterial hypertension, there are several drugs under investigation for use in PAH in clinical trials and are discussed in detail [55]. Of these, two drugs, Anakinra and Ubenimex, target inflammatory processes specifically.

\section{Anakinra}

Anakinra was approved for use in rheumatoid arthritis by the FDA in 2001. Anakinra is currently under investigation and has undergone Phase I clinical trial studies for novel use in PAH [55,56]. Anakinra, an interleukin-1 (IL-1) receptor protein antagonist analogue, is recombinantly expressed in E. coli and is administered via subcutaneous injection. The proposed mechanism of action for $\mathrm{PAH}$, done in mice, is such that anakinra blocks perivascular macrophage recruitment in pulmonary artery smooth muscle cells [57]. In an inflammatory response pathway, IL-1b binds to IL-1 receptor (IL-1R) and recruits the myeloid differentiation primary response protein 88 (MyD88) and induces IL-1 synthesis via NFאB activation. In this study, the goal was to investigate whether this pathway played a role in the progression of PAH.57 First, it was determined that in both the lung tissue taken of patients with PAH as well as mice with PAH, there were increased expression levels of both IL-1R and MyD88. In IL-1R-/- and MyD88-/- mice, severity of PAH was not significantly different between the two but were significantly lower in severity than hypoxic wild-type mice. Hypoxic wild-type mice treated with anakinra $(20 \mathrm{mg} / \mathrm{kg}$ per day) were shown to have lower severity in 
PAH symptoms, namely, right ventricular systolic presser and hypertrophy, than untreated mice. It was also observed than in both IL-1R-/- and MyD88-/- hypoxic mice as well as wild-type mice treated with anakinra, there was no significant fold change in IL$1 \mathrm{~b}$ levels when compared to wild-type untreated mice with hypoxia over 48 hours. These findings suggest that anakinra is a good potential treatment for PAH in that IL-1b is not having to be expressed as an emergency measure for PAH. Furthermore, the pilot Phase I clinical study (clinicaltrials.gov: NCT03057028) done with treatment of PAH by anakinra found promising results by inducing IL-1 blockade.

\section{Ubenimex}

Ubenimex, also known as bestatin, is not currently approved for use by the FDA for any disease; however, it has undergone Phase II clinical trials for potential treatment of PAH [55]. Its original usage was for leukemia [58]. Ubenimex is a leukotriene A4 hydrolase (LTA4H) inhibitor, thought to block leukotriene B4 formation, thought to be involved in PAH pathogenesis via pulmonary artery smooth muscle cell proliferation [55,59]. However, results from the Phase II clinical trial are as yet unpublished.

\section{Modulating Autophagy in Neurodegeneration}

Neurodegenerative disorders are devastating, irreversible conditions that are characterized by the loss of neurons in the brain. In many cases, the onset of neurodegeneration associated with diseases including Parkinson's and Alzheimer's, is sporadic with no known genetic or environmental cause [60,61]. Given the absence of a causative agent, there are no disease-modifying treatments available, and existing drugs serve only to treat symptoms in an attempt to prolong the effects of disease progression 
[62]. There exits an important need for treatment options that prevent, slow or stop the progression of neurodegenerative disorders.

Neurodegenerative disorders can affect different areas of the nervous system, and cause patients to exhibit different symptoms depending on the disease; however, many share important characteristics outside the gradual loss of neurons over time. For example, Alzheimer's Disease, Parkinson's Disease, Amyotrophic Lateral Sclerosis and Huntington's Disease all exhibit protein aggregate pathology in the form of neurofibrillary tangles, Lewy bodies, inclusion bodies, and mutant huntingtin aggregates, respectively [63]. It is believed that these aggregates are toxic and play a role in neuronal death. Neurodegenerative disorders are also commonly associated with aberrant activation of the innate and adaptive immune systems in the central nervous system. Currently, significant research efforts are focused on targeting the mechanism by which these aggregates are removed by cell, namely autophagy. Alternatively, efforts also include studying the role inflammation plays in these disorders [64].

Autophagy is a cellular process responsible for maintaining protein homeostasis in the cell. While there is more than one mechanism of autophagy, macro-autophagy is the most studied and best understood type. For this reason, we will focus on macroautophagy (hereafter referred to as autophagy). In autophagy, cytoplasmic materials such as proteins, metabolites, and organelles are engulfed by membrane-bound vesicles known as autophagosomes. These autophagosomes are then trafficked to a lysosome, which then fuses with the autophagosome and is then degraded and released [63].

Because a hallmark of neurodegenerative diseases is the accumulation of protein 
aggregates, and autophagy is known to clear large aggregates of protein, autophagy is thought to be a potential target for a disease-modifying treatment. Furthermore, dysfunction in autophagy and its regulation have been implicated in many common neurodegenerative diseases. In Parkinson's Disease, mutations in PTEN-Induced Kinase 1 (PINK1) and Parkin, an E3 ubiquitin ligase involved in mitochondrial turnover, are known to disrupt the autophagic degradation of mitochondria leading to stress-induced mitochondrial dysfunction and cell death. Additionally, mutations in Park9 disrupt acidification in the lysosome [65]. In AD, reductions in expression of Beclin 1, a protein involved in the induction of autophagy, and Rab5, an endosomal and lysosomal regulator, have been implicated in the progression of the disease [65]. Finally, in Huntington's disease, huntingtin protein is involved in various aspects of autophagy, such as cargo recognition, endosomal and lysosomal regulation, and vesicular trafficking, and its canonical polyglutamine tract mutation has been shown to interfere with these regular functions (of autophagy) (Figure B4) [65]. Felodipine and lonafarnib are two examples of drug repurposing candidates that have undergone study for their activity to target autophagy. 


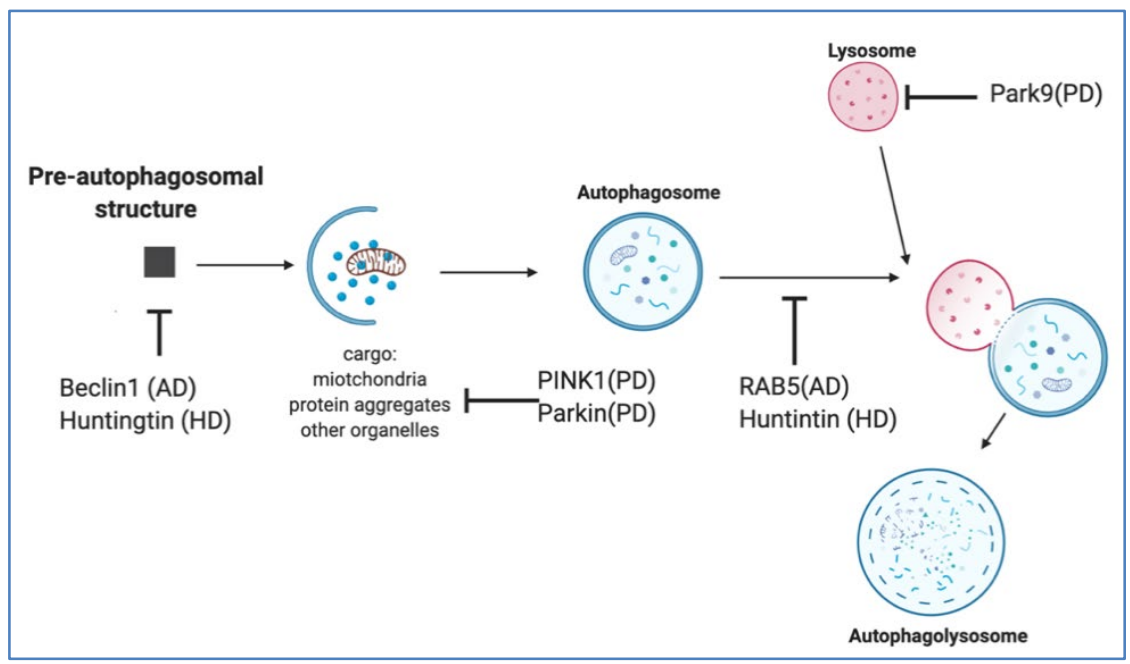

Figure B4. Autophagy mechanism and associated gene dysfunctions. Autophagy is a cellular mechanism by which metabolites, organelles, proteins and protein aggregates are enveloped by a vesicular membrane to form an autophagosome. This autophagosome is trafficked to a lysosome where fusion occurs, and lysosomal degradative enzymes break down the cargo. Dysfunction in several genes associated with neurodegenerative diseases have been implicated and are known to disrupt autophagy. Figure 4 image created using BioRender.com.

\section{Felodipine}

Felodipine is an antihypertensive drug originally approved by the FDA in 1988 [66]. It prevents hypertension by inhibiting L-type calcium channels and preventing calcium-dependent smooth muscle contractions, namely those responsible for constriction of the blood vessels [66] Currently, it is being investigated as an inducer of autophagy in Huntington's Disease and Parkinson's Disease models.

Calcium influx inhibition induces autophagy by reducing the activity of calciumdependent cysteine proteases, which are also known as calpains. Upon activation, calpains cleave G-proteins, which results in the activation of adenylyl cyclase. Activated adenylyl cyclase increases the amount of cyclic AMP (cAMP) in the cell and activates phospholipase C. Phospholipase C is responsible for the generation of phosphatidylinositol 3-phosphate (PIP3), which is a direct inhibitor of autophagy and 
allows more calcium to enter the cell creating a positive feedback loop. By blocking cytoplasmic calcium, there is a net reduction in PIP3 and an induction of autophagy [67].

Felodipine activity to induce autophagy by blocking L-type calcium channels was identified as favorable in a screen of drugs assessed in primary mice neurons [68] Out of nine calcium channel blockers, felodipine showed the greatest increase in autophagosomes and autophagolysosomes. The efficacy of felodipine has been further assessed in zebrafish models of tauopathy, a class of neurodegenerative disease involving the aggregation of Tau protein in the human brain, mouse models of Huntington's Disease, and mouse models of Parkinson's Disease [68]. In mice, felodipine has the ability to ameliorate signs of Huntington's disease over a treatment period of 12 weeks and resulted in improved clearance of alpha-synuclein, the main component of Lewy bodies. Twenty-two B6HD mice, a Huntington's Disease model, were treated with felodipine starting in their sixth week from birth, and were tested for grip strength, wire maneuvering and tremors, every two weeks from age 7 to 19 weeks. Felodipine treated mice were reported to have better grip strength from week 11-19 and a reduction in tremors from weeks 17-19, when compared to vehicle-treated control mice [68]. Likewise, A53T alpha-synuclein mice, a common Parkinson's Disease mouse model, were shown to have improved grip strength, increased neuron numbers in the substantia nigra (the area of the brain affected by Parkinson's Disease), and a reduction in insoluble alpha-synuclein, a protein that comprises Lewy bodies. In zebrafish, a rho: tau model was used to emulate cell death associated with the aggregation of Tau protein commonly associated with Alzheimer's Disease. In this model, wild-type human tau protein is expressed in rod photoreceptors and causes cell death. Felodipine successfully rescued 
rod cell death, but failed to do so in zebra fish lacking atg7, a vital autophagy gene, suggesting the effect of felodipine on these cells is dependent on autophagy [68].

While felodipine has shown promise as a potential new treatment for neurodegeneration, cautious skepticism is advised due to how recent the studies have been conducted and the scope of studies has been limited to preclinical models of disease. Human clinical trials and efficacious dosage regimens remain to be conducted. Due to differences between human and mouse physiology, felodipine is metabolized much quicker in mice. As a result, the mice in this study were exposed to transient exposures of felodipine that are two orders of magnitude higher than the dosage indicated for use as an antihypertensive drug in humans. It is possible that the required dosage is so high that deleterious side-effects may preclude human treatment. Finally, the models used in this work were only successful in showing that felodipine can clear protein aggregates; the studies did not include the full disease course or its progression.

\section{Lonafarnib}

Lonafarnib is a promising drug that has passed phase two clinical trials for the treatment of progeria, a genetic disorder causing accelerated aging in children, and phase three clinical trials for the treatment of hepatitis delta virus infections [69]. Lonafarnib is a farnesyltransferase inhibitor that prevents prenylation, the addition of a lipid group to the cysteine residues of proteins. In hepatitis delta infections, prenylation is required for the assembly and packaging of new viral particles. 68 By inhibiting prenylation, fewer viral particles are created. In progeria, a genetic mutation causes Lamin A to be truncated and form progerin. Lamin $\mathrm{A}$ is an important part of the nuclear membrane structure and 
may be required for the repair of double-stranded DNA breaks [70]. When progerin, is formed instead, it is prenylated and cannot incorporate into the nuclear membrane. By inhibiting farnesyltransferase and prenylation, the instability of the nuclear membrane can be rescued [70].

Interestingly, lonafarnib shows promise as an inducer of autophagy and may be a possible therapeutic to treat tauopathy. In the cell, autophagy is regulated by the mechanistic target of rapamycin (mTOR) kinase, also commonly referred to as FK506binding protein 12-rapamycin-associated protein 1 (FRAP1). When mTOR is active, autophagy is inhibited. mTOR itself is regulated by the G-protein, Rheb, which is functional when localized to the cell membrane via a fatty acid chain tether. Farnesyltransferase inhibitors, such as lonafarnib disrupt the prenylation of Rheb and prevent its localization to the inner cell membrane. Without Rheb in the proper position, mTOR cannot be activated, resulting in an upregulation of autophagy [71].

Lonafarnib has been shown to induce autophagy in primary mice fibroblast cells as well as improving symptoms in mice models of dementia. In a study by Hernandez et al., transgenic NIH3T3 mouse fibroblast cells were used to show efficacy in inducing autophagy [72]. NIH3T3 cells were used to perform a fluorescent assay that can determine the rate of autophagy[71]. NIH3T3 cells treated with increasing doses of lonafarnib exhibited dose-dependent increases in autophagolysosomes suggesting a lonafarnib mediated increase in autophagy. Furthermore, Tg4510 mice, used as a dementia model displaying behavioral impairments associated with dementia, were treated with lonafarnib starting at 10 weeks of age. At 20 weeks, mice were assessed for 
nest building behaviors. Treated mice displayed an improvement in nest shredding, a trait associated with these mice. Lonafarnib was also able to ameliorate the circling behavior attributed to this model, but not reverse it after already manifesting. Due to these findings, it was concluded that lonafarnib cannot reverse existing tau pathology, but can prevent it from worsening [72].

While lonafarnib is indeed a promising drug, it is important to consider that this drug is still in relatively early stages of the drug discovery pipeline. It has not yet been approved by the FDA and is not clinically available. It is also important to note that the successful use of this drug as a treatment for dementia would also be hindered by the fact that lonafarnib cannot reverse the damage done by tauopathy. Because of this, lonafarnib may be essential in patients with early diagnoses. Studies on the inhibition of prenylation of proteins should be performed to determine if lonafarnib may have any off-target effects, as there are many proteins that need to be prenylated to function correctly. It is likely that in the case of progeria, the life-saving effect seen by long term use of this drug outweighs any potentially harmful side-effects, but the same cannot be inferred for dementia.

\section{Inflammation and NRF2 as a Target in Neurodegeneration}

Chronic inflammation in the brain is associated with many neurodegenerative disorders including psoriasis, multiple sclerosis, Parkinson's disease, macular degeneration, cutaneous T-cell lymphoma, obstructive sleep apnea, adult brain glioblastoma and rheumatoid arthritis [64]. It is believed that the innate immune response and chronic inflammation of the brain mediated by microglia and astrocytes have a role 
in the onset of neurodegeneration, and there is interest in studying therapeutics to reduce neuroinflammation. As an example, nuclear factor (erythroid derived 2)-like2 (NRF2) is a transcription factor that regulates the expression of detoxifying enzymes and antioxidant genes including $\mathrm{NAD}(\mathrm{P}) \mathrm{H}$ quinone oxidoreductase 1, heme oxygenase1(HO-1), glutathione S-transferase (GST) genes, and UDP-glucuronosyltransferase (UGT) genes. NRF2 is inhibited by KEAP1 under normal conditions, but NRF2 is activated under stressful conditions. Under normal conditions, KEAP1 binds to NRF2 and mediates its degradation via the ubiquitin-proteasome system. However, under conditions of oxidative stress, KEAP1 releases NRF2 allowing it to upregulate any number of antioxidant genes. One such gene is HO-1. HO- 1 catalyzes the degradation of heme into carbon monoxide (CO), free iron, and biliverdin as well as directly upregulating anti-inflammatory cytokines and inhibiting pro-inflammatory cytokines. In addition to these points, $\mathrm{CO}$ inhibits $\mathrm{NF \kappa B}$, a signaling protein responsible for promoting pro-inflammatory cytokines [73]. Activation of the NRF2 signaling pathway, which is shown in (Figure B5), has a net anti-inflammatory effect. Dimethyl fumarate and exemestane are two drugs that could be repurposed for treatment of inflammation due to aberrant NRF2 signaling pathway. 


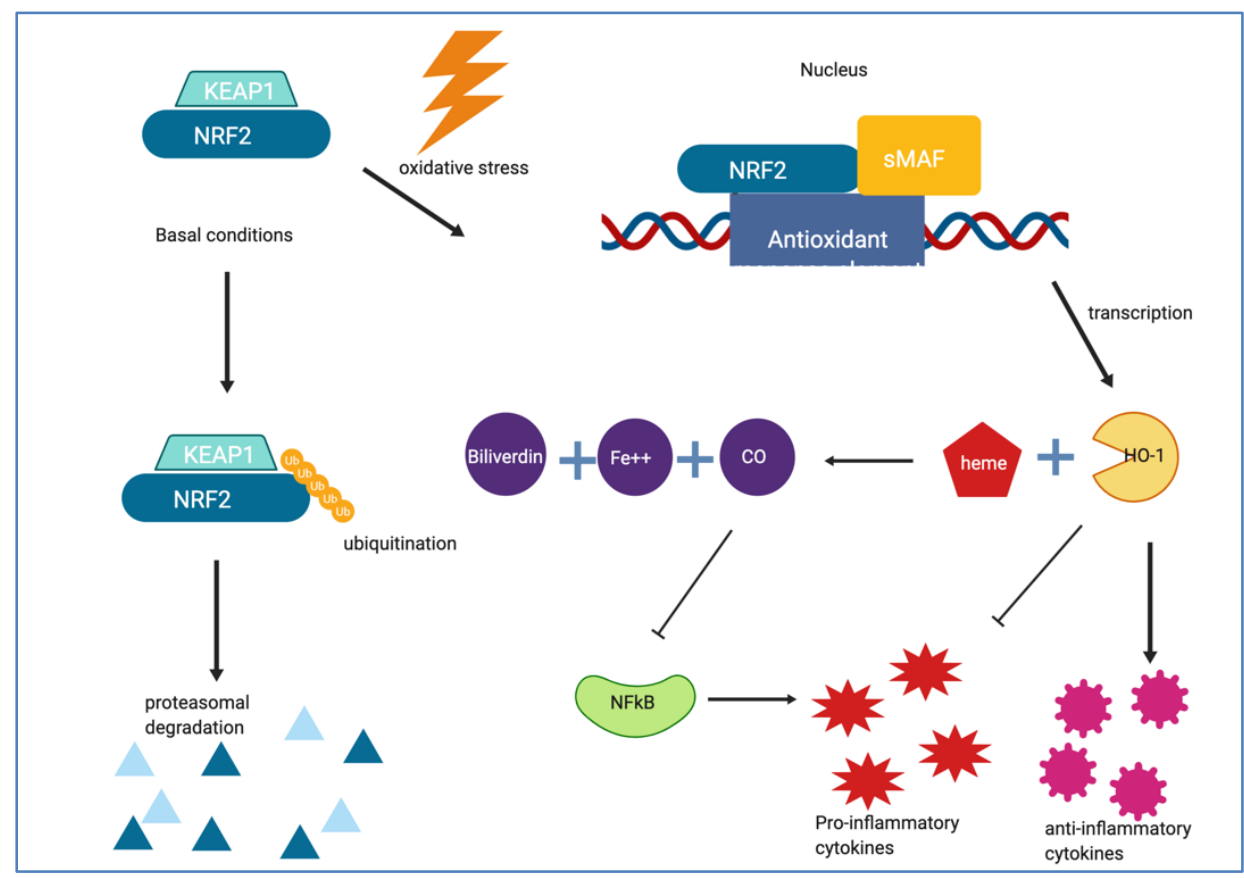

Figure B5. NRF2 signaling and inflammation. Under basal conditions, NRF2 is bound to its repressor, KEAP1 and ultimately degraded by the proteasome following ubiquination. However, under oxidative stress, free NRF2 translocates to the nucleus and dimerizes with small MAF family proteins. This complex binds to and promotes the expression of genes with an antioxidant response element, such as HO-1. HO-1 directly inhibits pro-inflammatory cytokines while upregulating antiinflammatory cytokines as well as catalyzing the breakdown of heme into carbon monoxide, free iron, and biliverdin. Carbon monoxide is an inhibitor of the NFkB pathway, resulting in an overall decrease of pro-inflammatory cytokines. The figure B5 image was created using BioRender.com.

\section{Dimethyl Fumarate}

Fumaric acid derivatives were first utilized in the 1950's for the treatment of psoriasis. The hypothesized mechanism of action for fumaric acid and its derivatives was thought to be that a combination of oral and topical administration of these compounds restored an imbalance in the citric acid cycle, as fumaric acid is an intermediate product in the cycle, and a fumarate deficiency was proposed as a problem in patients with psoriasis [74]. However, early clinical trials could not reproduce these results and fumarates were discontinued for over ten years. In the early 1990s, the first clinical trial for dimethyl fumarate (DMF) was conducted for the treatment of psoriasis and the results 
proved favorable in regards to severe plaque psoriasis [75]. The topical, exogenous administration of dimethyl fumarate (Figure B6), also known as Fumaderm®, evolved over time into an oral formulation in the late 1990 's. Germany licensed Fumaderm ${ }^{\circledR}$ as an oral treatment for moderate to severe psoriasis[76].

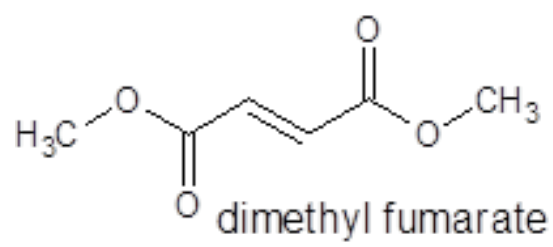

Figure B6. The structure for dimethyl fumarate.

DMF was approved by the FDA in 2014 under the trade name Tecfidera ${ }^{\circledR}$ as a medication to treat relapsing forms of multiple sclerosis (MS) [77,78]. Multiple sclerosis is an autoimmune disease in which the myelin sheath surrounding nerves is attacked and degrades, resulting in pain and motor function impairment. DMF is metabolized to monomethyl fumarate when administered to a patient. Monomethyl fumarate acts as an agonist of the nAChR, resulting in activation of the NRF2 pathway, which leads to a reduction in the inflammation that exacerbates demyelination. More recently, DMF has been found to activate this same anti-inflammatory pathway in Parkinson's disease patients, providing a promising treatment option for neurodegeneration that appears to work in mouse models of $\alpha$-synucleinopathy [79]. In PD, DMF has demonstrated a neuroprotective effect by ameliorating mitochondrial dysfunction and upregulating mitochondrial biogenesis by blocking neurotoxicity in wild-type, but not Nrf2 knockout mice [80].

On par with other immunomodulation treatments, DMF does not have a singular mechanism of action or one biochemical pathway that is targeted with administration. 
Rather, it is believed that DMF has multiple targets and exerts a spectrum of biological effect that leads to the therapeutic action of DMF experienced by MS patients. As shown in in vitro studies, DMF partially exerts anti-inflammatory action with the ability to increase the production of interleukin-4 (IL-4) and IL-5 when added to cultures of stimulated peripheral mononuclear blood cells. These cytokines are conducive to producing and promoting a Th2 immune response. The perturbation away from the Th1 response was also replicated in dendritic cells. The polarization of the immune response away from Th1 and toward the Th2 profile is a probable mechanism for DMF function as an immunomodulator. There is also evidence that DMF stimulates the native antioxidative stress machinery in cells. The anti-oxidative response is activated by NRF2, the primary transcription factor for genes associated with the anti-oxidative response. DMF has been shown to enhance the nuclear translocation of NRF2, and thus activating transcription of associated genes. The effect DMF has on the NRF2 pathway is likely a profound contributor to the mechanism of action [77].

In a study by Lastres-Becker et al., rAAV6-a-synuclein NRF2+/+ and NRF2-/mice were treated with DMF for 1 and 3 weeks followed by additional treatments every other day for eight weeks [79]. One day before sacrifice, the mice were assessed for motor asymmetry via the elevated body swing test. The NRF2-/- mice displayed significantly increased contralateral body torsion compared to the NRF2+/+ mice [81]. Additionally, DMF was assessed for its effect on signs of inflammation at the tissue level. One sign of inflammation in Parkinson's Disease is the increase of microglia and astrocytes in the basal ganglia known as microgliosis and astrocytosis, respectively. In order to test the effect of DMF on these inflammatory markers, NRF2 $+/+$ mice treated 
with DMF displayed significant decreases in astrocytosis and microgliosis compared to the NRF2-/- mice. In the brain, microglia can express one of two phenotypes, a proinflammatory, IL-1 $\beta$ and inducible nitric oxide synthase (iNOS)-producing phenotype and anti-inflammatory, IL-4 and sphingosine kinase 2-producing phenotype. DMF was shown to mediate the conversion of the pro-inflammatory phenotype to the antiinflammatory phenotype in mouse BV2 microglial cells. Cells treated with DMF displayed increased levels of IL-4 mRNA in a time-dependent manner [79]. DMF was also shown to have a neuroprotective effect on BV2 cells in studies where the cells were pretreated with DMF and then exposed to a-synuclein. Untreated cells had an induction of IL-1 $\beta$ and iNOS whereas pretreated cells displayed lower pro-inflammatory marker induction. Based on these findings, NRF2 appears to be a potentially useful target for Parkinson's Disease treatment, and DMF may bring us one step closer to a disease modifying drug [81].

A survey of clinical trial data yielded that DMF has been or is being investigated for efficacy in treating the following disease states: Primary Progressive Multiple Sclerosis, Relapsing-Remitting Multiple Sclerosis, Age-related Macular Degeneration, Cutaneous T Cell Lymphoma, Obstructive Sleep Apnea, Adult Brain Glioblastoma and Rheumatoid Arthritis. While there is a diversity of pathologies being investigated with the administration of DMF, inflammation arises as a shared theme in these clinical trials. Immunomodulation, down regulation of proinflammatory cytokines, the effect of DMF on $\mathrm{T}$ cell regulation, and DMF exerts in vitro could be of utility in treating a myriad of diseases [82-84]. 


\section{Exemestane}

Exemestane was approved by the FDA in 1999 and is marketed as Aromasin, an aromatase inhibitor for use on estrogen-dependent breast cancers, especially in postmenopausal women $[85,86]$. Exemestane treats breast cancer by preventing the synthesis of estrogen via the inhibition of the aromatization step of androgens into estradiol, a precursor of estrogen. Without estrogen, these estrogen-dependent cancers' growth and spread is inhibited. Exemestane was identified as a potential treatment for Parkinson's Disease through a screen aiming to find compounds that activate HO-1 [87]. Exemestane was found to be one of the most effective compounds in the screen. The proposed mechanism for treating Parkinson's Disease is different than that of treating breast cancer, where aromasin inhibits the formation of estrogen from androgen during the ratelimiting step of aromatase conversion [88,89]. In this sense, aromasin acts as an upregulator of NRF2 expression, a transcription factor responsible for producing antioxidant enzymes in order to circumvent the degeneration of dopaminergic neurons due to high amounts of oxidative stress from ROS such as NO [85,90].

In a study by Son et al, (2017), exemestane was validated in BV2 microglial cells and in MPTP mouse models of Parkinson's Disease. BV2 cells were tested for NRF2 protein levels, HO-1 and NQO1 mRNA levels, and iNOS and IL-1 $\beta$ levels following exemestane treatment.85 NRF2 expression was elevated, suggesting exemestanemediated activation. Likewise, the expression of NRF2 downstream genes increased with exemestane treatment, while inflammatory marker levels decreased. Taken together, these results signify a possible reduction of inflammation by exemestane. In the same study, exemestane was also evaluated in a mouse model of Parkinson's Disease. Mice 
were treated with MPTP, a neurotoxin that kills dopaminergic neurons and attempts to simulate the death of neurons in Parkinson's Disease. The mice were then coadministered with exemestane orally $(10 \mathrm{mg} / \mathrm{kg})$ three times a day every day for seven days. Following treatment, the mice were sacrificed. Immunostaining was performed for tyrosine hydroxylase, a selective marker expressed by dopaminergic neurons. Mice cotreated with exemestane exhibited a reduction in the loss of dopaminergic neurons caused by MPTP [85].

DMF and exemestane both show enticing results as potential new treatments for Parkinson's Disease; however, the exact molecular target of either drug remains unknown. The Parkinson's Disease model studies performed with DMF and exemestane are still preclinical, limiting any interpretation of results regarding potential efficacy in humans. One concern that may be raised for exemestane experiments has to do with the MPTP mouse model. The addition of a toxin does not necessarily recapitulate Parkinson's Disease progression because the neurons die all at once instead of gradually over time as they would in human disease progression. Additionally, the results from the Son et al study seem to contradict previous reports that estradiol has a neuroprotective effect in PD [16]. However, estradiol and its inhibitor, exemestane, exhibit their neuroprotective qualities through separate pathways- JNK and NRF2, respectively. This would necessitate further studies on the effects of estradiol and its inhibition in the context of PD. Another consideration that must be made is that the experiments conducted on mice for both drugs were done over a relatively short period of time. The DMF treatments lasted eight weeks and the exemestane tests were done for seven days. It will be necessary to discover how long-term treatments affect the progression of 
Parkinson's Disease in more relevant model systems up to and including human trials.

\section{Conclusion}

The benefit of repurposing drugs that have previously been FDA approved removes many of the time and financial barriers for bringing a drug to market. FDA approval is a good indication of a drug's effectiveness and safety in clinical trials, which is indicative of a good outcome for the use of a drug for a different purpose. Because a drug can take anywhere from 8-12 years to go from initial discovery to the market, drug repurposing can cut this time significantly[1]. The repurposing of drugs to treat cardiovascular and neurodegenerative diseases is an emerging and promising field of study. Treatments for inflammatory diseases including vascular endothelial dysfunction, pulmonary arterial hypertension, multiple sclerosis, Parkinson's, and Alzheimer's, using repurposed drugs like colchicine, methotrexate, tocilizumab, felodipine, lonafarnib, dimethyl fumarate and exemestane may prove to be an effective strategy to address the treatment gap for untreatable ailments 


\section{References}

(1) Scannell, J. W.; Blanckley, A.; Boldon, H.; Warrington, B. Diagnosing the Decline in Pharmaceutical R\&D Efficiency. Nat. Rev. Drug Discov. 2012, 11 (3), 191-200. https://doi.org/10.1038/nrd3681.

(2) GNS, H. S.; GR, S.; Murahari, M.; Krishnamurthy, M. An Update on Drug Repurposing: Re-Written Saga of the Drug's Fate. Biomed. Pharmacother. 2019, 110 (October 2018), 700-716. https://doi.org/10.1016/j.biopha.2018.11.127.

(3) Ashburn, T. T.; Thor, K. B. Drug Repositioning: Identifying and Developing New Uses for Existing Drugs. Nat. Rev. Drug Discov. 2004, 3 (8), 673-683. https://doi.org/10.1038/nrd1468.

(4) Hubsher, G.; Haider, M.; Okun, M. S. Amantadine: The Journey from Fighting Flu to Treating Parkinson Disease. Neurology 2012, 78 (14), 1096-1099. https://doi.org/10.1212/WNL.0b013e31824e8f0d.

(5) Smieszek, S. P.; Przychodzen, B. P.; Polymeropoulos, M. H. Amantadine Disrupts Lysosomal Gene Expression: A Hypothesis for COVID19 Treatment. Int. J. Antimicrob. Agents 2020, 55 (6), 106004. https://doi.org/10.1016/j.ijantimicag.2020.106004.

(6) Li, X.; Rousseau, J. F.; Ding, Y.; Song, M.; Lu, W. Understanding Drug Repurposing From the Perspective of Biomedical Entities and Their Evolution: Bibliographic Research Using Aspirin. JMIR Med. Informatics 2020, 8 (6), e16739. https://doi.org/10.2196/16739.

(7) Sanberg, P. R.; Vindrola-Padros, C.; Shytle, R. D. Translating Laboratory Discovery to the Clinic: From Nicotine and Mecamylamine to Tourette's, Depression, and Beyond. Physiol. Behav. 2012, 107 (5), 801-808. https://doi.org/10.1016/j.physbeh.2012.06.023.

(8) Varothai, S.; Bergfeld, W. F. Androgenetic Alopecia: An Evidence-Based Treatment Update. Am. J. Clin. Dermatol. 2014, 15 (3), 217-230. https://doi.org/10.1007/s40257-014-0077-5. 
(9) Miguel, D. C.; Yokoyama-Yasunaka, J. K. U.; Andreoli, W. K.; Mortara, R. A.; Uliana, S. R. B. Tamoxifen Is Effective against Leishmania and Induces a Rapid Alkalinization of Parasitophorous Vacuoles Harbouring Leishmania (Leishmania) Amazonensis Amastigotes. J. Antimicrob. Chemother. 2007, 60 (3), 526-534. https://doi.org/10.1093/jac/dkm219.

(10) Cao, C.; Moult, J. GWAS and Drug Targets. BMC Genomics 2014, 15 (Suppl 4), 1-14. https://doi.org/10.1186/1471-2164-15-S4-S5.

(11) Rudrapal, M.; J. Khairnar, S.; G. Jadhav, A. Drug Repurposing (DR): An Emerging Approach in Drug Discovery. In Drug Repurposing - Hypothesis, Molecular Aspects and Therapeutic Applications; IntechOpen, 2020; p 13. https://doi.org/10.5772/intechopen.93193.

(12) Campillos, M.; Kuhn, M.; Gavin, A. C.; Jensen, L. J.; Bork, P. Drug Target Identification Using Side-Effect Similarity. Science (80-. ). 2008, 321 (5886), 263266. https://doi.org/10.1126/science. 1158140.

(13) Tam, V.; Patel, N.; Turcotte, M.; Bossé, Y.; Paré, G.; Meyre, D. Benefits and Limitations of Genome-Wide Association Studies. Nat. Rev. Genet. 2019, 20 (8), 467-484. https://doi.org/10.1038/s41576-019-0127-1.

(14) Tam, V.; Patel, N.; Turcotte, M.; Bossé, Y.; Paré, G.; Meyre, D. Benefits and Limitations of Genome-Wide Association Studies. Nat. Rev. Genet. 2019, 20 (8), 467-484. https://doi.org/10.1038/s41576-019-0127-1.

(15) Miller, I. N.; Cronin-Golomb, A. Gender Differences in Parkinson's Disease: Clinical Characteristics and Cognition. Mov. Disord. 2010, 25 (16), 2695-2703. https://doi.org/10.1002/mds.23388.

(16) Sawada, H.; Ibi, M.; Kihara, T.; Honda, K.; Nakamizo, T.; Kanki, R.; Nakanishi, M.; Sakka, N.; Akaike, A.; Shimohama, S. Estradiol Protects Dopaminergic Neurons in a MPP+Parkinson's Disease Model. Neuropharmacology 2002, 42 (8), 1056-1064. https://doi.org/10.1016/S0028-3908(02)00049-7. 
(17) Yue, Z.; Arora, I.; Zhang, E. Y.; Laufer, V.; Bridges, S. L.; Chen, J. Y. Repositioning Drugs by Targeting Network Modules: A Parkinson's Disease Case Study. BMC Bioinformatics 2017, 18 (Suppl 14), 17-30. https://doi.org/10.1186/s12859-017-1889-0.

(18) Reilly, M. P.; Li, M.; He, J.; Ferguson, J. F.; Stylianou, I. M.; Mehta, N. N.; Burnett, M. S.; Devaney, J. M.; Knouff, C. W.; Thompson, J. R.; et al. Identification of ADAMTS7 as a Novel Locus for Coronary Atherosclerosis and Association of $\mathrm{ABO}$ with Myocardial Infarction in the Presence of Coronary Atherosclerosis: Two Genome-Wide Association Studies. Lancet 2011, 377 (9763), 383-392. https://doi.org/10.1016/S0140-6736(10)61996-4.

(19) Shu, L.; Blencowe, M.; Yang, X. Translating GWAS Findings to Novel Therapeutic Targets for Coronary Artery Disease. Front. Cardiovasc. Med. 2018, 5 (May), 1-9. https://doi.org/10.3389/fcvm.2018.00056.

(20) King, M. D.; Long, T.; Pfalmer, D.; Andersen, T.; Mcdougal, O. M. SPIDR: Small-Molecule Peptide-Influenced Drug Repurposing.

(21) Bullock, C.; Cornia, N.; Jacob, R.; Remm, A.; Peavey, T.; Weekes, K.; Mallory, C.; Oxford, J. T.; McDougal, O. M.; Andersen, T. L. DockoMatic 2.0: High Throughput Inverse Virtual Screening and Homology Modeling. J. Chem. Inf. Model. 2013, 53 (8), 2161-2170. https://doi.org/10.1021/ci400047w.

(22) Marquart, L. A.; Turner, M. W.; McDougal, O. M. Qualitative Assay to Detect Dopamine Release by Ligand Action on Nicotinic Acetylcholine Receptors. Toxins (Basel). 2019, 11 (12), 1-14. https://doi.org/10.3390/toxins11120682.

(23) Trott, O.; Olson, A. Autodock Vina: Improving the Speed and Accuracy of Docking. J. Comput. Chem. 2010, 31 (2), 455-461. https://doi.org/10.1002/jcc.21334.AutoDock.

(24) Durães, F.; Pinto, M.; Sousa, E. Old Drugs as New Treatments for Neurodegenerative Diseases. Pharmaceuticals 2018, 11 (2), 1-21. https://doi.org/10.3390/ph11020044. 
(25) Mangoni, A. A.; Tommasi, S.; Zinellu, A.; Sotgia, S.; Carru, C.; Piga, M.; Erre, G. L. Repurposing Existing Drugs for Cardiovascular Risk Management: A Focus on Methotrexate. Drugs Context 2018, 7, 1-12. https://doi.org/10.7573/dic.212557.

(26) Murphy, S. L.; Xu, J.; Kochanek, K. D.; Arias, E.; Ph, D. Mortality in the United States , 2017; 2018.

(27) Pinkaew, D.; Fujise, K. Fortilin: A Potential Target for the Prevention and Treatment of Human Diseases. Adv. Clin. Chem. 2017, 82, 265-300. https://doi.org/10.1016/bs.acc.2017.06.006.

(28) Feletou, M. The Endothelium: Part 1: Multiple Functions Ofthe Endothelial Cells-Focus on Endothelium-Derived Vasoactive Mediators; Morgan \& Claypool Life Sciences: San Rafael, 2011.

(29) Sakurada, M.; Yoshioka, N.; Kuse, A.; Nakagawa, K.; Morichika, M.; Takahashi, M.; Kondo, T.; Asano, M.; Ueno, Y. Rapid Identification of Gloriosa Superba and Colchicum Autumnale by Melting Curve Analysis: Application to a Suicide Case Involving Massive Ingestion of G. Superba. Int. J. Legal Med. 2019, 133 (4), 1065-1073. https://doi.org/10.1007/s00414-019-02060-х.

(30) Ross, R. Atherosclerosis -- an Inflammatory Disease. N. Engl. J. Med. 1999, 340 (2), 115-126.

(31) Libby, P.; Hansson, G. K. Inflammation and Immunity in Diseases of the Arterial Tree: Players and Layers. Circ. Res. 2015, 116 (2), 307-311. https://doi.org/10.1161/CIRCRESAHA.116.301313.

(32) Davies, M. J. Stability and Instability: Two Faces of Coronary Atherosclerosis: The Paul Dudley White Lecture 1995. Circulation 1996, 94 (8), 2013-2020. https://doi.org/10.1161/01.CIR.94.8.2013.

(33) Verma, S.; Szmitko, P. E.; Ridker, P. M. C-Reactive Protein Comes of Age. Nat. Clin. Pract. Cardiovasc. Med. 2005, 2 (1), 29-36. https://doi.org/10.1038/ncpcardio0074. 
(34) Nidorf, S. M.; Thompson, P. L. Why Colchicine Should Be Considered for Secondary Prevention of Atherosclerosis: An Overview. Clin. Ther. 2019, 41 (1), 41-48. https://doi.org/10.1016/j.clinthera.2018.11.016.

(35) Fiolet, A. T. L.; Nidorf, S. M.; Mosterd, A.; Cornel, J. H. Colchicine in Stable Coronary Artery Disease. Clin. Ther. 2019, 41 (1), 30-40. https://doi.org/10.1016/j.clinthera.2018.09.011.

(36) Leung, Y. Y.; Yao Hui, L. L.; Kraus, V. B. Colchicine-Update on Mechanisms of Action and Therapeutic Uses. Semin. Arthritis Rheum. 2015, 45 (3), 341-350. https://doi.org/10.1016/j.semarthrit.2015.06.013.

(37) Redelinghuys, P.; Brown, G. D. Inhibitory C-Type Lectin Receptors in Myeloid Cells. Immunol. Lett. 2011, $136(1), 1-12$. https://doi.org/10.1016/j.imlet.2010.10.005.

(38) Brennan, K.; Zheng, J. Interleukin 8. In xPharm: The Comprehensive Pharmacology Reference; Elsevier, 2007; pp 1-4. https://doi.org/10.1016/B978008055232-3.61916-6.

(39) Smith, A.; Hum, M.; Winick, N. J.; Kamen, B. A. A Case for the Use of Aminopterin in Treatment of Patients with Leukemia Based on Metabolic Studies of Blasts in Vitro. Clin. Cancer Res. 1996, 2 (1), 69-73.

(40) Weinblatt, M. E. Methotrexate in Rheumatoid Arthritis: A Quarter Century of Development. Trans. Am. Clin. Climatol. Assoc. 2013, 124, 16-25.

(41) Kinder, A. J.; Hassell, A. B.; Brand, J.; Brownfield, A.; Grove, M.; Shadforth, M. F. The Treatment of Inflammatory Arthritis with Methotrexate in Clinical Practice: Treatment Duration and Incidence of Adverse Drug Reactions. Rheumatology 2005, 44 (1), 61-66. https://doi.org/10.1093/rheumatology/keh512.

(42) Cronstein, B. N.; Aune, T. M. Methotrexate and Its Mechanisms of Action in Inflammatory Arthritis. Nat. Rev. Rheumatol. 2020, 16 (3), 145-154. https://doi.org/10.1038/s41584-020-0373-9. 
(43) Sankrityayan, H.; Majumdar, A. S. Curcumin and Folic Acid Abrogated Methotrexate Induced Vascular Endothelial Dysfunction. Can. J. Physiol.

Pharmacol. 2015, 94 (1), 89-96. https://doi.org/10.1139/cjpp-2015-0156.

(44) Panja, S.; Khatua, D. K.; Halder, M. Simultaneous Binding of Folic Acid and Methotrexate to Human Serum Albumin: Insights into the Structural Changes of Protein and the Location and Competitive Displacement of Drugs. ACS Omega 2018, 3 (1), 246-253. https://doi.org/10.1021/acsomega.7b01437.

(45) Crabtree, M. J.; Hale, A. B.; Channon, K. M. Dihydrofolate Reductase Protects Endothelial Nitric Oxide Synthase from Uncoupling in Tetrahydrobiopterin Deficiency. Free Radic. Biol. Med. 2011, 50 (11), 1639-1646. https://doi.org/10.1016/j.freeradbiomed.2011.03.010.

(46) Zeng, L.; Yan, Z.; Ding, S.; Xu, K.; Wang, L. Endothelial Injury, an Intriguing Effect of Methotrexate and Cyclophosphamide During Hematopoietic Stem Cell Transplantation in Mice. Transplant. Proc. 2008, 40 (8), 2670-2673. https://doi.org/10.1016/j.transproceed.2008.06.038.

(47) Matei, V.; Rodríguez-Vilarrupla, A.; Deulofeu, R.; Colomer, D.; Fernández, M.; Bosch, J.; Garcia-Pagán, J. C. The ENOS Cofactor Tetrahydrobiopterin Improves Endothelial Dysfunction in Livers of Rats with CCl4 Cirrhosis. Hepatology 2006, 44 (1), 44-52. https://doi.org/10.1002/hep.21228.

(48) Gualtierotti, R.; Ingegnoli, F.; Boscolo, M.; Griffini, S.; Grovetti, E.; Cugno, M. Tocilizumab Effects on Coagulation Factor XIII in Patients with Rheumatoid Arthritis. Adv. Ther. 2019. https://doi.org/10.1007/s12325-019-01118-x.

(49) Ruiz-Limón, P.; Ortega, R.; Arias de la Rosa, I.; Abalos-Aguilera, M. del C.; Perez- Sanchez, C.; Jimenez- Gomez, Y.; Peralbo-Santaella, E.; Font, P.; RuizVilches, D.; Ferrin, G.; et al. Tocilizumab Improves the Proatherothrombotic Profile of Rheumatoid Arthritis Patients Modulating Endothelial Dysfunction, NETosis, and Inflammation. Transl. Res. 2017, 183, 87-103. https://doi.org/10.1016/j.trsl.2016.12.003. 
(50) Del Rincón, I.; Williams, K.; Stern, M. P.; Freeman, G. L.; Escalante, A. High Incidence of Cardiovascular Events in a Rheumatoid Arthritis Cohort Not Explained by Traditional Cardiac Risk Factors. Arthritis Rheum. 2001, 44 (12), 2737-2745. https://doi.org/10.1002/1529-0131(200112)44:12<2737::aid$\operatorname{art} 460>3.0 . c 0 ; 2-\% 23$.

(51) Hoeper, M. M.; Gibbs, J. S. R. The Changing Landscape of Pulmonary Arterial Hypertension and Implications for Patient Care. Eur. Respir. Rev. 2014, 23 (134), 450-457. https://doi.org/10.1183/09059180.00007814.

(52) Gaine, S. P.; Rubin, L. J. Primary Pulmonary Hypertension. Lancet 1998, 352, $719-725$.

(53) Sitbon, O.; Vonk Noordegraaf, A. Epoprostenol and Pulmonary Arterial Hypertension: 20 Years of Clinical Experience. Eur. Respir. Rev. 2017, 26 (143). https://doi.org/10.1183/16000617.0055-2016.

(54) Leeper, B.; Powell, B. Pulmonary Arterial Hypertension. Nurs. Crit. Care 2019, 14 (3), 14-22. https://doi.org/10.1097/01.CCN.0000554829.05209.ca.

(55) Grinnan, D.; Trankle, C.; Andruska, A.; Bloom, B.; Spiekerkoetter, E. Drug Repositioning in Pulmonary Arterial Hypertension: Challenges and Opportunities. Pulm. Circ. 2019, 9 (1). https://doi.org/10.1177/2045894019832226.

(56) Trankle, C. R.; Canada, J. M.; Kadariya, D.; Markley, R.; De Chazal, H. M.; Pinson, J.; Fox, A.; Van Tassell, B. W.; Abbate, A.; Grinnan, D. IL-1 Blockade Reduces Inflammation in Pulmonary Arterial Hypertension and Right Ventricular Failure: A Single-Arm, Open-Label, Phase Ib/II Pilot Study. Am. J. Respir. Crit. Care Med. 2019, 199 (3), 381-384. https://doi.org/10.1164/rccm.201809-1631LE.

(57) Parpaleix, A.; Amsellem, V.; Houssaini, A.; Abid, S.; Breau, M.; Marcos, E.; Sawaki, D.; Delcroix, M.; Quarck, R.; Maillard, A.; et al. Role of Interleukin-1 Receptor 1/MyD88 Signalling in the Development and Progression of Pulmonary Hypertension. Eur. Respir. J. 2016, 48 (2), 470-483. https://doi.org/10.1183/13993003.01448-2015. 
(58) Ota, K.; Uzuka, Y. Clinical Trials of Bestatin for Leukemia and Solid Tumors. Biotherapy 1992, 4 (3), 205-214.

(59) Newman, J. W.; Morisseau, C.; Hammock, B. D. Epoxide Hydrolases: Their Roles and Interactions with Lipid Metabolism. Prog. Lipid Res. 2005, 44 (1), 1-51. https://doi.org/10.1016/j.plipres.2004.10.001.

(60) Bredesen, D. E.; Rao, R. V.; Mehlen, P. Cell Death in the Nervous System. Nature 2006, 443 (7113), 796-802. https://doi.org/10.1038/nature05293.

(61) Rubinsztein, D. C. The Roles of Intracellular Protein-Degradation Pathways in Neurodegeneration. Nature 2006, 443 (7113), 780-786. https://doi.org/10.1038/nature05291.

(62) Chen, X.; Pan, W. The Treatment Strategies for Neurodegenerative Diseases by Integrative Medicine. Integr. Med. Int. 2015, 1 (4), 223-225. https://doi.org/10.1159/000381546.

(63) Mizushima, N.; Komatsu, M. Autophagy: Renovation of Cells and Tissues. Cell 2011, 147 (4), 728-741. https://doi.org/10.1016/j.cell.2011.10.026.

(64) Amor, S.; Puentes, F.; Baker, D.; Van Der Valk, P. Inflammation in Neurodegenerative Diseases. Immunology 2010, 129 (2), 154-169. https://doi.org/10.1111/j.1365-2567.2009.03225.x.

(65) Martinez-Vicente, M. Autophagy in Neurodegenerative Diseases: From Pathogenic Dysfunction to Therapeutic Modulation. Semin. Cell Dev. Biol. 2015, 40, 115-126. https://doi.org/10.1016/j.semcdb.2015.03.005.

(66) Plendil Drug Approval Package.

(67) Tian, X.; Gala, U.; Zhang, Y.; Shang, W.; Nagarkar Jaiswal, S.; di Ronza, A.; Jaiswal, M.; Yamamoto, S.; Sandoval, H.; Duraine, L.; et al. A Voltage-Gated Calcium Channel Regulates Lysosomal Fusion with Endosomes and Autophagosomes and Is Required for Neuronal Homeostasis. PLoS Biol. 2015, 13 (3), 1-25. https://doi.org/10.1371/journal.pbio.1002103. 
(68) Siddiqi, F. H.; Menzies, F. M.; Lopez, A.; Stamatakou, E.; Karabiyik, C.; Ureshino, R.; Ricketts, T.; Jimenez-Sanchez, M.; Esteban, M. A.; Lai, L.; et al. Felodipine Induces Autophagy in Mouse Brains with Pharmacokinetics Amenable to Repurposing. Nat. Commun. 2019, 10 (1), 1-14. https://doi.org/10.1038/s41467019-09494-2.

(69) Alto, P.; Biopharmaceuticals, E. Eiger Announces Breakthrough Therapy Designation Granted by FDA for Lonafarnib in Progeria and Progeroid Laminopathies Survival Benefit in Children with Progeria Published in JAMA 2018 NDA Filing Planned in 2019. 2019.

(70) Sinha, J. K.; Ghosh, S.; Raghunath, M. Progeria: A Rare Genetic Premature Ageing Disorder. Indian J. Med. Res. 2014, 139 (MAY), 667-674.

(71) Pan, J.; Song, E.; Cheng, C.; Lee, M. H.; Yeung, S. C. J. Farnesyltransferase Inhibitors-Induced Autophagy: Alternative Mechanisms? Autophagy 2009, 5 (1), 129-131. https://doi.org/10.4161/auto.5.1.7329.

(72) Hernandez, I.; Luna, G.; Rauch, J. N.; Reis, S. A.; Giroux, M.; Karch, C. M.; Boctor, D.; Sibih, Y. E.; Storm, N. J.; Diaz, A.; et al. A Farnesyltransferase Inhibitor Activates Lysosomes and Reduces Tau Pathology in Mice with Tauopathy. Sci. Transl. Med. 2019, 11 (485), eaat3005. https://doi.org/10.1126/scitranslmed.aat3005.

(73) Ahmed, S. M. U.; Luo, L.; Namani, A.; Wang, X. J.; Tang, X. Nrf2 Signaling Pathway: Pivotal Roles in Inflammation. Biochim. Biophys. Acta-Mol. Basis Dis. 2017, 1863 (2), 585-597. https://doi.org/10.1016/j.bbadis.2016.11.005.

(74) D.M.W. Balak, D. Fumaric Acid Esters in the Management of Psoriasis. Psoriasis Targets Ther. 2015, 9. https://doi.org/10.2147/PTT.S51490.

(75) D.M.W. Balak, D. Fumaric Acid Esters in the Management of Psoriasis. Psoriasis Targets Ther. 2015, 9. https://doi.org/10.2147/PTT.S51490.

(76) Linker, R. A.; Haghikia, A. Dimethyl Fumarate in Multiple Sclerosis: Latest Developments, Evidence and Place in Therapy. Ther. Adv. Chronic Dis. 2016, 7 (4), 198-207. https://doi.org/10.1177/2040622316653307. 
(77) Bomprezzi, R. Dimethyl Fumarate in the Treatment of Relapsing-Remitting Multiple Sclerosis: An Overview. Ther. Adv. Neurol. Disord. 2015, 8 (1), 20-30. https://doi.org/10.1177/1756285614564152.

(78) Tecfidera Drug Approval Package https:/www.accessdata.fda.gov/drugsatfda_docs/nda/2013/204063Orig1s000TOC. cfm (accessed Dec 10, 2020).

(79) Lastres-Becker, I.; García-Yagüe, A. J.; Scannevin, R. H.; Casarejos, M. J.; Kügler, S.; Rábano, A.; Cuadrado, A. Repurposing the NRF2 Activator Dimethyl Fumarate as Therapy Against Synucleinopathy in Parkinson's Disease.

Antioxidants Redox Signal. 2016, 25 (2), 61-77.

https://doi.org/10.1089/ars.2015.6549.

(80) Bento-Pereira, C.; Dinkova-Kostova, A. T. Activation of Transcription Factor Nrf2 to Counteract Mitochondrial Dysfunction in Parkinson's Disease. Med. Res. Rev. 2021, 41 (2), 785-802. https://doi.org/10.1002/med.21714.

(81) Aromasin Drug Approval Package.

(82) Braley, T. J.; Huber, A. K.; Segal, B. M.; Kaplish, N.; Saban, R.; WashnockSchmid, J. M.; Chervin, R. D. A Randomized, Subject and Rater-Blinded, PlaceboControlled Trial of Dimethyl Fumarate for Obstructive Sleep Apnea. Sleep 2018, 41 (8), 1-10. https://doi.org/10.1093/sleep/zsy109.

(83) Shafer, D.; Tombes, M. B.; Shrader, E.; Ryan, A.; Bandyopadhyay, D.; Dent, P.; Malkin, M. Phase I Trial of Dimethyl Fumarate, Temozolomide, and Radiation Therapy in Glioblastoma. Neuro-Oncology Adv. 2020, 2 (1), 1-5. https://doi.org/10.1093/noajnl/vdz052.

(84) Schlöder, J.; Berges, C.; Luessi, F.; Jonuleit, H. Dimethyl Fumarate Therapy Significantly Improves the Responsiveness of T Cells in Multiple Sclerosis Patients for Immunoregulation by Regulatory T Cells. Int. J. Mol. Sci. 2017, 18 (2). https://doi.org/10.3390/ijms 18020271. 
(85) Son, H. J.; Han, S. H.; Lee, J. A.; Shin, E. J.; Hwang, O. Potential Repositioning of Exemestane as a Neuroprotective Agent for Parkinson's Disease. Free Radic. Res. 2017, 51 (6), 633-645. https://doi.org/10.1080/10715762.2017.1353688.

(86) Yamamoto, Y.; Ishikawa, T.; Hozumi, Y.; Ikeda, M.; Iwata, H.; Yamashita, H.; Toyama, T.; Chishima, T.; Saji, S.; Yamamoto-Ibusuki, M.; et al. Randomized Controlled Trial of Toremifene $120 \mathrm{Mg}$ Compared with Exemestane $25 \mathrm{Mg}$ after Prior Treatment with a Non-Steroidal Aromatase Inhibitor in Postmenopausal Women with Hormone Receptor-Positive Metastatic Breast Cancer. BMC Cancer 2013, 13, 1-9. https://doi.org/10.1186/1471-2407-13-239.

(87) Woo, S. Y.; Kim, J. H.; Moon, M. K.; Han, S. H.; Yeon, S. K.; Choi, J. W.; Jang, B. K.; Song, H. J.; Kang, Y. G.; Kim, J. W.; et al. Discovery of Vinyl Sulfones as a Novel Class of Neuroprotective Agents toward Parkinson's Disease Therapy. J. Med. Chem. 2014, 57 (4), 1473-1487. https://doi.org/10.1021/jm401788m.

(88) Liu, H.; Talalay, P. Relevance of Anti-Inflammatory and Antioxidant Activities of Exemestane and Synergism with Sulforaphane for Disease Prevention. Proc. Natl. Acad. Sci. U. S. A. 2013, 110 (47), 19065-19070. https://doi.org/10.1073/pnas.1318247110.

(89) Miller, W. R.; Bartlett, J.; Brodie, A. M. H.; Brueggemeier, R. W.; di Salle, E.; Lønning, P. E.; Llombart, A.; Maass, N.; Maudelonde, T.; Sasano, H.; et al. Aromatase Inhibitors: Are There Differences Between Steroidal and Nonsteroidal Aromatase Inhibitors and Do They Matter? Oncologist 2008, 13 (8), 829-837. https://doi.org/10.1634/theoncologist.2008-0055.

(90) Sandberg, M.; Patil, J.; D’Angelo, B.; Weber, S. G.; Mallard, C. NRF2-Regulation in Brain Health and Disease: Implication of Cerebral Inflammation.

Neuropharmacology 2014, 79, 298-306.

https://doi.org/10.1016/j.neuropharm.2013.11.004. 